\title{
Determining the true mass of radial-velocity exoplanets with Gaia
}

\section{Nine planet candidates in the brown dwarf or stellar regime and 27 confirmed planets $\star$}

\author{
F. Kiefer ${ }^{1,2}$, G. Hébrard ${ }^{1,3}$, A. Lecavelier des Etangs ${ }^{1}$, E. Martioli ${ }^{1,4}$, S. Dalal $^{1}$, and A. Vidal-Madjar ${ }^{1}$ \\ ${ }^{1}$ Institut d'Astrophysique de Paris, Sorbonne Université, CNRS, UMR 7095, 98 bis bd Arago, 75014 Paris, France \\ e-mail: kiefer@iap.fr \\ ${ }^{2}$ LESIA, Observatoire de Paris, Université PSL, CNRS, Sorbonne Université, Université de Paris, 5 place Jules Janssen, \\ 92195 Meudon, France \\ ${ }^{3}$ Observatoire de Haute-Provence, CNRS, Université d'Aix-Marseille, 04870 Saint-Michel-1'Observatoire, France \\ ${ }^{4}$ Laboratório Nacional de Astrofísica, Rua Estados Unidos 154, 37504-364, Itajubá - MG, Brazil
}

Received 12 August 2020 / Accepted 24 September 2020

\begin{abstract}
Mass is one of the most important parameters for determining the true nature of an astronomical object. Yet, many published exoplanets lack a measurement of their true mass, in particular those detected as a result of radial-velocity (RV) variations of their host star. For those examples, only the minimum mass, or $m \sin i$, is known, owing to the insensitivity of RVs to the inclination of the detected orbit compared to the plane of the sky. The mass that is given in databases is generally that of an assumed edge-on system $\left(\sim 90^{\circ}\right)$, but many other inclinations are possible, even extreme values closer to $0^{\circ}$ (face-on). In such a case, the mass of the published object could be strongly underestimated by up to two orders of magnitude. In the present study, we use GASTON, a recently developed tool taking advantage of the voluminous Gaia astrometric database to constrain the inclination and true mass of several hundreds of published exoplanet candidates. We find nine exoplanet candidates in the stellar or brown dwarf (BD) domain, among which six were never characterized. We show that 30 Ari B b, HD 141937 b, HD 148427 b, HD 6718 b, HIP 65891 b, and HD 16760 b have masses larger than $13.5 M_{\mathrm{J}}$ at $3 \sigma$. We also confirm the planetary nature of 27 exoplanets, including HD $10180 \mathrm{c}, \mathrm{d}$ and g. Studying the orbital periods, eccentricities, and host-star metallicities in the BD domain, we found distributions with respect to true masses consistent with other publications. The distribution of orbital periods shows of a void of BD detections below $\sim 100 \mathrm{~d}$, while eccentricity and metallicity distributions agree with a transition between BDs similar to planets and BDs similar to stars in the range $40-50 M_{\mathrm{J}}$.
\end{abstract}

Key words. planets and satellites: fundamental parameters - brown dwarfs - binaries: general - astrometry

\section{Introduction}

A large fraction of exoplanets published in all up-to-date catalogs, such as www. exoplanet.eu (Schneider et al. 2011) or the NASA exoplanet archive (Akeson et al. 2013), have been detected as a consequence of radial-velocity (RV) variations of their host star. If the minimum mass $m \sin i$, where $i$ is a common symbolic notation for "orbital inclination", is located below the planet/brown dwarf (BD) critical mass of $13.5 M_{\mathrm{J}}$ such detection has to be considered as a new "candidate" planet. If any observed system is inclined according to an isotropic distribution, there is a nonzero probability $1-\cos I_{\mathrm{c}}$, where $I_{\mathrm{c}}$ is the inclination of the candidate orbit, that the $m \sin i$ underestimates the true mass of the companion by a factor larger than $1 / \sin I_{\mathrm{c}}$. Assuming $I_{\mathrm{c}}=10^{\circ}$, this already leads to a factor $\sim 6$, with a probability of $1.5 \%$. Such a small rate, considering the 500-1000 planets detected through RVs, implies that the mass is highly underestimated in only a few tens of planets. However, exoplanets catalogs usually neglect an important number of companions whose $m \sin i$ is larger than $13.5 M_{\mathrm{J}}$. The RV-detected samples of exoplanets in catalogs are partly biased toward small

\footnotetext{
* Full Tables 2, 3, 5, and 9 are only available at the CDS via anonymous ftp to cdsarc.u-strasbg.fr $(130.79 .128 .5)$ or via http://cdsarc.u-strasbg.fr/viz-bin/cat/J/A+A/645/A7
}

$m \sin i$ candidates and thus toward small inclinations (see, e.g. Han et al. 2001 for a related discussion). These samples likely contain more than a few tens of objects whose actual mass is larger than $13.5 \mathrm{MJ}_{\mathrm{J}}$.

Recovering the exact mass ratio distribution of binary companions from their mass function, therefore bypassing the issue of unknown inclination by using inversion techniques, such as the Lucy-Richardson algorithm (Richardson 1972; Lucy 1974), is a famous well-studied problem (Mazeh \& Goldberg 1992; Heacox 1995; Shahaf et al. 2017). Such inversion algorithms were applied to RV exoplanets mass distribution (Zucker \& Mazeh 2001a; Jorissen et al. 2001; Tabachnik \& Tremaine 2002). However, it is a statistical problem that cannot determine individual masses. This algorithm also lacks strong validation by comparison to exact mass distributions in the stellar or planetary regime. Moreover, the distribution of binary companions in the BD to M dwarf domain, with orbital periods of $1-10^{4} \mathrm{~d}$, from which exoplanet candidates could be originating, is not well described. Detections are still lacking in the BD regime - the socalled BD desert (Marcy \& Butler 2000) - although this region is constantly being populated (Halbwachs et al. 2000; Sahlmann et al. 2011a; Díaz et al. 2013; Ranc et al. 2015; Wilson et al. 2016; Kiefer et al. 2019). Our sparse knowledge of the low-mass tail of the population of stellar binary companions does not allow us 
Table 1. Comparing the three main databases with respect to the five criteria given in the text.

\begin{tabular}{|c|c|c|c|c|}
\hline Criterion & wWW.exoplanet.eu & NASA & Exoplanet Archive & www. exoplanets.org \\
\hline (0) None & 4302 & & 4197 & 3262 \\
\hline (1) $K, P, e, M_{\star}$ exist & 752 & & 1237 & 967 \\
\hline (2) $K>0, P>0, e \geq 0, M_{\star}>0$ & 750 & & 1237 & 961 \\
\hline (3) $+T_{\mathrm{p}}$ and $\omega$ exist if $e \geq 0.1$ & 582 & & 909 & 924 \\
\hline (4) + measured $m \sin i$ & 360 & & 580 & 911 \\
\hline (5) Last update considered & $10 / 08 / 2020$ & & 23/07/2020 & June 2018 \\
\hline
\end{tabular}

Notes. The five first lines provide the number of exoplanets.

to disentangle low sin $i$ BD or M-dwarf from real exoplanets. It also motivates us to extensively characterize the orbital inclination and true mass of companions in the exoplanet to M-dwarf regime.

The true mass of an individual RV exoplanet candidate can be determined by directly measuring the inclination angle of its orbit compared to the plane of the sky. If the companion is on an edge-on orbit $\left(I_{\mathrm{c}} \sim 90^{\circ}\right)$, then it is likely transiting and could be detected using photometric monitoring. Commonly, the transiting exoplanets are detected first with photometry and then characterized in mass with RV. About half of the exoplanets observed in RV were detected by transit photometry. The other half are not known to transit and the main options to measure the inclination of an exoplanet orbit are mutual interactions in the case of multiple planets systems and astrometry.

Astrometry has been used to determine the mass of exoplanet candidates in many studies. Observations with the Hubble Space Telescope Fine-Guidance-Sensor (HST/FGS) led to the confirmation of a few planets, in particular GJ 876 b (Benedict et al. 2002), and $\epsilon$-Eri b (Benedict et al. 2006). It also led to the corrected mass of planet candidates beyond the deuterium-burning limit; these include HD 38529 b, with a mass in the BD regime of $17 M_{\mathrm{J}}$ (Benedict et al. 2010); and HD 33636 b, with an M-dwarf mass of $140 M_{\mathrm{J}}$ (Bean et al. 2007). HiPPARCOS data were also extensively used to that purpose (Perryman et al. 1996; Mazeh et al. 1999; Zucker \& Mazeh 2001a; Sozzetti \& Desidera 2010; Sahlmann et al. 2011a; Reffert \& Quirrenbach 2011; Díaz et al. 2012; Wilson et al. 2016; Kiefer et al. 2019) but only yielded masses in the BD to M-dwarf regime. More recently, Gaia astrometric data were used for the first time to determine the mass of RV exoplanet candidates; various methods were used, for example, based on astrometric excess noise for HD $114762 \mathrm{~b}$, showing this object is stellar in nature (Kiefer 2019) and by comparing Gaia proper motion to HIPPARCOS proper motion in the case of Proxima b, confirming its planetary nature (Kervella et al. 2020). It is expected that, by the end of its mission, Gaia will provide the most precise and voluminous astrometry able to characterize exoplanet companions and even detect new exoplanets (Perryman et al. 2014).

In the present study, we aim to assess the nature of numerous RV-detected exoplanet candidates publicly available in exoplanets catalogs using the astrometric excess noise from the first data release (DR1) of the Gaia mission (Gaia Collaboration 2016). We use the recently developed method called Gaia Astrometric noise Simulation To derive Orbit iNclination (GASTON; Kiefer et al. 2019; Kiefer 2019) to constrain, from the astrometric excess noise and RV-derived orbital parameters, the orbital inclination and true mass of these companions.

In Sect. 2, we define the sample of companions and host stars selected from this study. In Sect. 3, we explore the Gaia archive for the selected systems and reduce the sample of companions to those with exploitable Gaia DR1 data and astrometric excess noise. We summarize the GASTON method in Sect. 4. The GASTON results are presented in Sect. 5. These results are then discussed in Sect. 6. We conclude in Sect. 7.

\section{Initial exoplanet candidates selection}

In order to measure the inclination and true mass of orbiting exoplanet candidates, complete information on orbital parameters are required. We thus first need to select a database in which the largest number of published exoplanets fulfil several criteria as follows:

1. A measurement for period $P$, eccentricity $e, \mathrm{RV}$ semiamplitude $K$ and star mass $M_{\star}$ must exist;

2. $K, P$ and $M_{\star}$ should be $>0$;

3. If $e>0.1$, a measurement for $T_{\mathrm{p}}$ and $\omega$ the time of periastron passage and the angle of periastron, respectively, must exist. If $e<0.1$, the orbit is about circular, the phase is not taken into account in the GASTON method and thus $T_{\mathrm{p}}$ and $\omega$ are spurious parameters;

4. A given value for $m \sin i$ (otherwise calculated from other orbital parameters);

5. The date of the last update of the database.

We compared the three main exoplanets databases available online, applying these above criteria: the www.exoplanet.eu (Schneider et al. 2011), www . exoplanets . org (Han et al. 2014), and NASA exoplanet archive. A complete review on the current state of online catalogs has been achieved in Christiansen (2018). The result of this comparison is shown in Table 1.

Although this database has not been updated since June 2018, www.exoplanets.org is the most complete available with respect to planetary, stellar, and orbital parameters, having a complete set of orbital data for 911 companions. For comparison, in the NASA exoplanet archive (NEA), there are only 580 exoplanets for which a complete set of parameters is given. In the Exoplanet. eu database, a reference in terms of up-to-date data (4302 against 4197 in the NEA on 12 August 2020) suffers from inhomogeneities in the reported data; for example, some masses are expressed in Earth mass while most are given in Jupiter mass or the RV semi-amplitudes are only sparsely reported. We found that it is best to rely on the www.exoplanets.org database, which is the most homogeneous even though it only comprises 3262 objects. This database constitutes a robust, yet not too old, reference sample of objects that will remain unchanged in the future since updates have ceased.

In this database, applying the above criteria, the sample of companions reduces down to 924 companions. A measurement of $m \sin i$ is provided with uncertainties for 911 of these companions, following Wright et al. (2011). There thus remains 13 
Table 2. Selected exoplanet companions (see Sect. 2) and orbital parameters from exoplanets . org.

\begin{tabular}{|c|c|c|c|c|c|c|c|c|c|c|}
\hline Companion & $\begin{array}{c}m \sin i \\
\left(M_{\mathrm{J}}\right)\end{array}$ & $\begin{array}{c}P \\
\text { (days) }\end{array}$ & $e$ & $\begin{array}{l}\omega \\
\left(^{\circ}\right)\end{array}$ & $\begin{array}{c}T_{\mathrm{p}} \\
(\mathrm{JD})\end{array}$ & $\begin{array}{c}K \\
\left(\mathrm{~m} \mathrm{~s}^{-1}\right)\end{array}$ & $\begin{array}{c}M_{\star} \\
\left(M_{\odot}\right)\end{array}$ & $\begin{array}{c}\text { Drift flag } \\
(0 / 1)\end{array}$ & $\begin{array}{c}\mathrm{RV}(\mathrm{O}-\mathrm{C}) \\
\left(\mathrm{m} \mathrm{s}^{-1}\right)\end{array}$ & $\begin{array}{c}\text { Transit flag } \\
(0 / 1)\end{array}$ \\
\hline 11 Com b & $16.1284 \pm 1.53491$ & $326.03 \pm 0.32$ & $0.231 \pm 0.005$ & $94.8 \pm 1.5$ & $2452899.6 \pm 1.6$ & $302.8 \pm 2.6$ & $2.04 \pm 0.29$ & 0 & 25.5 & 0 \\
\hline $11 \mathrm{UMi} b$ & $11.0873 \pm 1.10896$ & $516.22 \pm 3.25$ & $0.08 \pm 0.03$ & $117.63 \pm 21.06$ & $2452861.04 \pm 2.06$ & $189.7 \pm 7.15$ & $1.8 \pm 0.25$ & 0 & 28 & 0 \\
\hline 14 And b & $4.68383 \pm 0.22621$ & $185.84 \pm 0.23$ & 0 & 0 & $2452861.4 \pm 1.5$ & $100 \pm 1.3$ & $2.15 \pm 0.15$ & 0 & 20.3 & 0 \\
\hline 14 Her b & $5.21486 \pm 0.298409$ & $1773.4 \pm 2.5$ & $0.369 \pm 0.005$ & $22.6 \pm 0.9$ & $2451372.7 \pm 3.6$ & $90 \pm 0.5$ & $1.066 \pm 0.091$ & 0 & 5.6 & 0 \\
\hline 16 Cyg B b & $1.63997 \pm 0.0833196$ & $798.5 \pm 1$ & $0.681 \pm 0.017$ & $85.8 \pm 2$ & $2446549.1 \pm 7.4$ & $50.5 \pm 1.6$ & $0.956 \pm 0.0255$ & 0 & 7.3 & 0 \\
\hline 18 Del b & $10.298 \pm 0.36138$ & $993.3 \pm 3.2$ & $0.08 \pm 0.01$ & $166.1 \pm 6.5$ & $2451672 \pm 18$ & $119.4 \pm 1.3$ & $2.33 \pm 0.05$ & 0 & 15.5 & 0 \\
\hline 24 Boo b & $0.912932 \pm 0.110141$ & $30.3506 \pm 0.00775$ & $0.042 \pm 0.0385$ & $210 \pm 115$ & $2450008.6 \pm 9$ & $59.9 \pm 3.25$ & $0.99 \pm 0.16$ & 0 & 0.02651 & 0 \\
\hline $24 \mathrm{Sex} b$ & $1.83564 \pm 0.108126$ & $455.2 \pm 3.2$ & $0.184 \pm 0.029$ & $227 \pm 20$ & $2454758 \pm 30$ & $33.2 \pm 1.6$ & $1.81 \pm 0.08$ & 0 & 4.8 & 0 \\
\hline 24 Sex c & $1.51716 \pm 0.200171$ & $910 \pm 21$ & $0.412 \pm 0.064$ & $352 \pm 9$ & $2454941 \pm 30$ & $23.5 \pm 2.9$ & $1.81 \pm 0.08$ & 0 & 6.8 & 0 \\
\hline$\ldots$ & $\ldots$ & $\ldots$ & $\ldots$ & $\ldots$ & $\ldots$ & $\ldots$ & $\ldots$ & $\ldots$ & $\ldots$ & $\ldots$ \\
\hline
\end{tabular}

Notes. Only the 9 first companions of the sample are shown. The whole sample of 912 companions is available at the CDS. The reference papers of orbital and stellar parameters given in this table are cited in the exoplanets.org database.

objects for which the $m \sin i$ was not provided. Those planets are all transiting, but for 12 of these objects no RV signal is detected (Marcy et al. 2014) and $K$ is only a Markov chain Monte Carlo (MCMC) estimation with large error bars. We exclude those 12 objects from our analysis. The remaining planet with no $m \sin i$ given in the database is Kepler-76 b. However, a solid RV-variation detection was reported in Faigler et al. (2013), leading to an $m \sin i$ of $2 \pm 0.3 M_{\mathrm{J}}$. We thus keep Kepler-76 b in our list of targets and insert its $m \sin i$ measurement.

The selected sample also includes 358 exoplanets detected with transit photometry and Doppler velocimetry. These companions with known inclination of their orbit - edge-on in virtually all cases - are useful to assess the quality of the inclinations obtained independently with GASTON. The full list of 912 selected companions orbiting 782 host stars are shown in Table 2.

\section{Gaia inputs}

\subsection{Gaia DR1 data for the target list}

The GASTON algorithm determines the inclination of RV companion orbits using the Gaia DR1 astrometric excess noise (Gaia Collaboration 2016; Lindegren et al. 2016). The most recent Gaia DR2 release cannot be used similarly because it is based on a different definition of the astrometric excess noise and moreover cursed by the so-called DOF bug, directly affecting the measurement of residual scatter (Lindegren et al. 2018). For that reason, from Kiefer et al. (2019) it was decided to rely the GASTON analysis, even though this analysis uses preliminary Gaia DR1 data.

The list of host stars constituted in Sect. 2 is uploaded in the Gaia archive of the DR1 to retrieve astrometric data around each star, with a search radius of 5". Among the 782 host stars of our initial sample defined in the previous section, we found 679 entries in the DR 1 catalog. Most stars are reported singles, but among the 679 DR1 sources, 44 (with 50 reported exoplanets) have a close background star, a visual companion, or a duplicated (but non-identified) source, with a separation to the main source smaller than 5".

In particular, seven stars (with 12 reported exoplanets) have a "visual companion" with a different ID, at less than 5" distance but with an equal magnitude \pm 0.01 . This is strongly suspicious and must be due to duplication in the catalog. Duplication is only reported in the Gaia DR1 database for one of those sources, YZ Cet. We consider it safer to exclude these seven sources from our analysis. However, in general we want to keep those that are denoted as duplicates. Duplication separates the dataset of a single source into two different IDs. In the worst case scenario, duplication lead to ignore outlying measurements and thus to underestimate the astrometric scatter. This can only be problematic if GASTON leads us to characterize a mass in the regime of planets, since underestimating the astrometric excess noise implies underestimating the mass. More generally, duplication is not an issue because GASTON characterizes masses in the regime of BD or stars, allowing us to exclude a planetary nature.

Finally, we identified three supplementary problematic hosts that have a magnitude difference with commonly adopted values (as in, for example, SIMBAD) larger than 3. These are Proxima Cen, HD 142 and HD 28254 (see, e.g., Lindegren et al. 2016 for Proxima Cen). We also excluded these from our studied sample. We also note the presence of 11 sources with a null parallax, which are also taken off the sample.

The Gaia DR1 sources are divided into two different datasets: the primary and the secondary (Lindegren et al. 2016). The primary dataset contains two million of the targets also observed with Tycho/HIPPARCOS, for which there is a robust measurement of parallax and proper motion out of a 24-yr baseline astrometry. This is also sometimes referred to as the joint Tycho-Gaia Astrometric Solution (TGAS) dataset. The secondary dataset contains 1.141 billion sources that do not have a supplementary constraint on position from Tycho/HIPPARCOS, some of which are also newly discovered objects. In the secondary dataset, the proper motion and parallax are fitted to the Gaia data, leaving from a prior based on magnitude (Michalik et al. 2015b), but they are discarded in the DR1. In Lindegren et al. (2016), it is reported that the astrometric residuals scatter is generally larger in the secondary dataset that in the primary dataset (see also Sect. 3.3). We thus separate those secondary dataset objects from those in the primary dataset in the rest of the study and treat the secondary dataset objects specifically.

In total, we constituted a sample of 755 exoplanets with both RV and Gaia DR1 data, orbiting 658 stars of which Table 3 gives the full list. Among those, 508 exoplanets orbit 436 stars in the primary dataset, for 247 exoplanets around 222 stars in the secondary dataset. We list among all DR1 parameters the $G$-band magnitude, the parallax, belonging to primary or secondary dataset, source duplication (see, e.g., 
A\&A 645, A7 (2021)

Table 3. Selected stellar hosts from the initial sample and selected from the Gaia DR1 archive (see Sect. 3.1).

\begin{tabular}{|c|c|c|c|c|c|c|c|c|c|c|c|c|c|c|}
\hline \multirow[t]{2}{*}{ Source } & \multicolumn{5}{|c|}{ SIMBAD } & \multicolumn{8}{|c|}{ Gaia DR1 } & \multirow{2}{*}{$\begin{array}{c}\text { Gaia DR2 } \\
b-r^{(i)}\end{array}$} \\
\hline & RA & Dec & $V$ & $B-V$ & Sp type & $\overline{\mathrm{G}^{(a)}}$ & $\begin{array}{c}\pi^{(b)} \\
(\mathrm{mas})\end{array}$ & Dataset $^{(c)}$ & Duplicate $^{(d)}$ & $N_{\mathrm{FoV}}{ }^{(e)}$ & $N_{\text {tot }}(f)$ & $\begin{array}{c}\varepsilon_{\mathrm{DR} 1}{ }^{(g)} \\
(\mathrm{mas})\end{array}$ & $\overline{D_{\varepsilon}{ }^{(h)}}$ & \\
\hline $11 \mathrm{UMi}$ & $15: 17: 05.8915$ & $+71: 49: 26.0375$ & 5.02 & 6.38 & K4III & 4.7 & $7.47 \pm 0.66$ & 1 & False & 15 & 76 & 2.4 & 11220 & 1.51 \\
\hline 14 And & $23: 31: 17.4127$ & $+39: 14: 10.3105$ & & 6.24 & G8III & 5.0 & 13.23 & 2 & False & 6 & 49 & 5.6 & 14395 & 1.17 \\
\hline $14 \mathrm{Her}$ & $16: 10: 24.3153$ & $+43: 49: 03.4987$ & & 7.57 & KOV & 6.3 & $55.93 \pm 0.24$ & 1 & False & 18 & 107 & 0.62 & 258 & 1.00 \\
\hline 16 Cyg B & $19: 41: 51.9732$ & $+50: 31: 03.0861$ & 6.20 & 6.86 & G3V & 6.0 & $47.12 \pm 0.23$ & 1 & False & 15 & 80 & 0.40 & 173 & 0.83 \\
\hline 18 Del & $20: 58: 25.9337$ & $+10: 50: 21.4261$ & 5.51 & 6.43 & G6III & 5.3 & 13.09 & 2 & False & 7 & 50 & 3.0 & 10385 & 1.08 \\
\hline 24 Boo & $14: 28: 37.8131$ & $+49: 50: 41.4611$ & & 6.44 & G4III-IVFe-1 & 5.3 & $10.23 \pm 0.56$ & 1 & False & 31 & 195 & 2.6 & 5721 & 1.07 \\
\hline $24 \mathrm{Sex}$ & $10: 23: 28.3694$ & $-00: 54: 08.0772$ & 6.44 & 7.40 & KOIV & 6.1 & 13.85 & 2 & False & 6 & 44 & 0.68 & 175 & 1.10 \\
\hline 30 Ari B & $02: 36: 57.7449$ & $+24: 38: 53.0027$ & 7.09 & 7.59 & F6V & 6.9 & $21.42 \pm 0.60$ & 1 & False & 10 & 71 & 1.8 & 428 & 0.68 \\
\hline $7 \mathrm{CMa}$ & $06: 36: 41.0376$ & $-19: 15: 21.1659$ & 3.91 & 5.01 & K1.5III-IVFe1 & 4.0 & 50.63 & 2 & False & 37 & 272 & 6.0 & 164838 & 1.24 \\
\hline $70 \mathrm{Vir}$ & $13: 28: 25.8082$ & $+13: 46: 43.6430$ & 4.97 & 5.68 & G4Va & 4.9 & $54.70 \pm 0.88$ & 1 & False & 29 & 213 & 3.2 & 11554 & 0.90 \\
\hline$\ldots$ & $\ldots$ & $\ldots$ & $\ldots$ & $\ldots$ & $\ldots$ & $\ldots$ & $\ldots$ & $\ldots$ & $\ldots$ & $\ldots$ & $\ldots$ & $\ldots$ & $\ldots$ & $\ldots$ \\
\hline
\end{tabular}

Notes. Only the 10 first sources of the sample are shown. The whole list of 658 stars hosting 755 exoplanet candidates is available at the CDS. ${ }^{(a)}$ The Gaia recorded flux magnitude in the $G$ band. ${ }^{(b)}$ The parallax. For the sources from the secondary dataset, the values are given without error bars since they are missing from the DR1. These values are taken from the DR2. For those, we assume $10 \%$ error bars in the rest of the study. ${ }^{(c)}$ DR1 primary (1) or secondary (2) dataset. ${ }^{(d)}$ Duplicate source (true) or not (false), as explained in Lindegren et al. (2016). ${ }^{\left({ }^{(e)}\right.}$ Number of field-of-view transits of the sources (matched_observations in the DR1 database). ${ }^{(f)}$ Total number of astrometric AL observations reported (astrometric_n_good_obs_al in the DR1 database). ${ }^{\left({ }^{g}\right)}$ Astrometric excess noise in mas. ${ }^{\left({ }^{h}\right)}$ Significance of $\varepsilon_{\text {DR } 1}$. Any $D_{\varepsilon}>2$ leads to a significant astrometric excess noise with $p$-value $=e^{-D_{\varepsilon} / 2}$ as explained in Lindegren et al. (2012). ${ }^{(i)} G_{\mathrm{b}}-G_{\mathrm{r}}$ color index as presented in Lindegren et al. (2018).

Lindegren et al. 2016), number of field-of-view transits $N_{\text {FoV }}$ (matched_observations in the DR1 catalog), total number of recorded along-scan angle (AL) measurements $N_{\text {tot }}$, astrometric excess noise $\varepsilon_{\mathrm{DR} 1}$, and its significance parameter $D_{\varepsilon}$ (Lindegren et al. 2012).

\subsection{Magnitude, color, and parallax correlations with astrometric excess noise}

The astrometric excess noise is the main measured quantity that is used in this study to derive a constraint on the RV companion masses listed in Table 2. The fundamental hypothesis assumed in GASTON relates the astrometric excess noise to astrometric orbital motion. It is thus crucial to identify possible systematic correlations of this quantity with respect to other intrinsic data such as magnitude, color, or DR1 dataset, which would reveal instrumental or modeling effects.

As can be seen in Fig. 1, stars brighter than magnitude 6.4 show a significant drift of increasing excess noise with decreasing magnitude. This is a sign of instrumental systematics, such as point spread function, jitter, and charge-coupled device (CCD) sensibility, that are recognized to occur in Gaia data (Lindegren et al. 2018). With $G$-mag $<6.4$, the astrometric excess noise are all larger than 0.4 mas, with a median value about 0.7 mas. In the rest of the paper, we thus exclude any source with a $G$-mag $<6.4$, reducing the sample to 614 sources.

Moreover, the astrometric excess noise also shows a correlation with color indices, that is, the B-V as found in Simbad for 498 sources with $G$-mag $>6.4$, and the Gaia DR2 $b-r$ available in the DR2 database for all the 614 sources with $G$-mag $>6.4$, as plotted in Figs. 2 and 3. A moving median filter of the data shows a correlation of $\varepsilon_{\mathrm{DR} 1}$ with $\mathrm{B}-\mathrm{V}$ beyond 1 , and with DR2 $b-r$ beyond 1.4. The $B-V$ index is not available for all the 614 sources, we thus prefer to use the DR2 $b-r$ index as a limiting parameter. As for the magnitude, the astrometric excess noise is larger than 0.4 mas whatever $b-r$ larger than 1.8. A correlation of the AL angle residuals with $V-I$ color was already reported

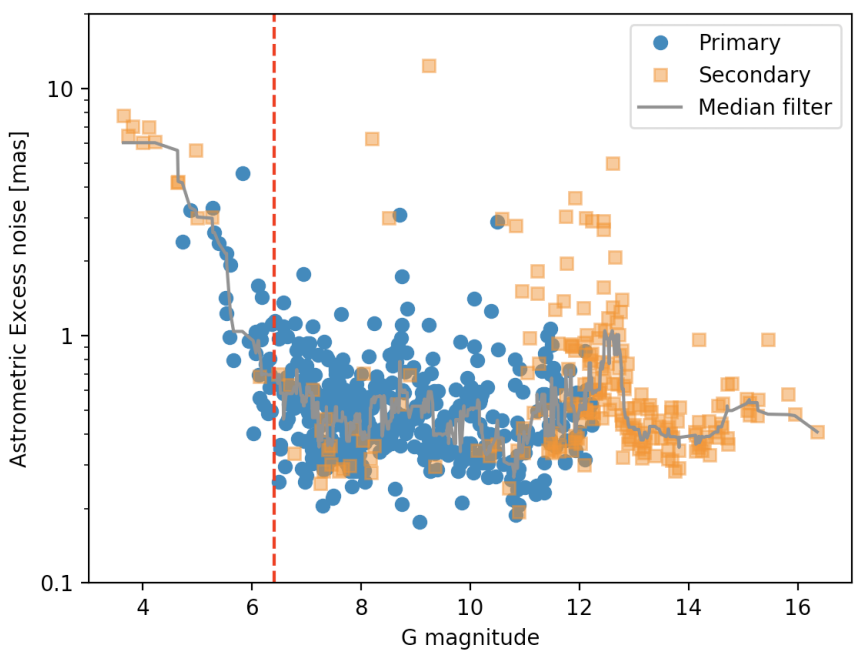

Fig. 1. Comparing astrometric excess noise to $G$ magnitude for the 658 stars of Table 3 . The red dashed line shows the $G=6.4$ limit discussed in the text. We separate targets from the primary dataset (blue) and from the secondary dataset (orange). The gray line indicates a moving median filter of the data.

in Appendix D.2 of Lindegren et al. (2016). These two correlations likely have a common optical origin due to the chromaticity of the star centroid location on the CCD. In the rest of the paper, we thus also exclude any source with a $b-r>1.8$, reducing the sample to 580 sources.

Figure 4 shows that the parallax and magnitude are correlated in both primary and secondary datasets, which is not surprising because we expect distant sources to be on average less luminous than sources close by. Sources of the secondary dataset are located much farther away from the Sun than sources of the primary dataset, which is expected from the absence of Tycho data for the secondary dataset. The astrometric excess noise is not correlated with parallax, but we observe astrometric excess noise 
F. Kiefer et al.: Determining the true mass of radial-velocity exoplanets with Gaia
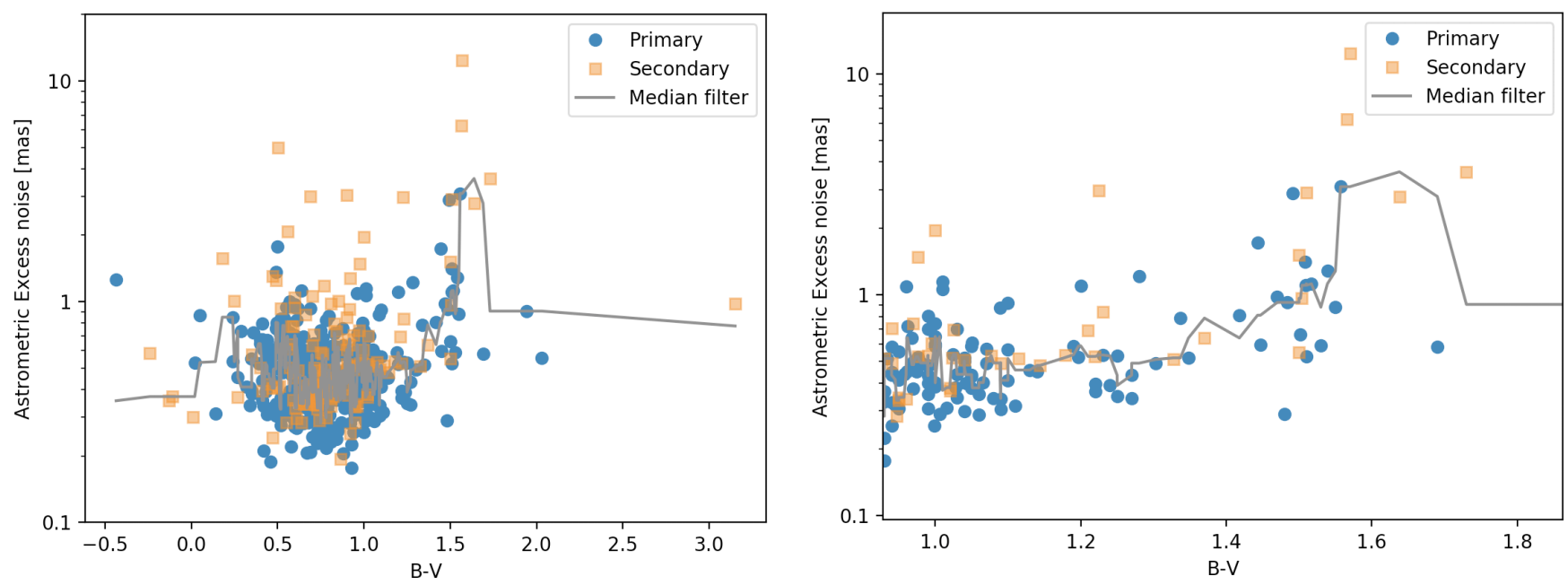

Fig. 2. Astrometric excess noise with respect to $B-V$ for 498 sources with $B-V$ measurements and $G>6.4$. The right panel represents a magnification of the region with positive correlation beyond $B-V=1$. Symbols and color are similar to Fig. 1.
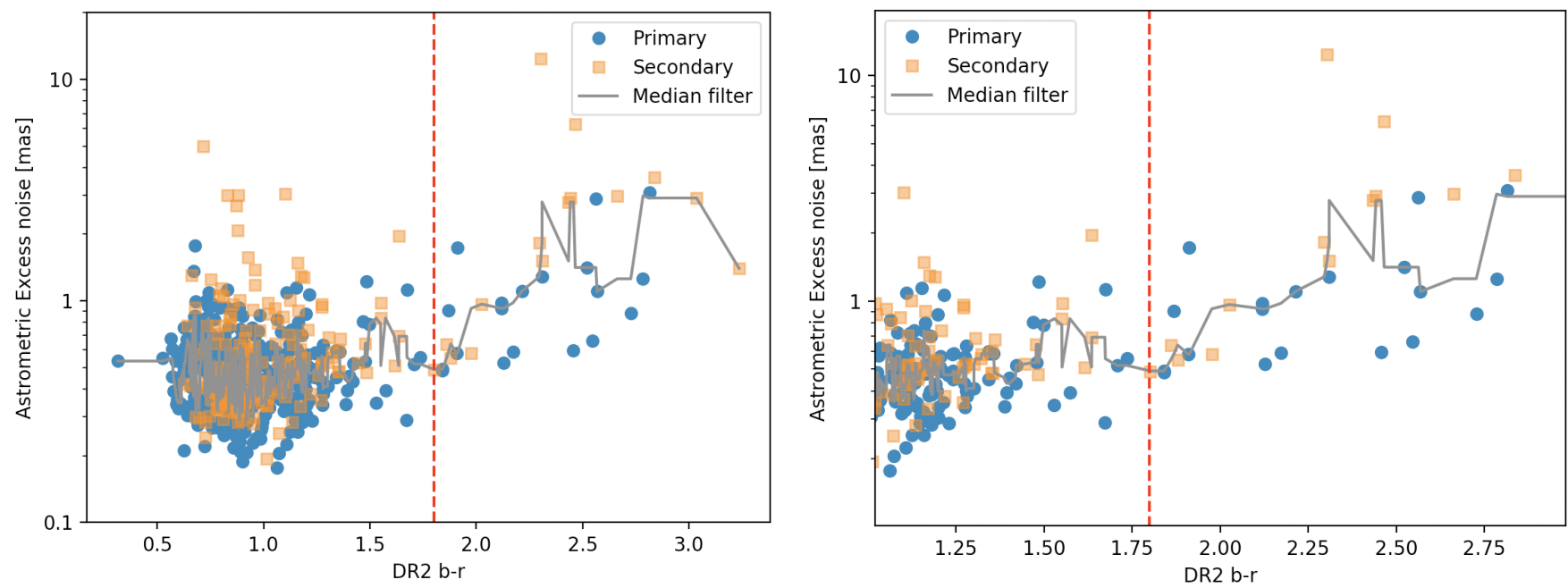

Fig. 3. Astrometric excess noise with respect to $b-r$ color index from Gaia DR2 for the 614 sources with $G>6.4$. The right panel represents a magnification of the region with a positive correlation around the limiting color $b-r=1.8$ (red dashed line). Symbols and color are similar to Fig. 1.
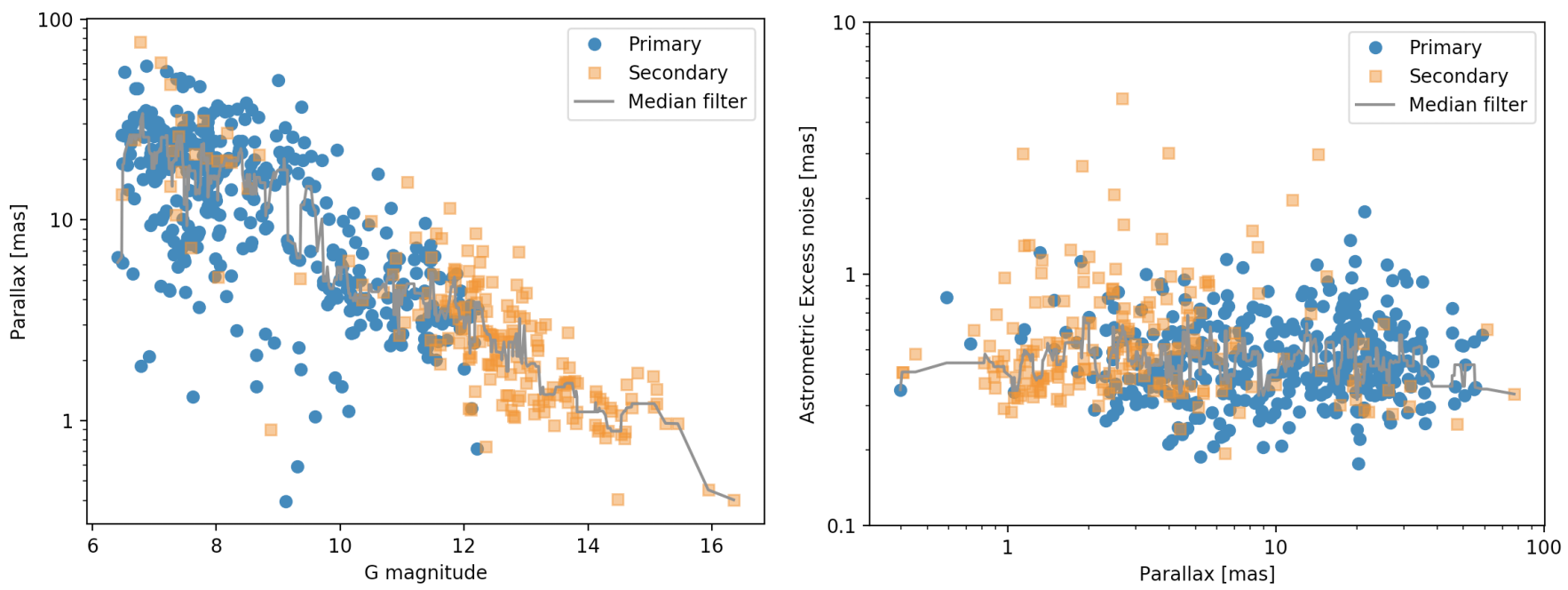

Fig. 4. $G$-magnitude with respect to parallax (left panel) and parallax with respect to astrometric excess noise (right panel) for the 580 sources with $G>6.4$ and $b-r<1.8$. Symbols and color are similar to Fig. 1 . 
Table 4. Distribution of astrometric excess noise with respect to primary, secondary, and HIPPARCOS datasets in the Gaia DR1 database, and varying selection criteria as explained in the text.

\begin{tabular}{|c|c|c|c|c|c|c|}
\hline \multirow[t]{2}{*}{ Reference of data } & \multirow[t]{2}{*}{ DR1 dataset } & \multirow[t]{2}{*}{ Subset } & \multirow[t]{2}{*}{$N_{\text {star }}$} & \multicolumn{3}{|c|}{ Astrometric excess noise } \\
\hline & & & & $\begin{array}{l}\text { 10th-percentile } \\
\text { (mas) }\end{array}$ & $\begin{array}{l}\text { Median } \\
\text { (mas) }\end{array}$ & $\begin{array}{c}\text { 90th-percentile } \\
\text { (mas) }\end{array}$ \\
\hline \multirow{3}{*}{$\begin{array}{l}\text { Lindegren et al. (2016) } \\
\text { (1 } 142719769 \text { stars) }\end{array}$} & Primary & & 2057050 & 0.299 & 0.478 & 0.855 \\
\hline & Secondary & & 1140662719 & 0.000 & 0.594 & 2.375 \\
\hline & HIPPARCOS & & 93635 & 0.347 & 0.572 & 1.185 \\
\hline \multirow{12}{*}{$\begin{array}{l}\text { This paper sample (Table } 3 \text { ) } \\
\text { (524 stars) }\end{array}$} & Primary & & 385 & 0.291 & 0.451 & 0.751 \\
\hline & & All transiting planets & 133 & 0.271 & 0.399 & 0.704 \\
\hline & & $>1$ non-transiting planet & 252 & 0.304 & 0.466 & 0.786 \\
\hline & & With RV drift & 46 & 0.296 & 0.431 & 0.633 \\
\hline & & No RV drift & 339 & 0.291 & 0.453 & 0.761 \\
\hline & Secondary & & 139 & 0.316 & 0.423 & 0.776 \\
\hline & & All transiting planets & 113 & 0.336 & 0.438 & 0.791 \\
\hline & & $>1$ non-transiting planet & 26 & 0.283 & 0.360 & 0.701 \\
\hline & & With RV drift & 7 & 0.297 & 0.334 & 0.511 \\
\hline & & No RV drift & 132 & 0.318 & 0.425 & 0.794 \\
\hline & HIPPARCOS & & 246 & 0.307 & 0.466 & 0.784 \\
\hline & & Including $G<6.4 \& b-r>1.8$ & 297 & 0.324 & 0.513 & 1.048 \\
\hline
\end{tabular}

measurements on the same order, and even larger, than the parallax for sources of the secondary dataset. This strongly suggests issues with parallax and proper motion modeling, reminding us that those parameters are poorly fitted from rough priors in the secondary dataset. We thus discard from the rest of the study secondary sources for which $\log \pi \sim \log \varepsilon_{\mathrm{DR} 1} \pm 0.5$. We think it is wiser to postpone their thorough analysis to the future Gaia DR3 release. Moreover, the largest $\varepsilon_{\mathrm{DR} 1}$ in the secondary dataset are generally obtained for small parallax ( $<10$ mas). This behavior is different from what is observed in the primary dataset for which the astrometric excess noise is not correlated with parallax.

The final sample contains 597 planet candidates. They orbit around 524 host stars with $G>6.4, b-r<1.8$. Sources in the secondary dataset also fulfil $\log \pi-\log \varepsilon_{\mathrm{DR} 1}>0.5$.

\subsection{Distribution of astrometric excess noise}

To get a sense of how $\varepsilon_{\mathrm{DR} 1}$ is a relevant quantity to characterize a binary or planetary system, it is crucial to understand how the astrometric excess noise generally varies with respect to the known or unknown inclination of the gravitational systems observed, transiting or not; with respect to the presence of a long-period outer companion in the system; with respect to the presence of RV drift; and with respect to the quality of the observations of these systems with Gaia, whether from primary or secondary dataset. We perform an analysis of the distribution of astrometric excess noise of our selected sample of companions and sources as defined in Sect. 3.2, with respect to following subsets selection criteria:

- Dataset (primary/secondary);

- All planets around the host star are transiting;

- At least one planet is not transiting;

- Detection or hint of an RV drift;

- No hint of an RV drift.

In principle, with orbital inclination fixed to $\sim 90^{\circ}$, the semimajor axis of transiting planets host stars should not reach more than a few $\mu$ as, and remain undetectable in the DR1 astrometric excess noise. The astrometric scatter is dominated by the instrumental and measurement noises on the order of $\sim 0.6$ mas (Lindegren et al. 2016). The distribution of $\varepsilon_{\mathrm{DR} 1}$ for transiting planet hosts should be close to the distribution of astrometric scatter due to pure instrumental and measurement noises. On the other hand, systems with non-transiting planets allow inclinations down to $0^{\circ}$, and host-star semimajor axes beyond a few 0.1 mas. We expect their astrometric excess noise to be generally larger than for systems with transiting-only planets. Finally, the detection of a drift in the RV suggests the presence of a hidden outer companion in the system. The astrometric excess noise might be systematically larger for those systems, implying that the astrometric signal is not only due to the companion with a well-defined orbit. This is however certainly not a rule, as shown in the case of HD 114762, for instance (Kiefer 2019), for which the astrometric excess noise is dominated by the effect of the short period companion HD $114762 \mathrm{Ab}$.

In Table 4, we present the 10th, 50th, and 90th percentiles of the astrometric excess noise distribution according to the different subsamples defined above. We confront these to the Lindegren et al. (2016) percentiles derived for the whole primary, secondary, and HIPPARCOS DR1 datasets (Tables 1 and 2 in Lindegren et al. 2016) based on more than 1 billion sources observed with Gaia. In the first panel of Fig. 5, we compare the distributions of astrometric excess noise for the sources from the primary and secondary datasets with transiting-only planets; in the second panel, we compare sources from the primary dataset with transiting-only planets to those with at least one non-transiting planet.

The median and 90th percentile values of the $\varepsilon_{\mathrm{DR} 1}$ distribution for all subsets in the primary dataset are generally compatible with the Lindegren et al. (2016) values. Although the Lindegren et al. (2016) study shows that the HIPPARCOS subset is associated with larger excess noise, Table 4 shows that excluding $G$-mag $>6.4$ and $b-r>1.8$ objects as proposed in Sect. 3.1 leads to decreasing the extent of the astrometric excess 

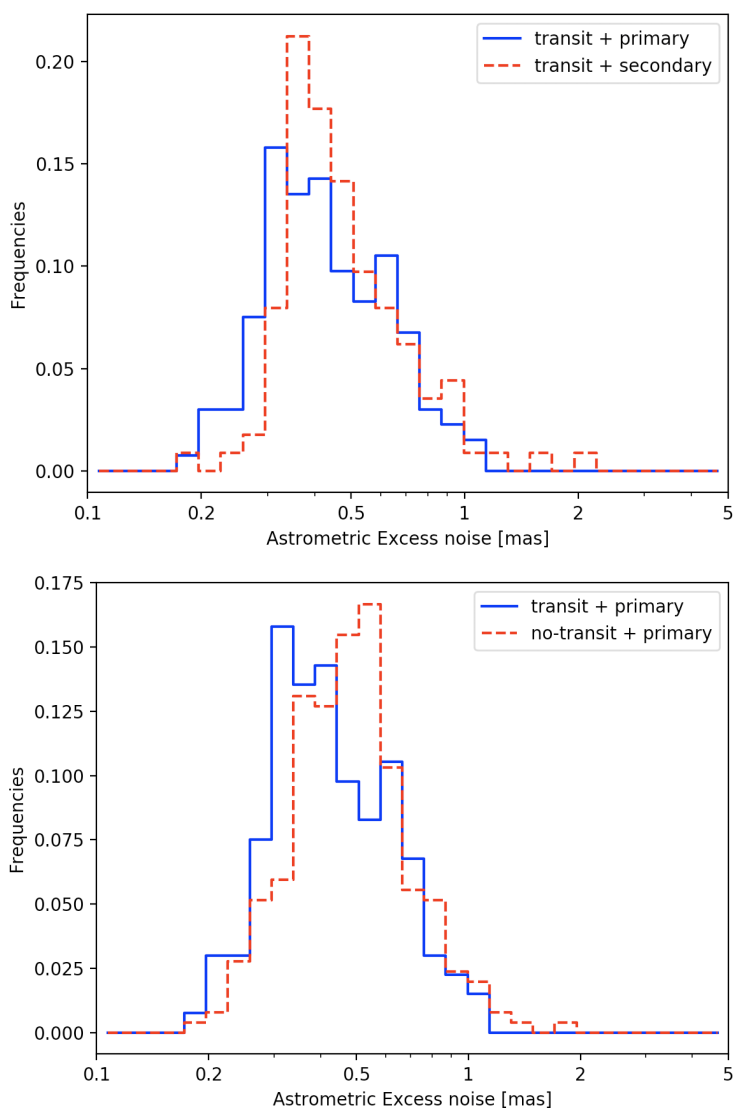

Fig. 5. Top panel: astrometric excess noise distribution for sources with only transiting planets, comparing the primary and secondary datasets. Bottom panel: comparing "only transiting" and "non-transiting" subsets within the primary dataset.

noise distribution with values agreeing with the primary dataset. The HIPPARCOS subset excluding bright and late-type sources is thus likely not different from the full primary dataset.

We observe a clear distinction in the distributions of $\varepsilon_{\mathrm{DR} 1}$ between the primary and secondary datasets, with significantly higher astrometric excess noise in the secondary dataset. This could be explained well by the absence, for the secondary dataset, of the Tycho/HIPPARCOS supplementary positions $24 \mathrm{yr}$ ago that allows the derivation of robust proper motion and parallax for the sources in the primary dataset. The derivation of proper motion and parallax from Gaia data only with Galactic priors based on magnitude (Michalik et al. 2015b; Lindegren et al. 2016) certainly leads to larger scatter in the residuals of the five-parameter solution.

For transiting sources of the primary dataset, the 90th percentile of the astrometric excess noise distribution is 0.70 mas. This is compatible with, and even lower than, Lindegren et al. (2016) values of the global DR1 solution. For this subset, the 95th percentile is 0.81 mas, still lower than the 90th percentile of Lindegren et al. (2016). These generally small astrometric excess noises of the sources with transiting planets are compatible with statistical noise and the non-detection by Gaia of any orbital motion of a star orbited by a planet at short separation $(<0.1 \mathrm{au}$ or $P<50$ days) and with an edge-on inclination of its orbit.

The systems in the primary dataset with a non-transiting planet have the highest median among all other subsets (0.47 mas) and the highest 90th percentile (0.78 mas). More importantly, the astrometric excess noise of sources with nontransiting companions is significantly larger than for sources with transiting-only planets. This can also be seen in the bottom panel of Fig. 5 with a net shift between the two $\varepsilon_{\mathrm{DR} 1}$ distributions. This confirms that $\varepsilon_{\mathrm{DR} 1}$ contains a non-negligible fraction of astrometric motion for systems with a companion whose orbital inclination is not known.

The $\varepsilon_{\mathrm{DR} 1}$ in the secondary dataset generally reaches larger values than in the primary dataset, with a 90th percentile for the subset of systems with transiting-only planets $\sim 0.8$ mas. This was expected by the less accurate fit of the proper motion and parallax in the secondary dataset compared to the primary. However, this is also much smaller than the 2.3 mas 90th percentile derived for the whole secondary dataset in Lindegren et al. (2016). Therefore, once cleaned from problematic systems, in particular those with $\log \pi / \varepsilon_{\mathrm{DR} 1}>0.5$ (Sect. 3.2), the astrometric excess noise of remaining objects in the secondary dataset seems robust, with parallax and proper motion most likely well determined (although not published in the DR1).

Interestingly, we find no correlation of the astrometric excess noise distribution with the presence of any drift in the RV data, and even smaller values than in the other subsets. This could be a consequence of the smaller number of sources in this category, which if following an inclination probability density function $(\mathrm{PDF}) \sim \sin I_{\mathrm{c}}$ would preferentially have inclinations close to $90^{\circ}$, and thus smaller astometric motion. It also suggests that the presence of an outer companion does not have a strong effect on the astrometric excess noise compared to the enhanced astrometric motion owing to the small inclination of a non-transiting companion.

\subsection{Testing the noise model}

From the 133 and 113 stars with transiting-only planets from the primary and secondary datasets, respectively, we can test the model of noise used in the simulations of GASTON. In previous studies (Kiefer et al. 2019; Kiefer 2019), we chose to use values based on published estimations of the measurement uncertainty and typical external noise (including modeling noise and instrument jitter) of $\sigma_{\mathrm{AL}}=0.4$ mas (Michalik et al. 2015a) and $\sigma_{\text {syst }}=0.5$ mas, respectively (Lindegren et al. 2016). As we showed in the preceding section, the sources with transiting companion must be generally more similar to sources with no astrometric motion. Therefore, the astrometric excess noise measured by Gaia for these sources should be close to purely instrumental and photonic stochastic scatter.

The distribution of $\varepsilon_{\mathrm{DR} 1}$ for these 300 sources from primary and secondary datasets is plotted in Fig. 6. It is compared to simulations of astrometric excess noise of sources with no orbital motion in the framework of various noise models. We assumed for each simulation random numbers of FoV transits $\left(N_{\mathrm{FoV}}\right)$ and numbers of measurements per FoV transit $\left(N_{\mathrm{AL}}\right)$ in the same ranges as those of the sample presented here (e.g., $N_{\mathrm{FoV}}=15 \pm 8$ and $N_{\mathrm{AL}}= \pm 2$ with Gaussian distribution), and imposing that $48>N_{\mathrm{FoV}}>5$ and $9>N_{\mathrm{AL}}>2$. We singled out five different noise models of $\left(\sigma_{\text {syst }}, \sigma_{\mathrm{AL}}\right)$, either based on literature values or based on the best fit of a bi-uniform distribution of $\sigma_{\text {syst }}$ with a fixed median to the $\varepsilon_{\mathrm{DR} 1}$ cumulative density function (cdf) as follows:

- The constant model for the primary dataset, as used in Kiefer et al. (2019) and Kiefer (2019): $\sigma_{\mathrm{AL}}=0.4$ mas from Michalik et al. (2015a) and $\sigma_{\text {syst }}=0.5$ mas (based on Lindegren et al. 2016);

- A different constant model for the secondary dataset: $\sigma_{\mathrm{AL}}=$ 0.4 mas as above and $\sigma_{\text {syst }}=0.6$ mas (based on Lindegren et al. 2016); 

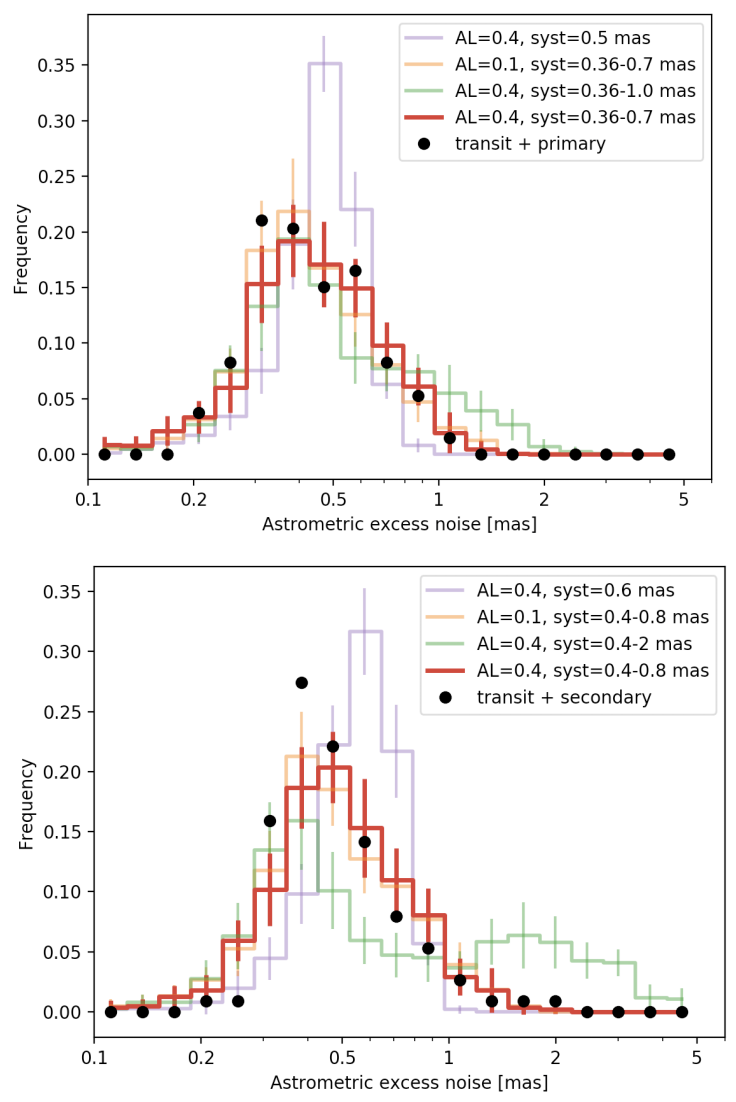

Fig. 6. Comparing measurement and systematically noise models to the distribution of astrometric excess noise measurement for sources with transiting companion in the primary dataset (top panel) and in the secondary dataset (bottom panel). See text for explanations.

- Random $\sigma_{\text {syst }}$ from a distribution with a median at 0.4 mas, uniform from 0.36 to 0.40 mas, and from 0.4 to 0.7 mas for the primary Dataset;

- Random $\sigma_{\text {syst }}$ from a distribution with a median at 0.45 mas, uniform from 0.4 to 0.45 mas, and from 0.45 to 0.8 mas for the secondary Dataset;

- Smaller AL angle measurement uncertainty as suggested from Lindegren et al. (2018): $\sigma_{\mathrm{AL}}=0.1$ mas.

The bi-uniform distributions models were found to lead to the best least-squares fit of the observed $\varepsilon_{\mathrm{DR} 1}$ cdf. All five models are compared to the data in Fig. 6. A model with a wide range of systematic noise better explains the observed distributions for values of astrometric excess noise assumed to be compatible with pure stochastic and systematic noise, for example, below 0.85 mas and 2.3 mas for sources in the primary and secondary datasets, respectively. The constant noise model tends to overestimate the astrometric excess noise, and the simulated distribution decreases too steeply at values closer to 1 mas. The noise $\sigma_{\text {syst }}$ taken from a bi-uniform distribution of values within bounds (e.g., $0.36-0.7$ mas in the primary dataset) explains the full distribution of observed astrometric excess noises better. The distribution is damped beyond about $0.85-1$ mas for the primary dataset and $\sim 1.2-1.4$ mas in the secondary dataset, with less than $1 \%$ of the simulations beyond.

We can exclude that the $\sigma_{\text {syst }}$ is much larger than 0.7 mas in the primary dataset, and 0.8 mas in the secondary dataset, since that would extend the core of the distribution toward larger excess noise, therefore leading to a poorer agreement. Finally, we observe that the AL angle measurement uncertainty $\sigma_{\mathrm{AL}}$ does not have a strong impact on the astrometric excess noise.
We tested two values: a small uncertainty 0.1 mas, as given in Lindegren et al. (2018); and a more conservative value, as assumed by Michalik et al. (2015a) of 0.4 mas, which corresponds well to the typical AL residuals reported in Lindegren et al. (2016) of 0.65 mas $\left(\sqrt{\left(\sigma_{\mathrm{AL}}=0.4\right)^{2}+\left(\sigma_{\text {syst }}=0.5\right)^{2}}=\right.$ 0.64 mas). We thus fix $\sigma_{\mathrm{AL}}$ to 0.4 mas.

In conclusion, the systematic noise is typically about 0.5 mas and 0.6 mas in the primary and secondary dataset, respectively. But it is likely that for an individual observed source, $\sigma_{\text {syst }}$ can be somewhat larger or smaller by a few fraction of mas. We thus adopt a random systematic noise for each simulation in GASTON uniformly distributed on both sides of the median at 0.4 mas down to 0.36 mas and up to 0.7 mas for sources in the primary dataset, and about the median at 0.45 mas down to 0.4 mas and up to 0.8 mas for sources in the secondary dataset.

\subsection{Detection threshold and source selection}

The GASTON tool cannot be used to characterize the mass of as much as 597 planet candidates. This would require several weeks of calculation, while many of these candidates cannot be truly characterized, because the astrometric excess noise is compatible with an edge-on inclination. We thus need to define a robust threshold above which $\varepsilon_{\mathrm{DR} 1}$ can be considered significantly non-stochastic - and thus astrophysical - and below which this threshold could be explained by pure stochastic noise.

Given that close to $40-50 \%$ of the sources in the Milky Way are part of binary systems (Duquennoy \& Mayor 1991; Raghavan et al. 2010), a consequent fraction of Gaia sources - possibly more than $10 \%$ - could show a detectable astrometric motion. We thus consider using the 90th percentiles of Lindegren et al. (2016) based on a sample of more than 1 billion stars, as a detection threshold, above which a significant fraction of $\varepsilon_{\mathrm{DR} 1}$ could be imputed to astrometric motion. This was assumed in previous work (Kiefer 2019), but the astrometric excess noise distribution of the present sample might differ from the sample upon which these percentiles were calculated.

In Sects. 3.3 and 3.4, we showed that the 90th percentile of $0.85 \mathrm{mas}$ in the primary sample as derived in Lindegren et al. (2016) is robust, but the 2.3 mas threshold for secondary dataset sources is excessive and could be more reasonably lowered to $\sim 1.2$ mas. This overestimation of the 90th percentile in Lindegren et al. (2016) must be due to the inclusion of small magnitude, large $b-r$ color sources and badly modeled parallax, which we did clean out from our sample. We thus use the detections thresholds $\epsilon_{\text {thresh,prim }}=0.85$ mas and $\epsilon_{\text {thresh,sec }}=1.2$ mas, above which the astrometric excess noise would be mainly due to supplementary astrometric motion. This reduces our sample to 28 sources (29 planet candidates) with an astrometric detection by Gaia in the DR1. These are the best candidates for orbit inclination and true mass measurement of the companion. They constitute the "detection sample".

We counted 312 non-transiting planet candidates (around 254 sources) for which the inclination is not constrained from photometry and for which the astrometric motion of their host star leads to an astrometric excess noise smaller than the threshold. Even though it is compatible with pure noise, the astrometric excess noise allows us to constrain the true astrometric extent of the orbit of the star. This leads us to derive a minimum inclination and a maximum true mass of the exoplanet candidates beyond which they are not compatible with a non-detection. In this sample, we exclude the so-called duplicate sources in the Gaia DR1 because, for such target, possibly large sets of astrometric measurements are attributed to another source with 
Table 5. Orbital and Gaia DR1 details on the 256 planets analysed with GASTON.

\begin{tabular}{|c|c|c|c|c|c|c|c|c|c|c|c|c|c|c|c|c|c|}
\hline \multirow[b]{2}{*}{ Name } & \multicolumn{10}{|c|}{ RV data } & \multicolumn{7}{|c|}{ Gaia DR1 data } \\
\hline & $\begin{array}{c}P \\
\text { (days) }\end{array}$ & $\begin{array}{c}m \sin i \\
\left(M_{\mathrm{J}}\right)\end{array}$ & $\begin{array}{c}K \\
\left(\mathrm{~m} \mathrm{~s}^{-1}\right)\end{array}$ & $e$ & $\begin{array}{l}\omega \\
\left.{ }^{\circ}\right)\end{array}$ & $\begin{array}{c}T_{\mathrm{p}} \\
-2450000\end{array}$ & $\begin{array}{c}M_{\star} \\
\left(M_{\odot}\right)\end{array}$ & $\begin{array}{c}a_{\mathrm{p}} \\
(\mathrm{au})\end{array}$ & $\begin{array}{c}{ }_{\text {(mas) }}^{\pi} \\
\text { (n) }\end{array}$ & $\begin{array}{c}a_{\star} \sin i \\
\text { (mas) }\end{array}$ & $\begin{array}{c}\text { Transit } \\
\text { flag }\end{array}$ & $\begin{array}{l}\text { Drift } \\
\text { flag }\end{array}$ & $\begin{array}{l}{ }_{\text {DR1 }} \\
\text { (mas) }\end{array}$ & $N_{\mathrm{pts}}$ & $N_{\mathrm{FoV}}$ & $\begin{array}{c}\text { Gaia } \\
\text { dataset }\end{array}$ & $\begin{array}{c}\text { Duplicate } \\
\text { source }\end{array}$ \\
\hline 30 Ari B b & $335.1 \pm 2.5$ & $9.88 \pm 0.98$ & $272 \pm 24$ & $0.289 \pm 0.092$ & $307 \pm 18$ & $4538 \pm 20$ & $1.160 \pm 0.040$ & $0.992 \pm 0.012$ & $21.42 \pm 0.60$ & $0.173 \pm 0.019$ & $\mathrm{n}$ & $\mathrm{n}$ & 1.8 & 71 & 10 & 1 & $\mathrm{n}$ \\
\hline HAT-P-21 b & $4.1244810 \pm 0.0000070$ & $4.07 \pm 0.17$ & $548 \pm 14$ & $0.228 \pm 0.016$ & $309.0 \pm 3.0$ & $4995.014 \pm 0.046$ & $0.947 \pm 0.042$ & $0.04943 \pm 0.00073$ & $3.73 \pm 0.51$ & $0.00076 \pm 0.00011$ & $\mathrm{n}$ & $\mathrm{n}$ & 0.92 & 84 & 10 & 1 & $\mathrm{n}$ \\
\hline HD $114762 \mathrm{~b}$ & $83.9151 \pm 0.0030$ & $11.64 \pm 0.78$ & $612.5 \pm 3.5$ & $0.3354 \pm 0.0048$ & $201.3 \pm 1.0$ & $-110.89 \pm 0.19$ & $0.895 \pm 0.089$ & $0.361 \pm 0.012$ & $25.88 \pm 0.46$ & $0.116 \pm 0.015$ & $\mathrm{n}$ & $\mathrm{n}$ & 1.1 & 130 & 23 & 1 & $\mathrm{n}$ \\
\hline HD $132563 \mathrm{~B} \mathrm{~b}$ & $1544 \pm 34$ & $1.49 \pm 0.14$ & $26.7 \pm 2.2$ & $0.220 \pm 0.090$ & $158 \pm 35$ & $2593 \pm 148$ & $1.010 \pm 0.010$ & $2.623 \pm 0.039$ & $9.30 \pm 0.33$ & $0.0344 \pm 0.0034$ & $\mathrm{n}$ & $\mathrm{n}$ & 0.85 & 130 & 19 & 1 & $\mathrm{y}$ \\
\hline HD 141937 b & $653.2 \pm 1.2$ & $9.48 \pm 0.41$ & $234.5 \pm 6.4$ & $0.410 \pm 0.010$ & $187.72 \pm 0.80$ & $1847.4 \pm 2.0$ & $1.048 \pm 0.037$ & $1.497 \pm 0.018$ & $30.62 \pm 0.35$ & $0.396 \pm 0.023$ & $\mathrm{n}$ & $\mathrm{n}$ & 0.93 & 236 & 32 & 1 & $\mathrm{y}$ \\
\hline HD $148427 b$ & $331.5 \pm 3.0$ & $1.144 \pm 0.092$ & $27.7 \pm 2.0$ & $0.160 \pm 0.080$ & $277 \pm 68$ & $3991 \pm 15$ & $1.360 \pm 0.060$ & $1.039 \pm 0.017$ & $14.17 \pm 0.42$ & $0.0118 \pm 0.0012$ & $\mathrm{n}$ & $\mathrm{n}$ & 1.1 & 131 & 17 & 1 & $\mathrm{n}$ \\
\hline HD $154857 b$ & $408.60 \pm 0.50$ & $2.248 \pm 0.092$ & $48.3 \pm 1.0$ & $0.460 \pm 0.020$ & $57.0 \pm 4.0$ & $3572.5 \pm 2.4$ & $1.718 \pm 0.026$ & $1.2907 \pm 0.0066$ & $15.56 \pm 0.39$ & $0.0251 \pm 0.0013$ & $\mathrm{n}$ & $\mathrm{n}$ & 0.93 & 122 & 19 & 1 & $\mathrm{n}$ \\
\hline HD $154857 \mathrm{c}$ & $3452 \pm 105$ & $2.58 \pm 0.15$ & $24.2 \pm 1.1$ & $0.060 \pm 0.050$ & $352 \pm 37$ & $5219 \pm 375$ & $1.718 \pm 0.026$ & $5.35 \pm 0.11$ & $15.56 \pm 0.39$ & $0.1194 \pm 0.0081$ & $\mathrm{n}$ & $\mathrm{n}$ & 0.93 & 122 & 19 & 1 & $\mathrm{n}$ \\
\hline HD 164595 b & $40.00 \pm 0.24$ & $0.0508 \pm 0.0070$ & $3.05 \pm 0.41$ & $0.088 \pm 0.093$ & $145 \pm 135$ & $6280 \pm 12$ & $0.990 \pm 0.030$ & $0.2281 \pm 0.0025$ & $35.11 \pm 0.38$ & $0.000392 \pm 0.000056$ & $\mathrm{n}$ & $\mathrm{n}$ & 0.93 & 62 & 8 & 1 & $\mathrm{y}$ \\
\hline HD $16760 \mathrm{~b}$ & $465.1 \pm 2.3$ & $13.29 \pm 0.61$ & $408.0 \pm 7.0$ & $0.067 \pm 0.010$ & $232 \pm 10$ & $4723 \pm 12$ & $0.780 \pm 0.050$ & $1.081 \pm 0.023$ & 14 & 0.25 & $\mathrm{n}$ & $\mathrm{n}$ & 3.0 & 62 & 10 & 2 & $\mathrm{n}$ \\
\hline HD $177830 \mathrm{~b}$ & $410.1 \pm 2.2$ & $1.320 \pm 0.085$ & $32.64 \pm 0.98$ & $0.096 \pm 0.048$ & 189 & $254 \pm 49$ & $1.17 \pm 0.10$ & $1.138 \pm 0.033$ & $15.94 \pm 0.37$ & $0.0195 \pm 0.0022$ & $\mathrm{n}$ & $\mathrm{n}$ & 0.87 & 89 & 19 & 1 & $\mathrm{n}$ \\
\hline HD $185269 \mathrm{~b}$ & $6.8379 \pm 0.0010$ & $0.954 \pm 0.069$ & $90.7 \pm 4.4$ & $0.296 \pm 0.040$ & $172 \pm 11$ & $3154.089 \pm 0.040$ & $1.28 \pm 0.10$ & $0.0766 \pm 0.0020$ & $19.10 \pm 0.41$ & $0.00104 \pm 0.00012$ & $\mathrm{n}$ & $\mathrm{n}$ & 0.97 & 74 & 16 & 1 & $\mathrm{n}$ \\
\hline HD $190228 \mathrm{~b}$ & $1136.1 \pm 9.9$ & $5.94 \pm 0.30$ & $91.4 \pm 3.0$ & $0.531 \pm 0.028$ & $101.2 \pm 2.1$ & $3522 \pm 12$ & $1.821 \pm 0.046$ & $2.602 \pm 0.027$ & $15.77 \pm 0.34$ & $0.1278 \pm 0.0079$ & $\mathrm{n}$ & $\mathrm{n}$ & 0.86 & 116 & 16 & 1 & $\mathrm{y}$ \\
\hline HD 197037 b & $1036 \pm 13$ & $0.807 \pm 0.060$ & $15.5 \pm 1.0$ & $0.220 \pm 0.070$ & $298 \pm 26$ & $1353 \pm 86$ & 1.1 & 2.1 & $30.01 \pm 0.32$ & 0.043 & $\mathrm{n}$ & $\mathrm{n}$ & 0.99 & 107 & 17 & 1 & $\mathrm{n}$ \\
\hline HD $4203 b$ & $431.88 \pm 0.85$ & $2.08 \pm 0.12$ & $60.3 \pm 2.2$ & $0.519 \pm 0.027$ & $329.1 \pm 3.0$ & $1918.9 \pm 2.7$ & $1.130 \pm 0.064$ & $1.165 \pm 0.022$ & $12.67 \pm 0.44$ & $0.0259 \pm 0.0023$ & $\mathrm{n}$ & $\mathrm{n}$ & 0.85 & 60 & 11 & 1 & $\mathrm{n}$ \\
\hline HD $5388 \mathrm{~b}$ & $777.0 \pm 4.0$ & $1.97 \pm 0.10$ & $41.7 \pm 1.6$ & $0.400 \pm 0.020$ & $324.0 \pm 4.0$ & $4570.0 \pm 9.0$ & 1.2 & 1.8 & $18.86 \pm 0.32$ & 0.052 & $\mathrm{n}$ & $\mathrm{n}$ & 1.4 & 327 & 48 & 1 & $\mathrm{y}$ \\
\hline HD $6718 \mathrm{~b}$ & $2496 \pm 176$ & $1.56 \pm 0.12$ & $24.1 \pm 1.5$ & $0.100 \pm 0.075$ & $286 \pm 50$ & $4357 \pm 251$ & 0.96 & 3.6 & $19.74 \pm 0.41$ & 0.11 & $\mathrm{n}$ & $\mathrm{n}$ & 1.1 & 199 & 29 & 1 & $\mathrm{y}$ \\
\hline HD $7449 \mathrm{~b}$ & $1275 \pm 13$ & $1.31 \pm 0.52$ & $42 \pm 15$ & $0.820 \pm 0.060$ & $339.0 \pm 6.0$ & $5298 \pm 26$ & 1.1 & 2.3 & $27.14 \pm 0.41$ & 0.076 & $\mathrm{n}$ & $\mathrm{n}$ & 0.94 & 61 & 12 & 1 & $\mathrm{y}$ \\
\hline HD $95127 \mathrm{~b}$ & $482.0 \pm 5.0$ & $5.04 \pm 0.82$ & $116 \pm 12$ & $0.11 \pm 0.10$ & $40 \pm 38$ & $3200 \pm 50$ & $1.20 \pm 0.22$ & $1.278 \pm 0.079$ & $1.31 \pm 0.58$ & $0.0067 \pm 0.0034$ & $\mathrm{n}$ & $\mathrm{n}$ & 1.2 & 41 & 8 & 1 & $\mathrm{n}$ \\
\hline HD $96127 \mathrm{~b}$ & $647 \pm 17$ & $4.01 \pm 0.85$ & $105 \pm 11$ & $0.30 \pm 0.10$ & $162 \pm 18$ & $3969 \pm 31$ & $0.91 \pm 0.25$ & $1.42 \pm 0.13$ & $1.87 \pm 0.83$ & $0.0112 \pm 0.0064$ & $\mathrm{n}$ & $\mathrm{n}$ & 1.1 & 109 & 16 & 1 & $\mathrm{n}$ \\
\hline HIP $65891 \mathrm{~b}$ & $1084 \pm 23$ & $6.00 \pm 0.41$ & $64.9 \pm 2.4$ & $0.130 \pm 0.050$ & $356 \pm 16$ & $6015 \pm 49$ & $2.50 \pm 0.21$ & $2.804 \pm 0.088$ & $6.53 \pm 0.37$ & $0.0420 \pm 0.0053$ & $\mathrm{n}$ & $\mathrm{n}$ & 1.1 & 237 & 35 & 1 & $\mathrm{n}$ \\
\hline $\mathrm{K} 2-110 \mathrm{~b}$ & $13.86375 \pm 0.00026$ & $0.053 \pm 0.011$ & $5.5 \pm 1.1$ & $0.079 \pm 0.070$ & $90 \pm 122$ & 6863 & $0.738 \pm 0.018$ & $0.10207 \pm 0.00083$ & 8.6 & 0.000060 & $\mathrm{n}$ & $\mathrm{n}$ & 1.3 & 54 & 9 & 2 & $\mathrm{y}$ \\
\hline $\mathrm{K} 2-34 \mathrm{~b}$ & $2.9956290 \pm 0.0000060$ & $1.683 \pm 0.061$ & $207.0 \pm 3.0$ & $0.000 \pm 0.027$ & 90 & $7144.347030 \pm 0.000080$ & $1.226 \pm 0.052$ & $0.04353 \pm 0.00062$ & $3.28 \pm 0.68$ & $0.000187 \pm 0.000040$ & $\mathrm{n}$ & $\mathrm{n}$ & 1.00 & 117 & 16 & 1 & $\mathrm{y}$ \\
\hline WASP-11 b & $3.7224650 \pm 0.0000070$ & $0.540 \pm 0.052$ & $82.1 \pm 7.4$ & 0 & 90 & $4473.05588 \pm 0.00020$ & $0.800 \pm 0.025$ & $0.04364 \pm 0.00045$ & $7.49 \pm 0.58$ & $0.000211 \pm 0.000027$ & $\mathrm{n}$ & $\mathrm{n}$ & 1.1 & 60 & 10 & 1 & $\mathrm{n}$ \\
\hline WASP-131 b & $5.3220230 \pm 0.0000050$ & $0.272 \pm 0.018$ & $30.5 \pm 1.7$ & 0 & 90 & $6919.82360 \pm 0.00040$ & $1.060 \pm 0.060$ & $0.0608 \pm 0.0011$ & $4.55 \pm 0.56$ & $0.000068 \pm 0.000010$ & $\mathrm{n}$ & $\mathrm{n}$ & 0.95 & 53 & 7 & 1 & $\mathrm{n}$ \\
\hline WASP-156 b & $3.8361690 \pm 0.0000030$ & $0.1305 \pm 0.0087$ & $19.0 \pm 1.0$ & $0.0000 \pm 0.0035$ & 90 & $4677.7070 \pm 0.0020$ & $0.842 \pm 0.052$ & $0.04529 \pm 0.00093$ & 8.2 & 0.000055 & $\mathrm{n}$ & $\mathrm{n}$ & 1.5 & 38 & 7 & 2 & $\mathrm{n}$ \\
\hline WASP-157 b & $3.9516205 \pm 0.0000040$ & $0.559 \pm 0.049$ & $61.6 \pm 3.8$ & $0.000 \pm 0.055$ & 90 & $7257.803194 \pm 0.000088$ & $1.26 \pm 0.12$ & $0.0528 \pm 0.0017$ & $3.76 \pm 0.66$ & $0.000084 \pm 0.000019$ & $\mathrm{n}$ & $\mathrm{n}$ & 0.87 & 69 & 9 & 1 & $\mathrm{n}$ \\
\hline WASP-17 b & $3.7354330 \pm 0.0000076$ & $0.508 \pm 0.030$ & $59.2 \pm 2.9$ & 0 & 90 & $4559.18096 \pm 0.00023$ & $1.190 \pm 0.030$ & $0.04993 \pm 0.00042$ & $2.57 \pm 0.31$ & $0.0000523 \pm 0.0000072$ & $\mathrm{n}$ & $\mathrm{n}$ & 0.85 & 202 & 25 & 1 & $\mathrm{n}$ \\
\hline WASP-43 b & $0.8134750 \pm 0.0000010$ & $1.76 \pm 0.10$ & $550.3 \pm 6.7$ & 0 & 90 & $5528.86774 \pm 0.00014$ & $0.580 \pm 0.050$ & $0.01422 \pm 0.00041$ & 11 & 0.00047 & $\mathrm{n}$ & $\mathrm{n}$ & 2.0 & 63 & 7 & 2 & $\mathrm{n}$ \\
\hline
\end{tabular}

Notes. We only show here the 29 planets belonging to the "detection" sample, i.e. for which the astrometric excess noise surpasses the detection threshold (see Table 6). The full table, including the 227 planets from the "non-detection" sample, i.e. for which the astrometric excess noise is smaller than the threshold, is available at the CDS. Where uncertainties are missing we assume $10 \%$ errors on the corresponding parameter. The parallax with uncertainties are all taken from the DR1, while those without uncertainties are taken from SIMBAD. Orbit references can be found in the full-version of Table 2 at the CDS.

another ID, and thus lead to underestimate its AL angle residuals and its astrometric excess noise. This is of crucial importance if the mass of a companion is found to be smaller than $13.5 M_{\mathrm{J}}$. A larger astrometric excess noise leads to a larger mass range. We thus focus on the 227 non-duplicate companions orbiting 187 sources, which constitute the "non-detection sample".

The complete orbital and Gaia details of the 29 exoplanet candidates from the "detection sample" and analysed with GASTON are presented in Table 5. The complete table that also includes the 227 exoplanet candidates from the "non-detection sample" is available at the CDS. Table 6 summarizes all source selection steps applied from Sect. 2 up to the present section.

\section{GASTON simulations and new improvements}

\subsection{General principle}

In the present study, our goal is to constrain the inclination and true mass of RV planet candidates using the released Gaia astrometric data. To do so, we applied the GASTON method described in Kiefer et al. (2019) and Kiefer (2019). This algorithm simulates the residuals of Gaia's five-parameter fit of a source accounting for a supplementary astrometric motion due to a perturbing RV-detected companion. This leads to simulated astrometric excess noise $\varepsilon_{\text {simu }}$ depending on the actual inclination of the RV-detected orbital motion. The algorithm also accounts for measurement noise and modeling errors in the reduction of the DR1 through the noise model adopted in Sect. 3.4. These simulations are then compared to the astrometric excess noise measured by Gaia and reported in the DR1 database (Table 3) to derive a matching orbital inclination.

The GASTON algorithm is embedded into a MCMC process, with emcee (Foreman-Mackey et al. 2013), which allows us to derive the posterior distributions of orbital inclination and true mass of the RV companion among other parameters. To sum up, the varied physical parameters in the MCMC run are the orbital period $P$, eccentricity $e$, longitude of periastron $\omega$, periastron time of passage $T_{\mathrm{p}}$, inclination $I_{\mathrm{c}}$, minimum mass $m \sin i$, star mass $M_{\star}$, parallax $\pi$, hyper-parameter $f_{\varepsilon}$ to scale

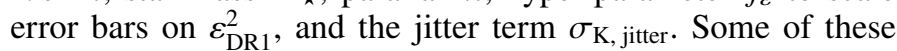
parameters have strong Gaussian priors from RV $\left(P, e, T_{\mathrm{p}}, \omega\right.$, $m \sin i$ ), or from other analysis $\left(M_{\star}, \pi\right)$. The hyper-parameter $f_{\varepsilon}$ follows a Gaussian prior about 0 with a standard deviation of 0.1 . The jitter term follows a flat prior between 0 and $\sqrt{3}$, assuming that the published uncertainty on $K$ could be underestimated by 
Table 6. Number of sources and planets in our sample after the several selection criteria introduced in Sects. 2, 3.2, and 3.5.

\begin{tabular}{|c|c|c|c|}
\hline Criterion & Article section & \# stellar hosts & \# planets \\
\hline exoplanets.org & 2 & 2466 & 3262 \\
\hline RV planets & 2 & 782 & 911 \\
\hline Sources in the DR1 & 3.1 & 658 & 755 \\
\hline$G>6.4$ & 3.2 & 614 & 705 \\
\hline$b-r<1.8$ & 3.2 & 580 & 654 \\
\hline \multicolumn{4}{|c|}{ Separation into primary/secondary Gaia DR1 datasets } \\
\hline Primary & 3.3 & 385 & 442 \\
\hline Secondary; $\log \left(\pi / \varepsilon_{\mathrm{DR} 1}\right)>0.5$ & $3.2 ; 3.3$ & 139 & 154 \\
\hline \multicolumn{4}{|c|}{$\varepsilon_{\mathrm{DR} 1}>$ threshold: the detection sample } \\
\hline Primary; $\varepsilon_{\mathrm{DR} 1}>0.85$ mas & 3.5 & 24 & 25 \\
\hline Secondary; $\varepsilon_{\mathrm{DR} 1}>1.2$ mas & 3.5 & 4 & 4 \\
\hline \multicolumn{4}{|c|}{$\varepsilon_{\mathrm{DR} 1}<$ threshold and non-transiting: the non-detection sample } \\
\hline Primary; $\varepsilon_{\mathrm{DR} 1}<0.85$ mas; no transit; non-duplicate & 3.5 & 165 & 201 \\
\hline Secondary; $\varepsilon_{\mathrm{DR} 1}<1.2$ mas; no transit; non-duplicate & 3.5 & 18 & 26 \\
\hline
\end{tabular}

as much as a factor $\sqrt{1+\sigma_{\mathrm{K}, \mathrm{jitter}}^{2}}=2$. Generally, we adopt a $\mathrm{d} p\left(I_{\mathrm{c}}\right)=\sin I_{\mathrm{c}} \mathrm{d} I_{\mathrm{c}}$ prior probability distribution for the inclination, assuming the inclination of orbits among RV candidates is isotropic. If the MCMC converges to an inclination strongly different from $90^{\circ}$ despite the low prior probability, this implies the data inputs have a significant weight in the likelihood.

\subsection{Dealing with proper motion and parallax in the simulations}

For sources in the primary dataset, we assume that the proper motion fit as performed by Gaia in the DR1 is disentangled from the hidden astrometric orbit. We thus assume that the astrometric excess noise is purely composed of noise and orbital motion and that it is not needed to fit out excess parallax and proper motion to the simulated astrometric orbit. This is justified by the addition of past HIPPARCOS or Tycho-2 positions in Gaia's reduction for fitting proper motion of primary dataset sources, thus based on astrometric measurements spanning more than $24 \mathrm{yr}$. Given that the orbital periods of all studied companions are smaller than $14 \mathrm{yr}$, the fit of proper motion to the simulated orbits reduces the amplitude of the simulated residuals - and thus of the astrometric excess noise - only by a small amount. Numerical simulations show that in the worst case scenario with a Tycho-2 position uncertainty of $\sim 100$ mas, the average simulated astrometric excess noise $\varepsilon_{\text {simu }}$ is lowered by less than 0.2 mas. This offset reduces to less than 0.05 mas if a HIPPARCOS position ( $\sigma_{\text {RA,Dec }} \sim 1$ mas) is used instead or if $P<10$ days. HIPPARCOS positions are available for 171 over the 190 primary sources in our sample, while only 6 sources have a Tycho- 2 position with more than 20 mas of uncertainty and a companion with $P>$ 10 days. These 6 sources, HD 95872, NGC 2423 3, BD+20 2457, HD 233604, BD+15 2375, and M67 SAND 364, all belong to the non-detection sample. For those, the astrometric excess noise that we simulate with GASTON for a given companion orbit and at a given orbital inclination could be overestimated by up to $\sim 0.2$ mas. Thus GASTON possibly underestimates the upper limit on the companion true mass for those stars.

For sources in the secondary dataset, the proper motion given in the DR1 is only derived from the Gaia data, without a supplementary data point from Tycho-2 or HIPPARCOS. An important part of the orbital motion could thus be mistaken for proper motion during the Gaia data reduction of the DR1, especially for orbital periods at which the Gaia measurements along the $416 \mathrm{~d}$ time baseline of the DR1 campaign could appear to be almost linear. For sources from the secondary dataset, we thus perform a fit of linear motion to the simulated astrometric orbit, from which residuals we derive $\varepsilon_{\text {simu }}$.

For sources of both datasets, fitting the parallax to the astrometric orbit does not have a significant effect on $\varepsilon_{\text {simu }}$ even if $P \sim 365 \mathrm{~d}$ and if the orbital and parallax motions are aligned. Numerical tests of parallax fit to simulated data along an astrometric orbit with $P \sim 365 \mathrm{~d}$ and randomizing along the unknown longitude of ascending node, $\Omega$ from 0 to $2 \pi$, leads to a typical reduction of the average $\varepsilon_{\text {simu }}$ smaller than 0.05 mas. Fitting parallax to the simulated astrometric orbit is thus unnecessary, leading to negligible deviations on the simulated astrometric excess noise.

\subsection{Recent improvements}

Since Kiefer (2019), we added the few following improvements. The number of walkers is reduced from 200 to 20, as it improves the speed of convergence of the MCMC while leading to equivalent results. The maximum number of iterations is increased to 1000000 . The MCMC stops whenever the autocorrelation length of every parameters stops, progressing by more than $1 \%$, and is at least 50 times smaller than the actual number of iterations. Finally, the host star and companion magnitude are calculated using a continuous series of models from planetary mass up to stellar mass of $30 M_{\odot}$. We also take into account the reflection of stellar light on the surface of the companion. These issues are discussed in Appendix A.

We highlight in this section an important effect of the modeling of the light reflected from the companion surface on the motion of the photocenter, which is developed in more detail in Appendix A. For mass ratios $M_{\mathrm{c}} / M_{\star} \sim 10^{-5}-10^{-3}$ and companion orbit semimajor axis $a_{\mathrm{c}}<0.5$ a.u., the companion reflected light can become more important than the emission of a star in the calculation of the photocenter semimajor axis, where $a_{\mathrm{ph}}=$ $L_{\mathrm{c}} a_{\mathrm{c}}+L_{\star} a_{\star}$. The astrometric motion of the system observed from Earth can even follow the motion of the companion itself rather than the motion of the stellar host. This could lead to 
wrongly determine the orientation (retrograde/prograde) of the primary star orbit, and strongly underestimate the primary star semimajor axis and thus the mass of the companion. This effect cannot be seen in the present study because it is smaller than the adopted detection thresholds (Sect. 3.5), but should be taken into account in future analysis of Gaia's time series of systems with planets.

The definition of the parameters explored in the MCMC corresponding to inclination, eccentricity, and longitude $\omega$ are improved compared to Kiefer (2019), solving singularity issues at the border of the domain expected for these parameters. Adopting $\lambda_{I_{\mathrm{c}}}=\tan \left(2 I_{\mathrm{c}}-\pi / 2\right)$ as was used in Kiefer (2019) led the StretchMove algorithm of emcee (Foreman-Mackey et al. 2013) to get stuck in low probability regions with large or small inclinations, much wider in terms of $\lambda_{I_{\mathrm{c}}}$. We thus consider instead simply varying $I_{\mathrm{c}}$ imposing rigid boundaries at $I_{\mathrm{c}}=0$ and $\pi / 2$. Moreover, the exploration of the $(e, \omega)$ space was not optimal, especially around the singularity $e=0$. We now vary instead $\lambda_{\mathrm{c}}=\tan \left(\frac{\pi}{2} e \cos \omega\right)$ and $\lambda_{\mathrm{s}}=\tan \left(\frac{\pi}{2} e \sin \omega\right)$, where $e$ and $\omega$ are then obtained from the simple transformations and combinations of $\lambda_{\mathrm{c}}$ and $\lambda_{\mathrm{s}}$.

\subsection{Application of GASTON to the defined samples}

We applied GASTON on the 29 candidate exoplanets of the detection sample, orbiting the 28 sources whose astrometric excess noise exceed the detection thresholds fixed in Sect. 3.5 and listed in Table 5. For those sources, with the orbit of the star a priori detected in the astrometric data, an inclination and true mass could technically be measured.

For the 227 non-transiting companions of the non-detection sample listed in full Table 5 (available at the CDS), we also used GASTON to derive the lowest inclination and largest mass possible for the companion, beyond which the astrometric excess noise would become too large to be compatible with $\varepsilon_{\mathrm{DR} 1}$. To limit the computation time, and since these calculations only lead to parameter ranges and not strict measurements, we reduced the maximum number of MCMC steps in GASTON to 50000 for these 227 companions. Moreover, for those 227 companions we adopted a flat prior for the inclination, conversely to that adopted for the detection sample. The shape of the prior distribution of the inclination tends to dictate the shape of the posterior distributions if the simulated astrometric excess noise is compatible with $\varepsilon_{\mathrm{DR} 1}$ for inclinations of $90^{c} \mathrm{irc}$ down to $\sim 0^{\circ}$. This prior artificially increases the conventional lower limit, such as $3 \sigma$, for inclination, and thus decreases the upper limit on mass. This is typically the case for companions in the non-detection sample with $\varepsilon_{\mathrm{DR} 1}$ compatible with noise and $a_{\star} \sin I \ll \varepsilon_{\mathrm{DR} 1}$. Adopting a flat prior for the inclination instead favors the likelihood - and thus the data - to dictate the shape of the posterior distributions down to a small inclination. This better reveals the variations of the inclination and mass posteriors only due to incompatibilities between the $\varepsilon_{\mathrm{simu}}$ and $\varepsilon_{\mathrm{DR} 1}$ at inclinations close to $0^{\circ}$.

In the following, we only report for the resulting posteriors of the inclination $I_{\mathrm{c}}$ and its deriving parameters: the true mass of the companion, the photocenter semimajor axis, and the magnitude difference between the companion and its host star.

\section{Results}

\subsection{General results}

We identified the following three possible situations from the results produced by GASTON:
Table 7. Two exoplanets, WASP-43 b and WASP-156b, whose RV orbits are incompatible with Gaia astrometric excess noise.

\begin{tabular}{lccc}
\hline \hline Parameter & Unit & WASP-43 b & WASP-156 b \\
\hline Period & $($ day $)$ & 0.813 & 3.836 \\
$m \sin i$ & $\left(M_{\mathrm{J}}\right)$ & 1.761 & 0.131 \\
$a \sin i$ & $(\mathrm{mas})$ & 0.00047 & 0.000055 \\
$\varepsilon_{\mathrm{DR} 1}$ & $(\mathrm{mas})$ & 1.96 & 1.49 \\
$\varepsilon_{\text {simu,max }}$ & $($ mas $)$ & 1.25 & 1.32 \\
\hline
\end{tabular}

Notes. The maximum astrometric excess noise that we were able to simulate for these secondary dataset sources is given as $\varepsilon_{\text {simu,max }}$.

1. Orbits leading to a firm measurement of the RV orbit inclination and the true mass of the companions. This concerns 9 exoplanet candidates out of 29 in the detection sample. This is summarized in Sect. 5.2.1;

2. Orbits for which the astrometry cannot constrain the inclination. Because of the noise, producing a measured astrometric excess noise that is compatible with the RV orbital motion is possible for a large range of inclinations. The derived solution mainly follows the sin $i$ prior distribution of inclination, with a median about $60^{\circ}, 1 \sigma$ confidence interval within 30 $80^{\circ}$, and a $3 \sigma(99.85 \%)$ percentile larger than $89.5^{\circ}$. Only the upper limit on the mass and the lower limit on the inclination is informative. This concerns 18 exoplanet candidates from the detection sample and the 227 exoplanet candidates from the non-detection sample. This is summarized in Sects. 5.2.2 and 5.3;

3. Companions for which the astrometric excess noise could never be reached in the simulations testing any inclinations from 0.001 to $90^{\circ}$. The Gaia astrometric excess noise is incompatible with the published RV orbit. Two companions from the detection sample, WASP-43 b and WASP-156 b, are in this situation (see Sect. 5.2.3).

For the 29 companions of the detection sample, the results of GASTON according to different situations introduced above are presented in Tables 7 and 8. Moreover, Table 9, the full-version of which is available at the CDS, summarizes the parameter limits derived for the 227 companions of the non-detection sample. In both tables, we list the resulting corrected mass, astrometric orbit semimajor axis, estimated magnitude difference between the host and the companion, MCMC acceptance rate, and convergence indicator $N_{\text {steps }} / \max \left(\tau_{\lambda}\right)$ (see below).

The acceptance rate delivered by emcee allows us to quantify the probability of reaching $\varepsilon_{\mathrm{DR} 1}$ through all simulations performed during the MCMC process. Typically, if an MCMC performs well, the acceptance rate must reach $0.2-0.4$. This is the case for all nine companions entering situation \#1, except one, HD $96127 \mathrm{~b}$ for which the acceptance rate is 0.06 . Low values of the acceptance rate usually imply overly large steps in the Monte Carlo process (Foreman-Mackey et al. 2013). We can firmly exclude any "steps issue", since the geometry of the parameter space is the same for all systems, and the steps for the different parameters have been tuned such that well-behaved cases fulfil the $0.2-0.4$ criterion. Rather we explain this low acceptance rate by the presence of noise in our simulations. A fortuitous pileup of noise can allow some simulations to be compatible with $\varepsilon_{\mathrm{DR} 1}=1.124$ mas even with an inclination close to $90^{\circ}$ and a negligible photocenter orbit. With a sin $i$-prior on inclination favoring the edge-on configuration, this is sufficient to drag the MCMC toward exploring regions where producing such astrometric excess noise is not frequent. The low acceptance 
Table 8. GASTON results for the 29 companions in the detection sample divided into the two different situations mentioned in the text.

\begin{tabular}{|c|c|c|c|c|c|c|c|c|c|c|c|c|c|}
\hline \multirow[t]{3}{*}{ Planet name } & \multirow{3}{*}{$\begin{array}{l}\text { Period } \\
\text { (days) }\end{array}$} & \multirow{3}{*}{$\begin{array}{c}m \sin i \\
\left(M_{\mathrm{J}}\right)\end{array}$} & \multirow{3}{*}{$\begin{array}{l}a \sin i \\
(\mathrm{mas})\end{array}$} & \multirow{3}{*}{$\begin{array}{c}\epsilon \\
\text { (mas) }\end{array}$} & \multicolumn{2}{|c|}{$a_{\text {phot }}$} & \multirow{2}{*}{\multicolumn{2}{|c|}{$\begin{array}{l}I_{\mathrm{c}} \\
\left({ }^{\circ}\right) \\
\end{array}$}} & \multirow{2}{*}{\multicolumn{2}{|c|}{$\begin{array}{c}M_{\mathrm{c}, \text { true }} \\
\left(M_{\mathrm{J}}\right) \\
\end{array}$}} & \multicolumn{2}{|c|}{$\Delta V$} & \multirow{3}{*}{$\begin{array}{c}\text { MCMC } \\
\text { Acceptance } \\
\text { rate }\end{array}$} \\
\hline & & & & & (mas) & (mas) & & & & & & & \\
\hline & & & & & $1 \sigma$ & $3 \sigma$ & $1 \sigma$ & $3 \sigma$ & $1 \sigma$ & $3 \sigma$ & $1 \sigma$ & $3 \sigma$ & \\
\hline
\end{tabular}

First situation: Strong constraint on inclination and mass

\begin{tabular}{|c|c|c|c|c|c|c|c|c|c|c|c|c|c|}
\hline \multicolumn{14}{|l|}{ Primary dataset: } \\
\hline 30 Ari B b & 335.1 & 9.878 & 0.1728 & 1.777 & $2.391_{-0.444}^{+0.592}$ & $(1.347,5.948)$ & $4.181_{-0.931}^{+1.031}$ & $(1.643,7.584)$ & $147.4_{-29.5}^{+41.3}$ & $(78.92,412.5)$ & $11.14_{-1.47}^{+1.05}$ & $(6.888,16.46)$ & 0.2321 \\
\hline HD 114762 b & 83.92 & 11.64 & 0.1161 & 1.088 & $1.339_{-0.353}^{+0.302}$ & $(0.4206,2.597)$ & $4.940_{-0.942}^{+1.773}$ & $(2.517,15.76)$ & $147.0_{-42.0}^{+39.3}$ & $(41.93,323.9)$ & $9.350_{-1.413}^{+1.614}$ & $(5.704,22.37)$ & 0.1758 \\
\hline HD 141937 b & 653.2 & 9.475 & 0.3955 & 0.9337 & $1.126_{-0.401}^{+0.272}$ & $(0.3798,2.047)$ & $20.52_{-4.16}^{+12.47}$ & $(11.01,88.85)$ & $27.42_{-9.86}^{+6.78}$ & $(9.061,50.94)$ & $24.38_{-0.61}^{+1.29}$ & $(22.82,29.59)$ & 0.1340 \\
\hline HD 148427 b & 331.5 & 1.144 & 0.01182 & 1.092 & $1.329_{-0.310}^{+0.332}$ & $(0.4964,2.936)$ & $0.5120_{-0.1082}^{+0.1635}$ & $(0.2314,1.413)$ & $136.5_{-33.7}^{+37.2}$ & $(47.74,336.0)$ & $12.34_{-1.32}^{+1.26}$ & $(8.368,24.50)$ & 0.1866 \\
\hline HD 5388 b & 777.0 & 1.965 & 0.05154 & 1.365 & $2.182_{-0.255}^{+0.330}$ & $(1.519,3.923)$ & $1.356_{-0.191}^{+0.195}$ & $(0.7610,2.050)$ & $87.02_{-10.80}^{+13.99}$ & $(60.02,163.3)$ & $14.95_{-1.82}^{+4.58}$ & $(10.81,23.13)$ & 0.2394 \\
\hline HD 6718 b & 2496 & 1.559 & 0.1087 & 1.121 & $4.201_{-0.881}^{+1.092}$ & $(1.982,9.951)$ & $1.488_{-0.310}^{+0.410}$ & $(0.6181,3.688)$ & $62.79_{-13.80}^{+16.98}$ & $(27.74,159.7)$ & $21.22_{-5.60}^{+1.20}$ & $(9.348,24.13)$ & 0.2073 \\
\hline HD $96127 b^{(\dagger)}$ & 647.3 & 4.007 & 0.01116 & 1.124 & $0.6640_{-0.6457}^{+0.7888}$ & $(0.0009983,2.231)$ & $1.364_{-0.763}^{+38.527}$ & $(0.4084,89.54)$ & $190.2_{-184.0}^{+284.1}$ & $(3.359,679.2)$ & $8.008_{-3.522}^{+16.829}$ & $(2.526,26.81)$ & 0.05775 \\
\hline HIP 65891 b & 1084 & 6.001 & 0.04197 & 1.146 & $2.040_{-0.341}^{+0.409}$ & $(1.103,4.026)$ & $1.184_{-0.207}^{+0.256}$ & $(0.5929,2.161)$ & $312.3_{-57.4}^{+74.2}$ & $(168.4,713.7)$ & $10.85_{-0.63}^{+0.65}$ & $(7.196,13.52)$ & 0.2000 \\
\hline \multicolumn{14}{|c|}{ Secondary dataset } \\
\hline HD 16760 b & 465.1 & 13.29 & 0.2531 & 2.990 & $4.467_{-0.758}^{+1.053}$ & $(2.758,7.364)$ & $3.164_{-0.762}^{+0.810}$ & $(1.796,5.549)$ & $291.9_{-69.4}^{+120.7}$ & $(151.8,580.9)$ & $5.154_{-1.124}^{+0.926}$ & $(2.517,8.215)$ & 0.2521 \\
\hline
\end{tabular}

\begin{tabular}{|c|c|c|c|c|c|c|c|c|c|c|c|c|c|}
\hline \multicolumn{14}{|c|}{ Second situation: lower and upper limits on inclination and mass } \\
\hline \multicolumn{14}{|l|}{ Primary dataset: } \\
\hline HAT-P-21 b & 4.124 & 4.073 & 0.0007560 & 0.9171 & $<0.0014260$ & $<0.01209$ & $>32.88$ & $>4.067$ & $<7.542$ & $<57.72$ & $>14.79$ & $>13.58$ & 0.009911 \\
\hline HD 132563 B b & 1544 & 1.492 & 0.03442 & 0.8536 & $<0.07070$ & $<2.621$ & $>29.25$ & $>0.8231$ & $<3.050$ & $<111.1$ & $>25.10$ & $>11.03$ & 0.01422 \\
\hline HD 154857 b & 408.6 & 2.248 & 0.02508 & 0.9309 & $<0.05492$ & $<1.499$ & $>27.03$ & $>1.014$ & $<4.918$ & $<134.9$ & $>24.71$ & $>13.10$ & 0.009493 \\
\hline HD $154857 \mathrm{c}$ & 3452 & 2.579 & 0.1193 & 0.9309 & $<0.2729$ & $<8.817$ & $>25.98$ & $>0.9194$ & $<5.905$ & $<175.3$ & $>27.78$ & $>10.97$ & 0.009493 \\
\hline HD 164595 b & 40.00 & 0.05078 & 0.0003920 & 0.9341 & $<0.0008560$ & $<0.1093$ & $>27.67$ & $>0.2476$ & $<0.11103$ & $<12.86$ & $>24.46$ & $>22.70$ & 0.01065 \\
\hline HD $177830 \mathrm{~b}$ & 410.1 & 1.320 & 0.01953 & 0.8723 & $<0.04035$ & $<1.278$ & $>28.82$ & $>1.019$ & $<2.738$ & $<78.92$ & $>24.47$ & $>13.43$ & 0.01443 \\
\hline HD 185269 b & 6.838 & 0.9542 & 0.001040 & 0.9694 & $<0.002002$ & $<0.01755$ & $>31.18$ & $>4.624$ & $<1.820$ & $<12.34$ & $>19.00$ & $>18.87$ & 0.005830 \\
\hline HD 190228 b & 1136 & 5.942 & 0.1278 & 0.8628 & $<0.5136$ & $<2.375$ & $>14.25$ & $>3.188$ & $<24.418$ & $<111.4$ & $>27.35$ & $>14.34$ & 0.02329 \\
\hline HD 197037 b & 1036 & 0.8073 & 0.04322 & 0.9947 & $<0.12112$ & $<3.087$ & $>21.09$ & $>0.8682$ & $<2.2696$ & $<55.87$ & $>27.13$ & $>22.28$ & 0.008977 \\
\hline HD $4203 \mathrm{~b}$ & 431.9 & 2.082 & 0.02595 & 0.8539 & $<0.05646$ & $<1.390$ & $>27.46$ & $>1.116$ & $<4.533$ & $<110.2$ & $>24.02$ & $>11.82$ & 0.02863 \\
\hline HD 7449 b & 1275 & 1.313 & 0.07578 & 0.9430 & $<0.16248$ & $<6.525$ & $>30.30$ & $>0.8955$ & $<2.845$ & $<104.2$ & $>27.17$ & $>11.48$ & 0.01279 \\
\hline HD $95127 b^{(\dagger)}$ & 482.0 & 5.036 & 0.006734 & 1.220 & $<0.017628$ & $<0.3977$ & $>26.57$ & $>2.027$ & $<11.863$ & $<170.2$ & $>23.04$ & $>6.939$ & 0.001910 \\
\hline $\mathrm{K} 2-34 \mathrm{~b}^{(\dagger)}$ & 2.996 & 1.683 & 0.0001870 & 0.9982 & $<0.0004070$ & $<0.002572$ & $>28.51$ & $>3.946$ & $<3.525$ & $<23.91$ & $>13.63$ & $>12.40$ & 0.002133 \\
\hline WASP-11 b & 3.722 & 0.5398 & 0.0002100 & 1.064 & $<0.0004260$ & $<0.004463$ & $>29.21$ & $>3.117$ & $<1.1059$ & $<9.719$ & $>15.62$ & $>15.27$ & 0.003389 \\
\hline WASP-131 b & 5.322 & 0.2724 & 0.00006800 & 0.9476 & $<0.00014300$ & $<0.001557$ & $>28.30$ & $>4.120$ & $<0.5756$ & $<3.710$ & $>15.31$ & $>14.62$ & 0.01006 \\
\hline WASP-157 b & 3.952 & 0.5592 & 0.00008400 & 0.8699 & $<0.0001670$ & $<0.001673$ & $>30.85$ & $>3.765$ & $<1.1127$ & $<8.444$ & $>14.31$ & $>13.06$ & 0.01517 \\
\hline WASP-17 b & 3.735 & 0.5077 & 0.00005200 & 0.8509 & $<0.00010000$ & $<0.0007792$ & $>32.14$ & $>5.985$ & $<0.9619$ & $<4.768$ & $>13.48$ & $>12.70$ & 0.006831 \\
\hline \multicolumn{14}{|c|}{ Secondary dataset: } \\
\hline $\mathrm{K} 2-110 \mathrm{~b}^{(\stackrel{\star}{)})}$ & 13.86 & 0.05293 & 0.00006000 & 1.278 & $<0.00069800$ & $<0.01073$ & $>4.96$ & $>0.3551$ & $<0.62324$ & $<6.732$ & $>18.03$ & $>17.49$ & 0.0007182 \\
\hline
\end{tabular}

Notes. ${ }^{(\dagger)}$ After 1000000 iterations MCMC did not reach convergence, with a final maximum autocorrelation length larger than $N_{\text {step }} / 50$.

rate is a reflection of this low frequency. This leads, in the case of HD $96127 \mathrm{~b}$, to a $3 \sigma$ upper limit on the inclination of $89.54^{\circ}$. This is the same mechanism that explains the small acceptance rates associated with mass upper limits for all companions entering situation \#2.

The autocorrelation length $\tau_{\lambda}$ probes the quality of a $\lambda$ parameter exploration by the MCMC during a run. With emcee and its Goodman-Weare algorithm (Goodman \& Weare 2010) it can be considered that convergence is reached if at least $N_{\text {steps }} / \tau_{\lambda}>50$ (Foreman-Mackey et al. 2013), and at best if $\delta \tau_{\lambda} / \tau_{\lambda}<1 \%$ for all parameters $\lambda$. The errors on the estimations of the posteriors are then reduced by a factor smaller than $1 / \sqrt{50} \sim 0.14$. Longer chains obviously produce more accurate results, but are also more time consuming. In this paper, we do not aim for perfect accuracy because our study is only based on a preliminary estimation of one quantity, the astrometric excess noise, by Gaia. We thus decided to stop the MCMC whenever $N_{\text {steps,max }}$ is reached or $N_{\text {steps }} / \max \left(\tau_{\lambda}\right)>50$ and $\delta \tau_{\lambda} / \tau_{\lambda}<1 \%$ for all parameters $\lambda$. With up to 1000000 steps and 20 walkers for 10 parameters to explore, the MCMC should have enough time to converge. This allows us to identify problematic systems, such as HD $96127 \mathrm{~b}$, for which the exploration of the parameter space is inefficient. In Table 8, we identify three companions, including HD $96127 \mathrm{~b}$, for which GASTON did not converge after $N_{\text {step }}=1000000$ iterations, with a maximum autocorrelation length larger than $N_{\text {step }} / 50$ and a small acceptance rate. The posteriors for those companions cannot be reliable, and the width of the confidence intervals on their mass is most likely underestimated.

\subsection{Detection sample}

\subsubsection{Situation \#1: Mass measurement for two possible massive exoplanets, two BDs and five $\mathrm{M}$ dwarfs}

We illustrates this first case scenario in Fig. 7 with the example of 30 Ari B b (Guenther et al. 2009), for which with a period of 335 days, the astrometric excess noise of 1.78 mas leads to 
F. Kiefer et al.: Determining the true mass of radial-velocity exoplanets with Gaia

Table 9. GASTON results obtained for the exoplanet candidates belonging to the non-detection sample.

\begin{tabular}{|c|c|c|c|c|c|c|c|c|c|}
\hline Planet name & $\begin{array}{c}\text { Period } \\
\text { (days) }\end{array}$ & $\begin{array}{c}m \sin i \\
\left(M_{\mathrm{J}}\right)\end{array}$ & $\begin{array}{c}a \sin i \\
(\mathrm{mas})\end{array}$ & $\begin{array}{c}\epsilon \\
\text { (mas) }\end{array}$ & $\begin{array}{c}a_{\text {phot }} \\
\text { (mas) } \\
3 \sigma\end{array}$ & $\begin{array}{l}I_{\mathrm{c}} \\
\left(^{\circ}\right) \\
3 \sigma\end{array}$ & $\begin{array}{c}M_{\mathrm{c}, \text { true }} \\
\left(M_{\mathrm{J}}\right) \\
3 \sigma\end{array}$ & $\begin{array}{l}\Delta V \\
3 \sigma\end{array}$ & $\begin{array}{c}\text { MCMC } \\
\text { acceptance } \\
\text { rate }\end{array}$ \\
\hline \multicolumn{10}{|c|}{ 3- $\sigma$ limits, with $M_{\mathrm{c}, \text { true }}<13.5 M_{\mathrm{J}}$ at $3 \sigma$} \\
\hline \multicolumn{10}{|c|}{ Primary dataset: 24 confirmed exoplanets } \\
\hline $\mathrm{BD}-061339 \mathrm{~b}$ & 3.873 & 0.02680 & 0.00007800 & 0.5190 & $<0.03085$ & $>0.3243$ & $<4.792$ & $>19.77$ & 0.1357 \\
\hline $\mathrm{BD}-082823 \mathrm{~b}$ & 5.600 & 0.04594 & 0.00008000 & 0.4011 & $<0.02921$ & $>0.2746$ & $<9.277$ & $>18.80$ & 0.1914 \\
\hline HD $10180 \mathrm{c}$ & 5.760 & 0.04151 & 0.00006200 & 0.4662 & $<0.01925$ & $>0.2841$ & $<8.626$ & $>19.26$ & 0.1585 \\
\hline HD $10180 \mathrm{~d}$ & 16.36 & 0.03766 & 0.0001120 & 0.4662 & $<0.06159$ & $>0.2005$ & $<10.37$ & $>20.77$ & 0.1592 \\
\hline HD $10180 \mathrm{~g}$ & 602.0 & 0.06738 & 0.002221 & 0.4662 & $<0.4390$ & $>0.3663$ & $<10.62$ & $>25.92$ & 0.1496 \\
\hline HD $125595 \mathrm{~b}$ & 9.674 & 0.04168 & 0.0001510 & 0.4106 & $<0.08048$ & $>0.2243$ & $<11.11$ & $>20.48$ & 0.2058 \\
\hline HD $154345 b$ & 3342 & 0.9569 & 0.2360 & 0.3491 & $<3.454$ & $>4.652$ & $<11.94$ & $>26.02$ & 0.1737 \\
\hline HD $175607 \mathrm{~b}$ & 29.03 & 0.02626 & 0.0001440 & 0.4171 & $<0.07016$ & $>0.1865$ & $<7.728$ & $>21.26$ & 0.1692 \\
\hline HD 176986 b & 6.490 & 0.02002 & 0.00005500 & 0.2559 & $<0.02388$ & $>0.2581$ & $<4.681$ & $>19.98$ & 0.1911 \\
\hline HD $176986 \mathrm{c}$ & 16.82 & 0.02814 & 0.0001450 & 0.2559 & $<0.05738$ & $>0.2419$ & $<6.601$ & $>21.35$ & 0.1939 \\
\hline HD 179079 b & 14.48 & 0.08378 & 0.0001240 & 0.3794 & $<0.03729$ & $>0.3766$ & $<13.20$ & $>19.21$ & 0.1853 \\
\hline HD 181433 b & 9.374 & 0.02373 & 0.00008600 & 0.2972 & $<0.04037$ & $>0.2542$ & $<5.376$ & $>20.49$ & 0.1210 \\
\hline HD $181433 \mathrm{c}$ & 962.0 & 0.6404 & 0.05114 & 0.2972 & $<0.6246$ & $>5.196$ & $<6.944$ & $>27.15$ & 0.1185 \\
\hline HD $181433 d$ & 2172 & 0.5355 & 0.07359 & 0.2972 & $<1.747$ & $>2.665$ & $<11.28$ & $>25.41$ & 0.1186 \\
\hline HD $215152 \mathrm{~b}$ & 5.760 & 0.005720 & 0.00001900 & 0.3057 & $<0.009679$ & $>0.1871$ & $<1.779$ & $>20.36$ & 0.1786 \\
\hline HD 215152 c & 7.282 & 0.005408 & 0.00002100 & 0.3057 & $<0.01000$ & $>0.2035$ & $<1.475$ & $>20.70$ & 0.1799 \\
\hline HD $215152 d$ & 10.86 & 0.008816 & 0.00004400 & 0.3057 & $<0.02144$ & $>0.1998$ & $<2.424$ & $>21.27$ & 0.1783 \\
\hline HD $215152 \mathrm{e}$ & 25.20 & 0.009052 & 0.00008000 & 0.3057 & $<0.04964$ & $>0.1869$ & $<3.069$ & $>22.48$ & 0.1794 \\
\hline HD $215497 \mathrm{~b}$ & 3.934 & 0.02085 & 0.00002600 & 0.4831 & $<0.01194$ & $>0.2301$ & $<4.999$ & $>18.50$ & 0.1164 \\
\hline HD 7199 b & 615.0 & 0.2950 & 0.01192 & 0.3742 & $<0.5161$ & $>1.496$ & $<11.45$ & $>25.73$ & 0.1817 \\
\hline HD 7924 b & 5.398 & 0.02737 & 0.0001050 & 0.5727 & $<0.04275$ & $>0.2472$ & $<6.476$ & $>20.81$ & 0.1021 \\
\hline HD $7924 \mathrm{c}$ & 15.30 & 0.02484 & 0.0001900 & 0.5727 & $<0.08133$ & $>0.2575$ & $<5.546$ & $>22.31$ & 0.1015 \\
\hline HD $7924 \mathrm{~d}$ & 24.45 & 0.02038 & 0.0002130 & 0.5727 & $<0.1022$ & $>0.2188$ & $<4.934$ & $>22.99$ & 0.1091 \\
\hline HIP 57274 b & 8.135 & 0.03657 & 0.0001300 & 0.4507 & $<0.06026$ & $>0.1980$ & $<10.57$ & $>20.32$ & 0.1738 \\
\hline \multicolumn{10}{|c|}{ Secondary dataset: 3 confirmed exoplanets } \\
\hline HD $40307 \mathrm{~b}$ & 4.311 & 0.01291 & 0.00006000 & 0.3337 & $<0.03068$ & $>0.2022$ & $<3.741$ & $>20.72$ & 0.1891 \\
\hline HD $40307 \mathrm{c}$ & 9.620 & 0.02115 & 0.0001690 & 0.3337 & $<0.07895$ & $>0.2109$ & $<5.879$ & $>21.69$ & 0.1899 \\
\hline HD $40307 \mathrm{~d}$ & 20.46 & 0.02808 & 0.0003710 & 0.3337 & $<0.1125$ & $>0.2855$ & $<5.586$ & $>22.81$ & 0.1927 \\
\hline
\end{tabular}

Notes. We only show here the 27 companions confirmed as planets, with $M_{\max , 3 \sigma}<13.5 M_{\mathrm{J}}$. The full table, including the rest of the exoplanet candidades among the non-detection sample and for which $M_{\max , 3 \sigma}>13.5 M_{\mathrm{J}}$, is available at the CDS.

an inclination of $4.14_{-0.90}^{\circ+0.96}$ and a corrected mass of $148_{-27}^{+42} M_{\mathrm{J}}$ instead of an $m \sin i=10 \pm 1 M_{\mathrm{J}}$. The top panel of Fig. 7 shows the simulated astrometric excess noise obtained for 10000 different values of inclinations from 0.001 to $90^{\circ}$. For any inclinations below $1^{\circ}$, the true mass of $30 \mathrm{AriB} \mathrm{b}$ is too large and the magnitude difference with the primary star is smaller than 2.5 ; these simulations are ignored since they would imply the presence of a detectable secondary component in the spectrum of this system, conversely to that observed. The bottom panel of Fig. 7 compares the $I_{\mathrm{c}}$ posterior distribution PDF to the PDF of an ensemble of same size drawn from the assumed prior density function, $\mathrm{d} p=\sin I_{\mathrm{c}} \mathrm{d} I_{\mathrm{c}}$. This posterior PDF is very distinct from the prior $\mathrm{PDF}$, which thus has a minor impact on the posterior distributions output from the MCMC. The corner plot of all posterior distributions for 30 Ari B b is shown in Fig. 8.

In this category, all other GASTON runs work similar to that of 30 Ari B b, with the exception of HD $96127 \mathrm{~b}$, whose MCMC run could not converge after 1000000 iterations. In total, the true masses for nine exoplanet candidates could be determined using GASTON, with eight orbiting sources from the primary dataset and one, HD $16760 \mathrm{~b}$, from the secondary dataset. We determined that seven of the companions are not planets and that two could likely be BDs or M dwarfs, but the planetary nature cannot be excluded at $3 \sigma$.

Among the primary sources, we find that HD $5388 \mathrm{~b}$ (Santos et al. 2010), HD 6718 b (Naef et al. 2010), HD 114762 b (Latham et al. 1989; Kane et al. 2011), and HD 148427 b (Fischer et al. 2009) are constrained within the BD to M-dwarf domain with the $3 \sigma$ mass ranges $(57,150) M_{\mathrm{J}},(29,157) M_{\mathrm{J}},(33,328) M_{\mathrm{J}}$, and $(27,345) M_{\mathrm{J}}$, respectively. We found moreover that $30 \mathrm{Ari} \mathrm{B} \mathrm{b}$ and HIP $65891 \mathrm{~b}$ (Jones et al. 2015) are stars in the M-dwarf mass regime with masses larger than $80 M_{\mathrm{J}}$.

The two possible planets are HD $141937 \mathrm{~b}$ (Udry et al. 2002) and HD 96127 b (Gettel et al. 2012). The true mass of HD $141937 \mathrm{~b}$ is located just beyond the boundary between massive planets and low-mass BDs with $M=27.5_{-10.8}^{+6.9} M_{\mathrm{J}}$ at $1 \sigma$, but the mass of this candidate is possibly as low as $9 M_{\mathrm{J}}$ at $3 \sigma$.

The true mass of HD $96127 \mathrm{~b}$ is most likely well within the stellar domain with $M=190_{-184}^{+284} M_{\mathrm{J}}$ and an inclination $I_{\mathrm{c}}=$ $1.364_{-0.763}^{+38.527}$ at $1 \sigma$. Within the $1-\sigma$ confidence interval, a true mass of HD $96127 \mathrm{~b}$ as low as $6 M_{\mathrm{J}}$ could also be compatible with $\varepsilon_{\mathrm{DR} 1}$. However, we already noted that GASTON did not converge for this precise case owing to a marginal, but possible, compatibility of the Gaia DR1 astrometry with an edge-on 

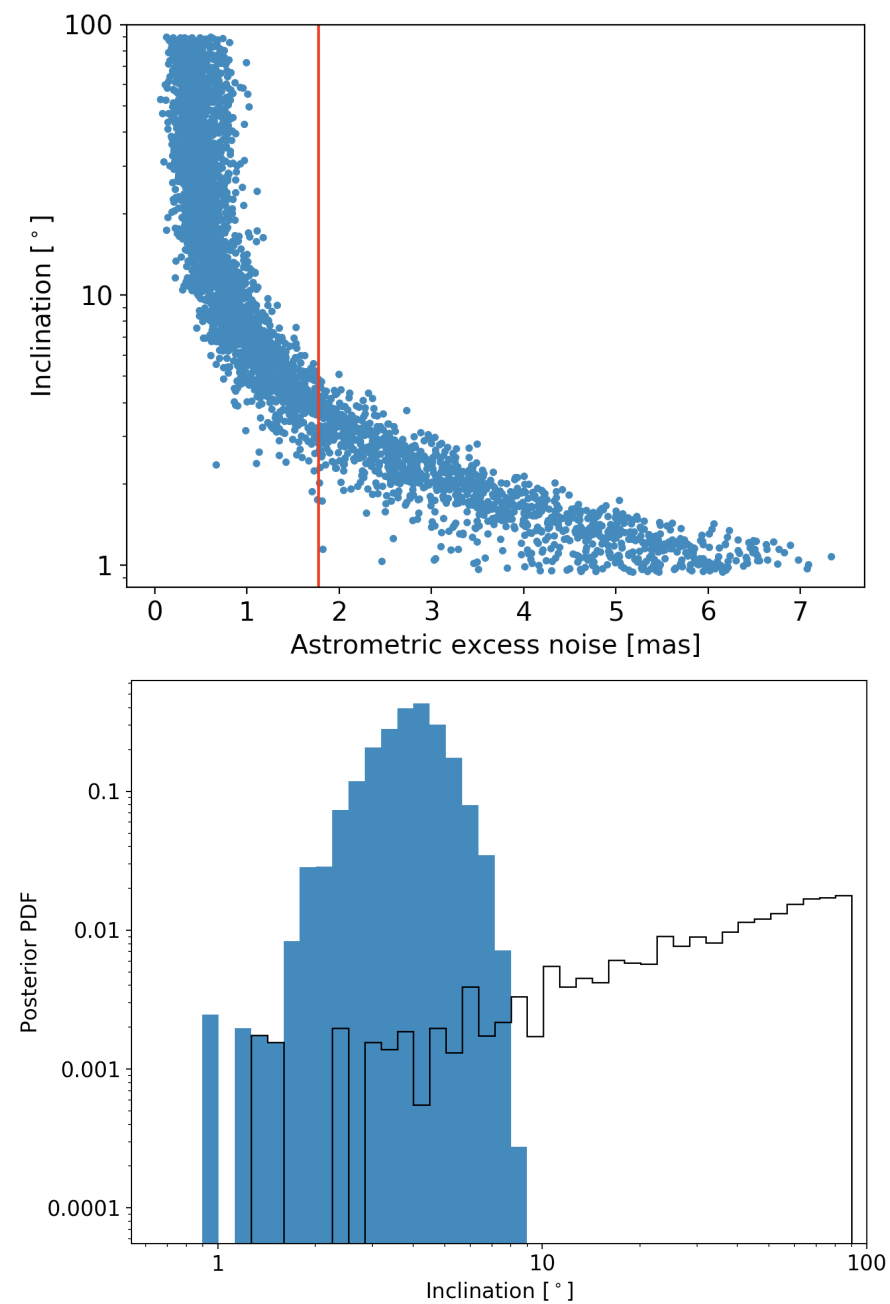

Fig. 7. Examples of situation \#1 with 30 Ari B b, primary dataset, $P=$ 335 days, $a_{\star} \sin i=0.17$ mas, and $\varepsilon_{\mathrm{DR} 1}=1.78$ mas. Top panel: $I_{\mathrm{c}}$ plotted against the derived $\varepsilon_{\text {simu }}$ for every simulations (blue points). The red line shows $\varepsilon_{\mathrm{DR} 1}$. Bottom panel: the $I_{\mathrm{c}}$ posterior PDF in blue, compared to the $\sin I_{\mathrm{c}}$ prior PDF (black solid line).

configuration, as revealed by the low 0.05 acceptance ratio of the MCMC run. The $1 \sigma$ bounds of the mass of HD $96127 \mathrm{~b}$ are thus questionable and its true nature is still uncertain.

The results for the single source from the secondary dataset of Gaia DR1 within situation \#1, HD $16760 \mathrm{~b}$, are given in Table 8, and illustrated in Fig. 9. HD 16760 b (Bouchy et al. 2009; Sato et al. 2009), the first companion with a possible planetary mass discovered with the SOPHIE spectrograph (Perruchot et al. 2011), is not a planet. With a parallax of 14 mas and an astrometric excess noise of 2.99 mas, we found its astrometry to be rather compatible with an $\mathrm{M}$ dwarf whose true mass is larger than $13.5 M_{\mathrm{J}}$ at $3 \sigma$.

\subsubsection{Situation \#2: Upper-limit constraint on companion mass}

The orbit inclination of 18 companions from 17 different systems in the detection sample cannot be fully determined using GASTON. For those orbits, the simulated astrometric excess noise is often compatible with $\varepsilon_{\mathrm{DR} 1}$ from $I_{\mathrm{c}}=90^{\circ}$ down to $\sim 0^{\circ}$. Accounting for the $\sin I_{\mathrm{c}}$ prior probability distribution on the inclination, the MCMC leads to a posterior distribution for which the $3 \sigma$ upper bound on inclination is located beyond $89.5^{\circ}$. More accurately, the posterior distribution on their orbit inclination and mass are mainly fixed by the $\sin I_{\mathrm{c}}$ prior on inclination.

As presented in Table 8, all these candidates are possible planets at the $1 \sigma$ limit. Excluding the transiting planets that are known to be bona fide planets on edge-on orbits, only two of these candidates have a true mass below, but close to, the deuterium burning limit of $13.5 M_{\mathrm{J}}$. These candidates are HD 164595 b (Courcol et al. 2015) and HD 185269 b (Moutou et al. 2006; Johnson et al. 2006), which have masses smaller than $12.9 M_{\mathrm{J}}$ and $12.6 M_{\mathrm{J}}$ at $3 \sigma$, respectively. Those two thus seem likely to be actual planets with masses in the Neptunian $\left(0.06 M_{\mathrm{J}}\right.$ for HD $164595 \mathrm{~b})$ and Jupiterian $\left(1.12 M_{\mathrm{J}}\right.$ for HD $\left.185269 \mathrm{~b}\right)$ domains.

We note however that HD 164595 is a duplicate source in the Gaia DR1. Its astrometric excess noise, and thus the mass of HD 164595 b, might be underestimated (see the discussion on this specific issue in Sect. 3.1). Moreover, for both companions, the simulated astrometric excess noise is compatible with $\varepsilon_{\mathrm{DR} 1}$ on a large range of inclinations (Figs. 10 and 11). The posterior distributions of $I_{\mathrm{c}}$ and $M_{\mathrm{c}}$ are essentially due to the prior distribution on $I_{\mathrm{c}}$. If the actual prior distribution of $I_{\mathrm{c}}$ is biased toward $0^{\circ}$ (see the related discussion in Sect. 6.2), it cannot be excluded that the masses of HD $164595 \mathrm{~b}$ and HD $185269 \mathrm{~b}$ are larger than $13.5 M_{\mathrm{J}}$.

Seven companions are transiting planets. These are HAT-P21 b (Bakos et al. 2011), WASP-11 b (West et al. 2009; Bakos et al. 2009), WASP-17 b (Anderson et al. 2010; Triaud et al. 2010), WASP-131 b (Hellier et al. 2017), WASP-157 b (Močnik et al. 2016), and K2-34 b (Hirano et al. 2016; Brahm et al. 2016) in the primary dataset, and K2-110 b (Osborn et al. 2017) in the secondary dataset. Gaia DR1 measurements all are compatible with the edge-on configurations. The MCMC acceptance rates are smaller than 0.01 with a star semimajor axis smaller than $1 \mu$ as. It can be excluded that Gaia will truly detect the reflex motion of these stars due to their transiting exoplanets.

Two exoplanet candidates are part of a common multiple system, HD 154857 b \& c (McCarthy et al. 2004; Wittenmyer et al. 2014). The Gaia observations are compatible with an edge-on inclination and masses of 2.2 and $2.5 M_{\mathrm{J}}$. At $1 \sigma$ the posterior distributions, which conform to the $\sin I_{\mathrm{c}}$ prior distribution on $I_{\mathrm{c}}$, allow inclinations as low as $20^{\circ}$ with masses as large as $6 M_{\mathrm{J}}$, but at $3 \sigma$ their mass could be as large as 135 and $175 M_{\mathrm{J}}$. Both companions are thus possible Jupiter-mass planets with masses within $2-6 M_{\mathrm{J}}$, but their true nature could not be confirmed.

\subsubsection{Situation \#3: Incompatible RV orbit and Gaia astrometry}

The GASTON results for the two companions within this situation are presented in Table 7. They are WASP-43 b (Hellier et al. 2011) and WASP-156 b (Demangeon et al. 2018), both transiting planets on compact orbit ( $P=0.8$ days and 3.8 days). In these two systems, none of the published companions are adequate for explaining observations by Gaia. The maximum astrometric excess noise that could be simulated from RV orbital parameters were 1.25 and 1.32 mas, respectively, well below the $\varepsilon_{\mathrm{DR} 1}$ of these two sources, which are 2 mas and 1.5 mas, respectively. These two sources from the secondary dataset are not mentioned as duplicated sources in the DR1 database.

There are three possible scenarios for explaining this RVGaia discrepancy. First, the value of the astrometric excess noise could depend on the presence of fortuitous outliers. With a number of astrometric measurements $\sim 50$ per source, outliers of 


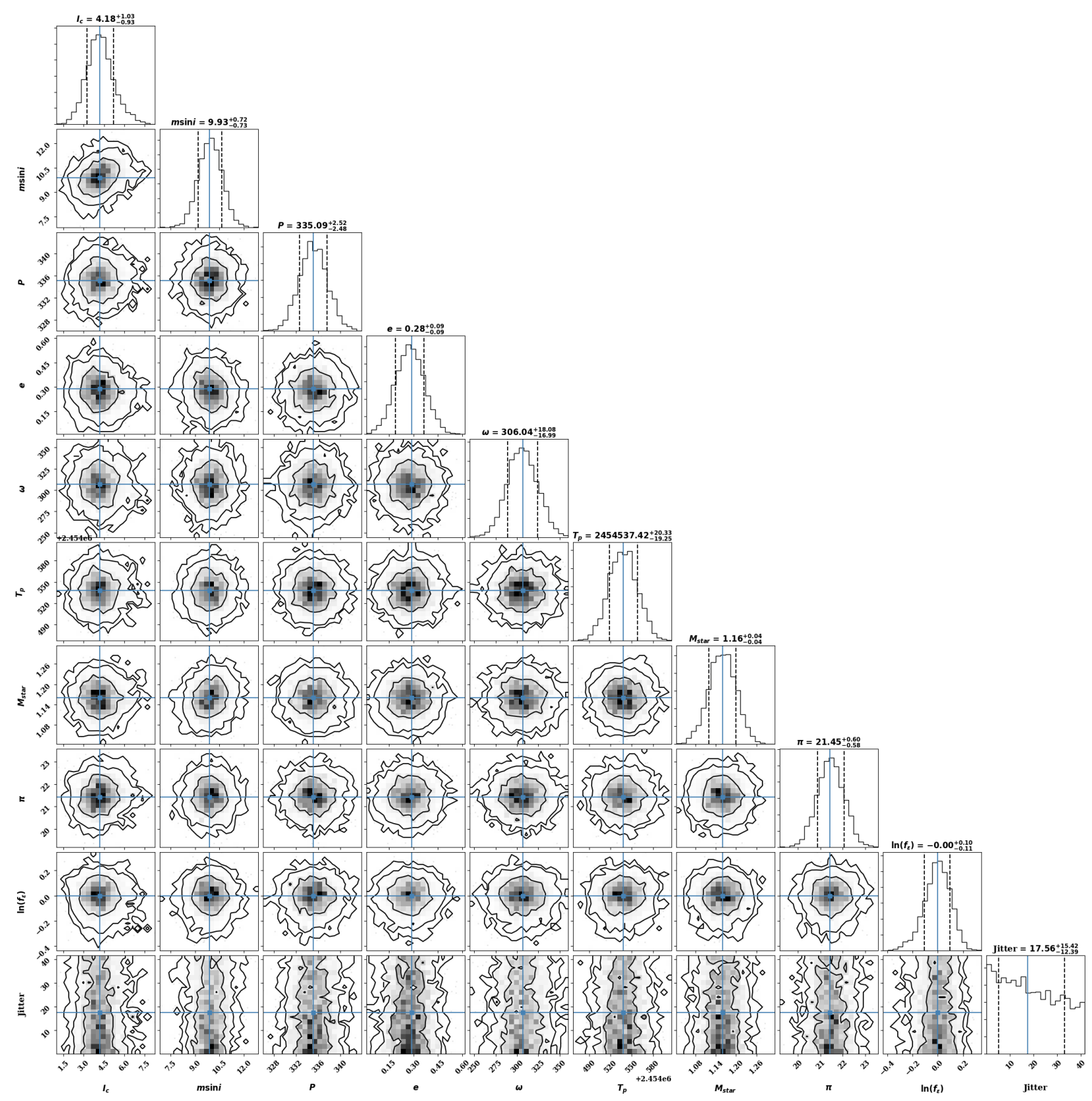

Fig. 8. Corner plot of posterior distributions for all explored parameters with the MCMC for 30 Ari B b from situation \#1 (Sect. 5.2.1).

several mas could slightly inflate $\varepsilon_{\mathrm{DR} 1}$ with a discrepancy of a few 0.1 mas. Outliers larger than $4.8 \varepsilon_{\mathrm{DR} 1} \sim 10$ mas (see note 7 in Lindegren et al. 2016) are flagged as "bad" during the DR1 reduction and discarded. Therefore, the discrepancy observed in Table 7 for the two companions between the highest $\varepsilon_{\text {simu }}$ and $\varepsilon_{\mathrm{DR} 1}$ of $0.2-0.7$ mas could be explained by numerous or large outliers. We cannot exclude this possibility without analyzing the time series, which will not be available until the final Gaia release in a few years.

Second, instrumental and modeling noises larger than those adopted in Sect. 3.4 could facilitate reaching the astrometric excess noise. For the astrometric data of secondary dataset targets the parallax and proper motion fit are not of good quality and could individually reach high astrometric excess noise, as indicated by the 90 th percentile $\varepsilon_{\mathrm{DR} 1}=2.3$ mas measured by Lindegren et al. (2016) in the full secondary dataset. Although plausible, as already discussed in Sect. 3.4, the good match between the distribution of simulated and observed $\varepsilon_{\mathrm{DR} 1}$ implies that the instrumental and modeling noise cannot be much larger than the adopted range of 0.4-0.9 mas in the present sample.

Third, a hidden outer companion to the system, unseen in the RV variations, could be responsible for the astrometric signal. This issue is discussed in Sect. 6.4. Although the presence of outliers cannot be excluded, this RV-Gaia discrepancy motivates the search for supplementary, yet hidden, companions in these systems.

\subsection{Non-detection sample: twenty-seven confirmed planets}

For a given RV orbit with given $m \sin i$ of the companion, an increasing true mass and thus decreasing orbital inclination imply increasing astrometric motion of the star. The nondetection of an astrometric excess noise larger than the defined threshold thus us to allow derive an upper limit on the true mass 

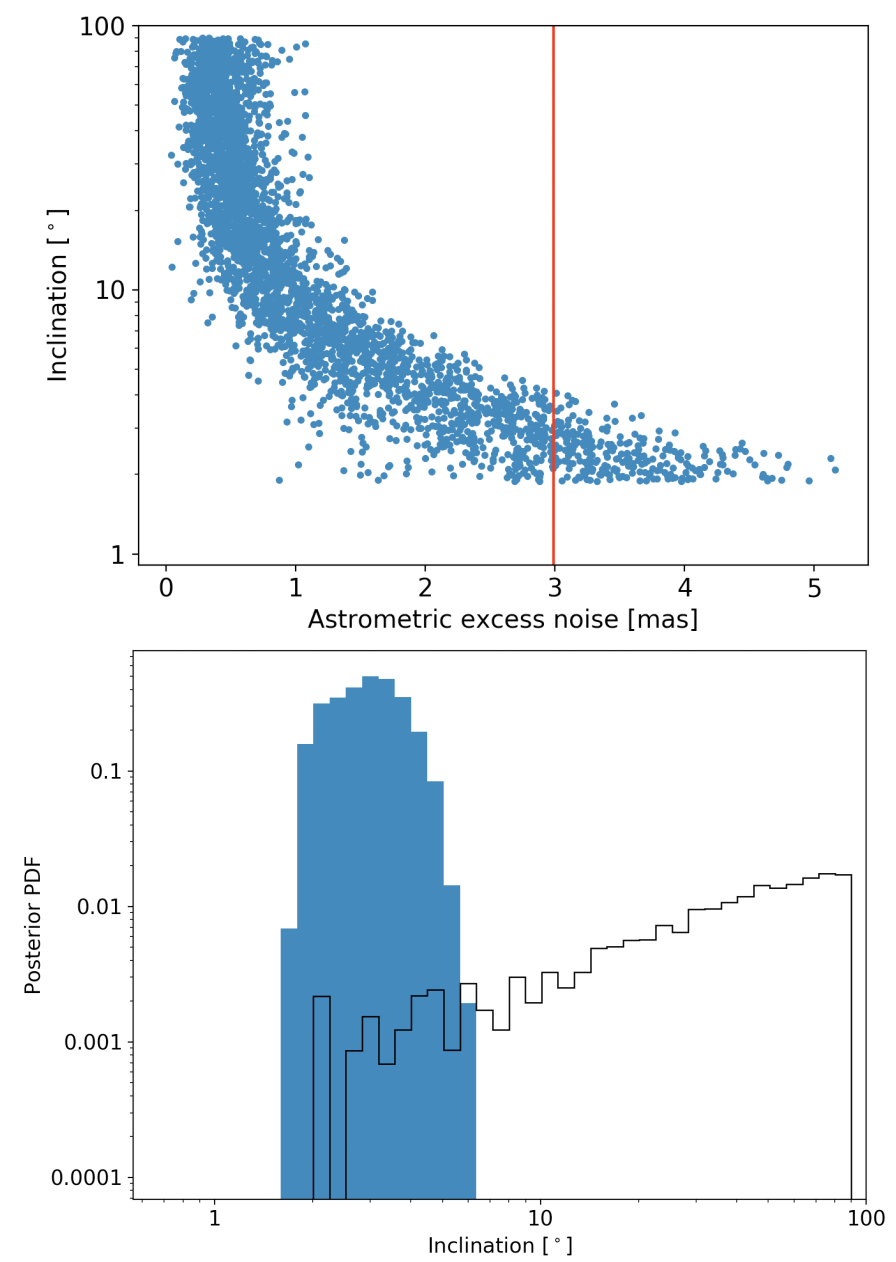

Fig. 9. Same as Fig. 7, illustrating situation \#1, with the secondary dataset source companion HD $16760 \mathrm{~b}\left(P=465\right.$ days, $a_{\star} \sin i=$ 0.25 mas, and $\varepsilon_{\mathrm{DR} 1}=3$ mas).

of the companion and a lower limit on its orbital inclination with GASTON.

Among the 227 non-transiting companions of the nondetection sample, we constrained true masses lower than $13.5 M_{\mathrm{J}}$ within $3 \sigma$ confidence interval for a total of 27 companions. These are summarized in Table 9. Nine planets have a true mass lower than $5 M_{\mathrm{J}}$ and 19 have a true mass lower than $10 M_{\mathrm{J}}$.

We confirm that six multiple systems contain several true planets. These are HD 10180, HD 176986, HD 181433, HD 215152, HD 7924, and HD 40307. In the six-planet systems, HD 10180, we can confirm that the a priori less massive companions c $\left(P=5.8\right.$ days, $\left.m \sin i=0.041 M_{\mathrm{J}}\right), \mathrm{d}(P=16.4$ days, $\left.m \sin i=0.037 M_{\mathrm{J}}\right)$, and $\mathrm{g}\left(P=602\right.$ days, $\left.m \sin i=0.067 M_{\mathrm{J}}\right)$ are planets with a mass that is strictly lower than $12 M_{\mathrm{J}}$ at $3 \sigma$. Figure 12 shows the $\varepsilon_{\text {simu }}-I_{\mathrm{c}}$ relationship and $I_{\mathrm{c}}$ posterior distribution for HD $10180 \mathrm{c}$. A study of the effect of mutual inclinations on the stability of this system led Lovis \& Fischer (2010) to constrain the masses of the planets within a factor of 3, with $I_{\mathrm{c}}>10^{\circ}$ for all planets. While our result is not as restrictive, it excludes a full face-on inclination with $I_{\mathrm{c}}>0.2^{\circ}$ at $3 \sigma$ and confirms planetary mass for planets $\mathrm{c}, \mathrm{d}$, and $\mathrm{g}$.

Among the 200 other candidate planets, as summarized in full Table 9 (available at the CDS), 103 companions can be confirmed substellar but may be as massive as BDs with a mass strictly smaller than $85 M_{\mathrm{J}}$ at $3 \sigma$, and 59 others have a mass upper limit within the M-dwarf domain. For the remaining 48
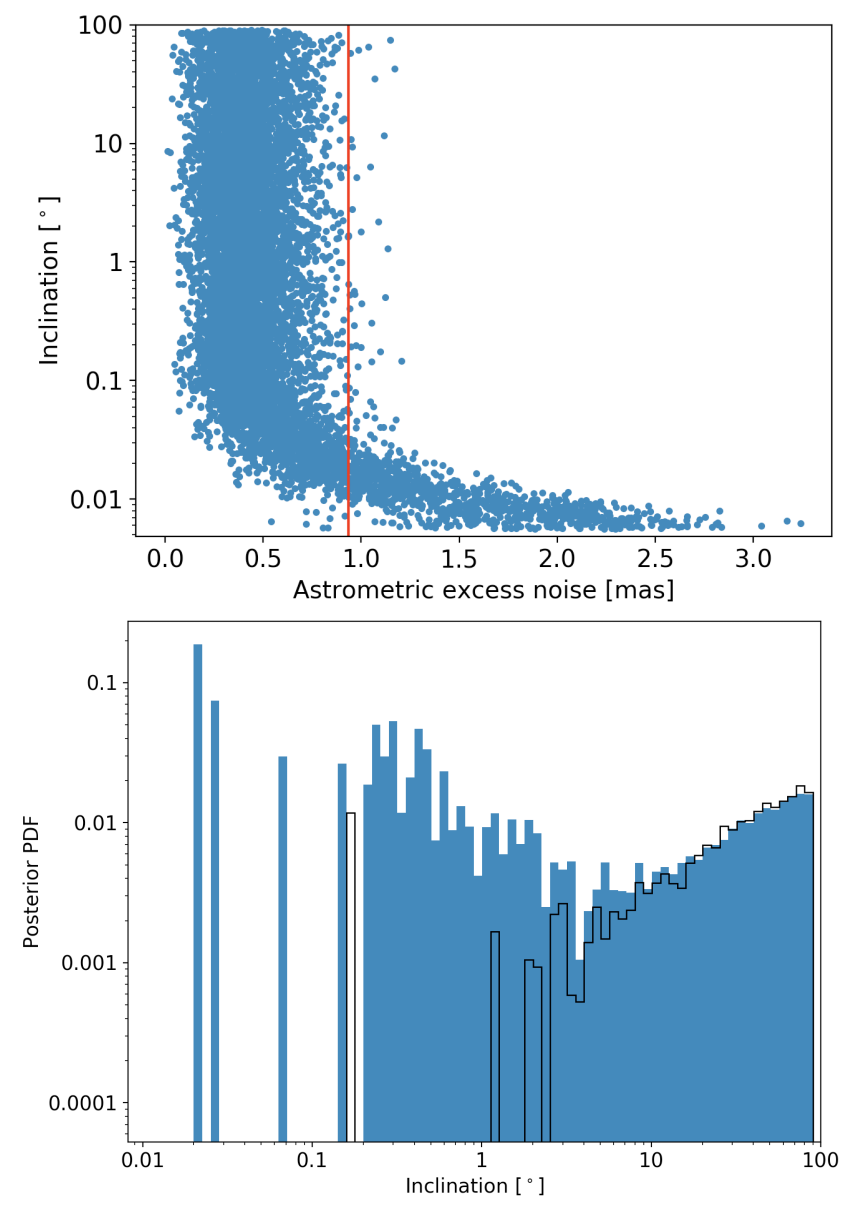

Fig. 10. Illustration of situation $\# 2$ with HD $164595 \mathrm{~b}, P=40$ days, $a_{\star} \sin i=0.0004$ mas, and $\varepsilon_{\mathrm{DR} 1}=0.93$ mas. Many simulations from $I_{\mathrm{c}}=0.1^{\circ}$ up to $90^{\circ}$ are compatible with $\varepsilon_{\mathrm{DR} 1}$. This reflects in the comparison of the $I_{\mathrm{c}}$ posterior to the $\sin I_{\mathrm{c}}$ prior PDFs shown in black.

companions, GASTON could not converge within the 50000 steps, with an autocorrelation length larger than 1000. At the end of the GASTON run, the posterior distributions for all of these companions led to an upper limit on the mass larger than $13.5 \mathrm{M}_{\mathrm{J}}$. This non-convergence is due to a large astrometric excess noise that is, however, smaller than the detection limit. Simulations are less often compatible with $\varepsilon_{\mathrm{DR} 1}$, GASTON thus needs more time to converge. Their nature is undetermined between planet, BD, and $\mathrm{M}$ dwarf. We are not publishing the obtained results for those 48 candidates.

While most of companions with a mass possibly greater than $13.5 M_{\mathrm{J}}$ have large orbital periods, 30 of these have an orbital period smaller than 100 days. Those are possible BD located within the driest region of the BDs detection desert (Kiefer et al. 2019). They are particularly interesting objects that need to be further characterized to better constrain the shores of the BD mass-period phase space.

\section{Discussion}

\subsection{Revised mass for nine companions}

Figure 13 summarizes the corrected mass derived with GASTON compared to the initial $m \sin i$ as given in the exoplanets.org database. The firm measurements for the nine companions identified in Sect. 5.2.1 lead to true masses significantly 

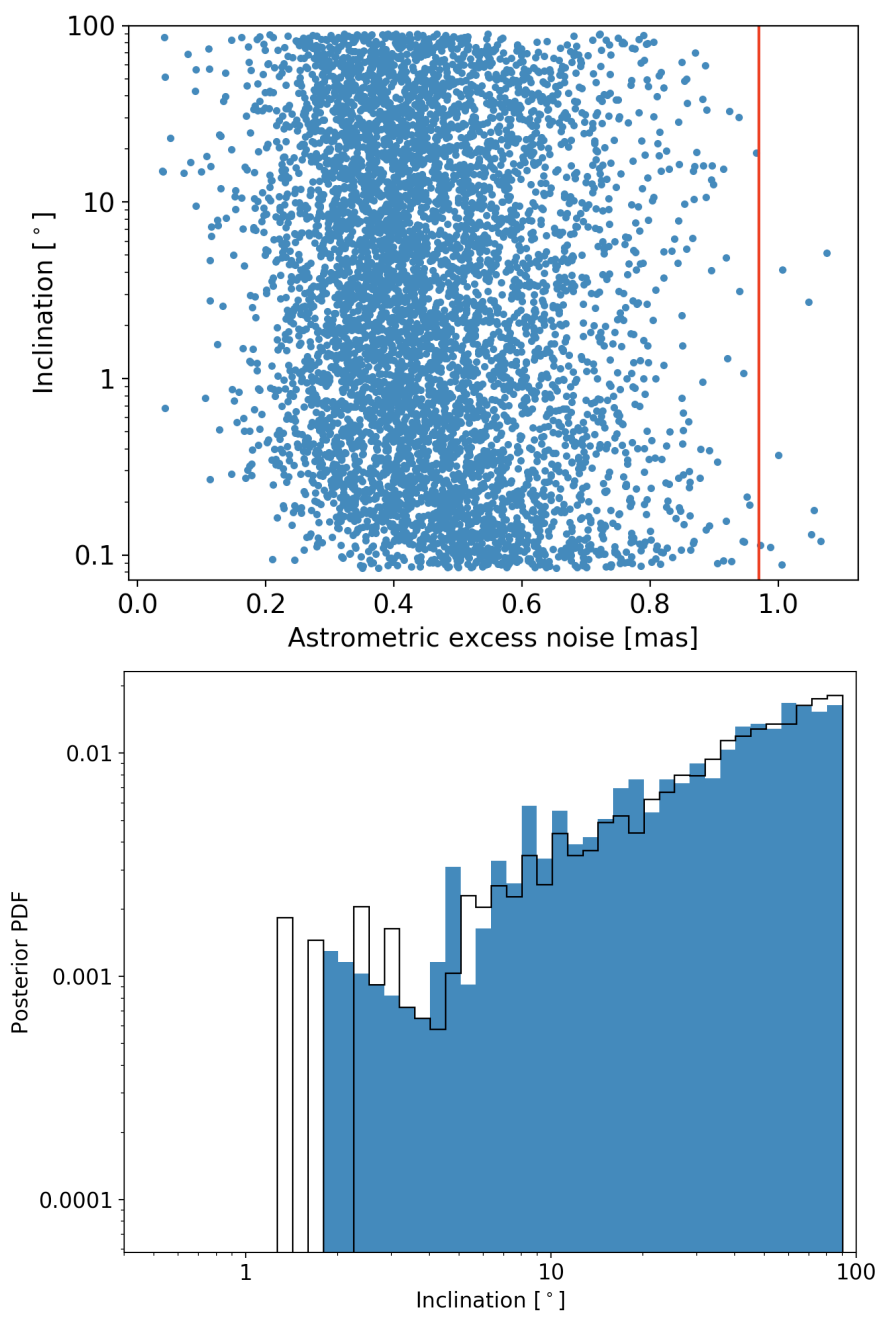

Fig. 11. Same plot as Fig. 10, illustrating situation \#2, with HD $185269 \mathrm{~b}$ $\left(P=6.8\right.$ days, $a_{\star} \sin i=0.001 \mathrm{mas}$, and $\left.\varepsilon_{\mathrm{DR} 1}=0.97 \mathrm{mas}\right)$. Many simulations from about $I_{\mathrm{c}}=0.1^{\circ}$ up to $90^{\circ}$ are compatible with $\varepsilon_{\mathrm{DR} 1}$ owing to noise. Smaller inclinations are rejected because the mass and luminosity are too large for the companion. The posterior distribution in the bottom panel is fully compatible with the prior sin $I_{\mathrm{c}}$ PDF.

different from the $m \sin i$ with an non edge-on inclination. Their revised masses are generally between $10 M_{\mathrm{J}}$ and $500 M_{\mathrm{J}}$, as are the $3 \sigma$ upper limits reported for companions from situation \#2 and in the non-detection sample.

This shows that Gaia will be best at detecting astrometric motions due to companions beyond $\sim 10 M_{\mathrm{J}}$. But with improved precision in the future releases and the use of time series, it will certainly allow for the detection of Jupiter mass planets.

\subsection{Small inclinations $<4^{\circ}$}

To our knowledge, no exoplanet RV candidate from the exoplanets.org database was yet found with an inclination strictly lower than $4^{\circ}$. The exoplanet with the smallest known orbital inclination is Kepler-419c with $I_{\mathrm{c}}=2.5 \pm 3^{\circ}$, thanks to transit timing variations (Dawson et al. 2014). In Table 8, among the nine non-transiting systems with a firmly detected inclination, and accounting for their $3 \sigma$ bounds, we find zero companions with $I_{\mathrm{c}}$ strictly smaller than $1^{\circ}$; one companion, HD $148427 \mathrm{~b}$, with $I_{\mathrm{c}}$ strictly smaller than $2^{\circ}$; and four others with $I_{\mathrm{c}}$ strictly smaller than $4^{\circ}$. Many other companions from the detection and non-detection samples could have such
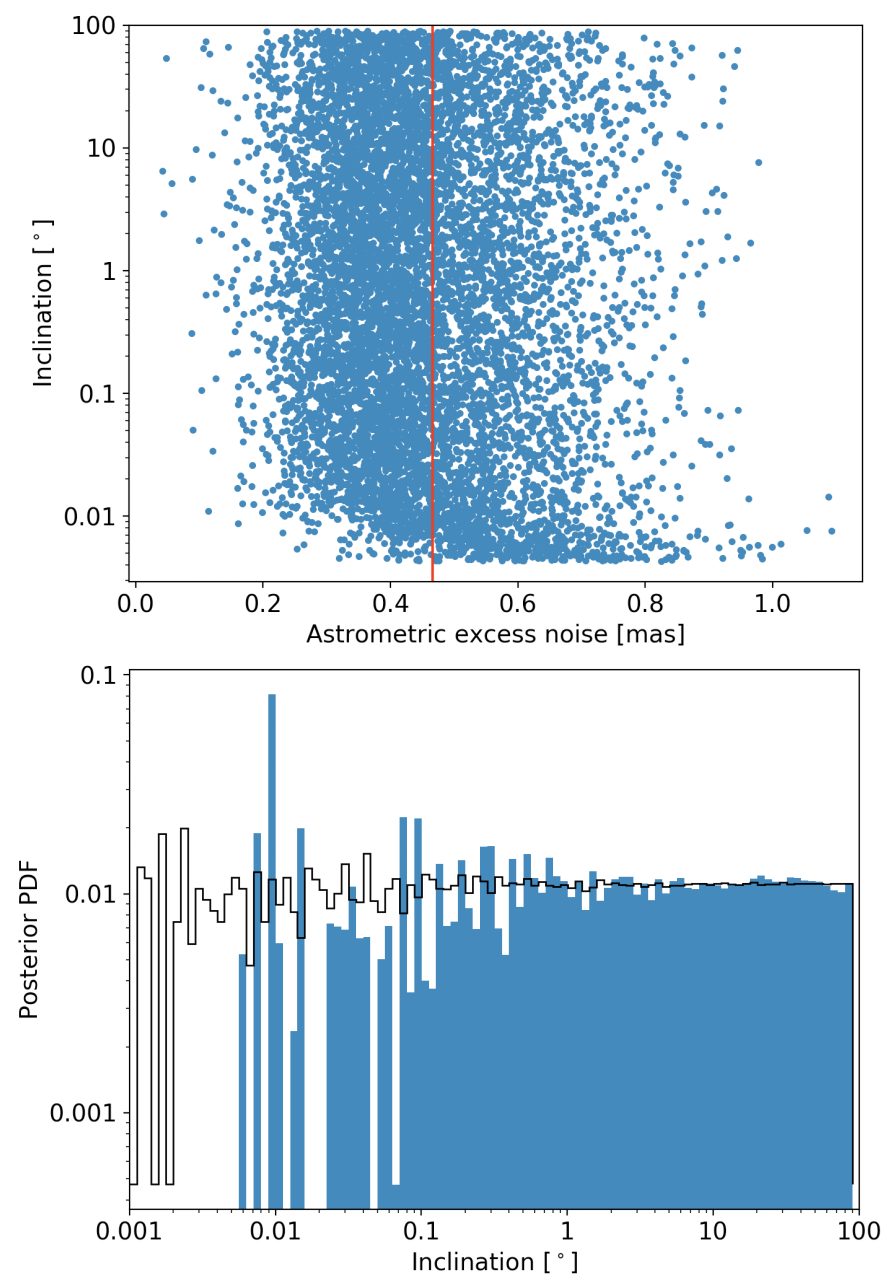

Fig. 12. Example of a confirmed planet from the non-detection sample with HD $10180 \mathrm{c}$ ( $P=5.8$ days, $m \sin i=0.041 M_{\mathrm{J}}, \varepsilon_{\mathrm{DR} 1}=0.47$ mas $)$. Top and bottom panels: same as Fig. 7, only the black line in the bottom panel now represents inclinations drawn from a uniform distribution.

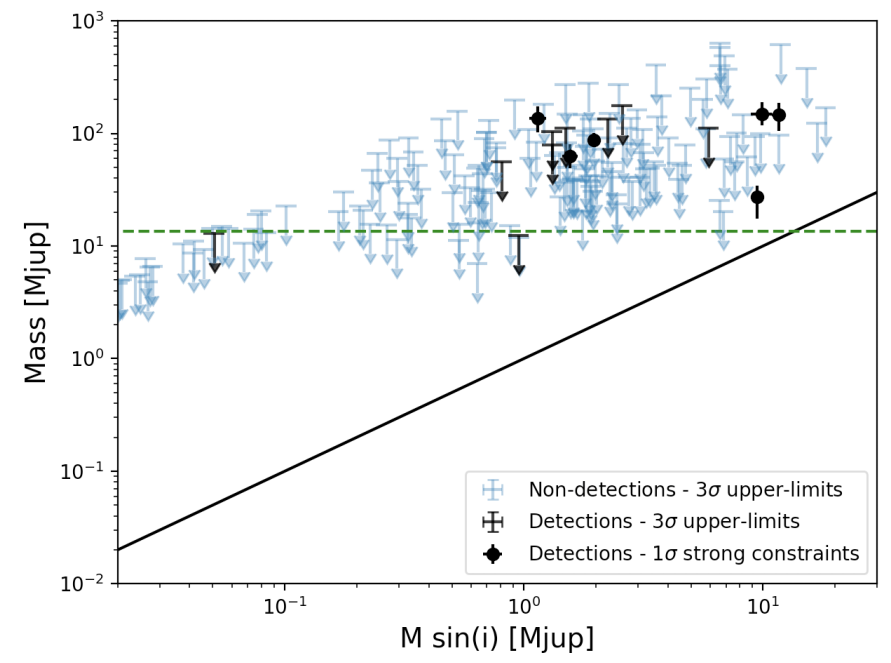

Fig. 13. Masses and upper limits derived with GASTON for 191 companions directly compared to the $m \sin i$ derived from RVs. The black solid line represents the relation $M_{\text {true }}=m \sin i$ obtained for an edge-on inclination of the orbits. The green dashed line indicates the $13.5-M_{\mathrm{J}}$ deuterium-burning limit. 
small inclinations, but could also be possibly larger than 1, 2, or $4^{\circ}$. Assuming isotropy of orbits within the $\sim 600$ known nontransiting RV exoplanets in the exoplanets.org database leads to less than 0.4 orbits with $I_{\mathrm{c}} \leq 2^{\circ}$ and less than 1.5 orbits with $I_{\mathrm{c}} \leq 4^{\circ}$. Finding more than one system with an orbit less inclined than $2^{\circ}$ and more than four with $I_{\mathrm{c}}<4^{\circ}$ suggests that the distribution of inclinations within exoplanet candidates deviates from a uniform distribution at least below $4^{\circ}$. This questions the isotropy of orbits within the population of discovered RV exoplanets advocated in, for example, Zucker \& Mazeh (2001b); Jorissen et al. (2001), and Tabachnik \& Tremaine (2002). It was indeed already noticed in Halbwachs et al. (2000) and Han et al. (2001) that RV-planet systems are possibly biased toward small $\sin i$.

The uniform distribution of inclinations certainly applies on a larger sample of systems than just RV exoplanets hosting systems. SB1 binary companions $\left(M_{\mathrm{c}}<0.6 M_{\star}\right)$ on orbits with inclinations $<2^{\circ}$ would most likely fall within the exoplanetary domain. A $\sin i<0.035$ would lead to $M \sin i<$ $20\left(M_{\star} / M_{\odot}\right) M_{\mathrm{J}}$. Several thousands of binary systems and stars were, and are still being, followed up for RV variations for many years. About 1300 SB1 binaries and about 600 RV systems, excluding all transiting planets that are biased to $90^{\circ}$, are collected in the SB9 database (Pourbaix et al. 2004). Probably twice as many are still being followed up, and have neither been characterized nor published yet. We thus estimate the full population of RV-monitored systems possibly, or actually, harboring planets or hidden binary component to reach at least 4000 individuals, among which FGK stars are the dominating class of stellar primaries. Assuming isotropy of orbits in this larger sample, we expect to observe at least $\sim 0.6$ systems with an inclination $\leq 1^{\circ}$, $\sim 2.4$ systems with $I_{\mathrm{c}} \leq 2^{\circ}$ and $\sim 9.7$ systems with $I_{\mathrm{c}} \leq 4^{\circ}$. This is in better agreement with our findings, validating the GASTON determination of orbital inclinations for RV companions with the Gaia DR1.

\subsection{Systems with edge-on transiting orbits}

In Sect. 3.3, we found 9 over 246 systems (i.e., 3.7\% of them) with transiting-only planets to have an astrometric excess noise larger than the defined thresholds for a significant astrometric motion. Six of these are systems around sources from the primary dataset. Given their edge-on orbit, with an expected astrometric semimajor axis of the photocenter on the order of a few $\mu$ as, it is technically impossible to detect the reflex motion of their host star with Gaia. But the measurement of $\varepsilon_{\mathrm{DR} 1}$ beyond the threshold suggests on the contrary that a significant astrometric motion was actually detected.

Running GASTON brings a solution to this inconsistency. For seven among nine companions, an edge-on astrometric orbit is compatible with the value of $\varepsilon_{\mathrm{DR} 1}$ because instrumental and measurement noises can pile up to produce an astrometric excess noise above the threshold. However, this compatibility is far from frequent in the MCMC runs, as shown by the low acceptance ratio in both cases $(<1.5 \%$; see Table 8$)$. Could it be that $\varepsilon_{\mathrm{DR} 1}$ is not due to instrumental and measurement noise for some of these candidates and leads to truly incompatible astrometric motion?

To test this possibility, we simulated many Gaia observations of non-accelerating stars, with $N_{\mathrm{FoV}}$ and $N_{\mathrm{AL}}$ drawn from the sample of 133 systems with transiting-only planets from the primary dataset, and the 113 systems with transiting-only planets from the secondary dataset. In the primary dataset, this results in 6 over 133 astrometric excess noise values beyond 0.85 mas, produced only from noise, with a probability of 0.0014 (i.e., below $3 \sigma$ ). Producing 5 over 133 astrometric excess noise values beyond 0.85 mas has a probability of 0.0072 (i.e., above $3 \sigma$ ). In the secondary dataset, the probability to produce 2 or 3 over 113 astrometric excess noise values beyond 1.2 mas is smaller than 0.0001 , while producing 1 over 113 is significantly more likely with a probability of $1.2 \%$.

Thus, Gaia detected a signal that cannot be fully explained by the combination of the published orbit and noise for at least one system from the primary dataset; this system could be K234 , which has the lowest MCMC acceptance rate of 0.002 . This could be the sign of an unseen and unknown outer companion in the system of K2-34, or a measurable effect of outliers (see the discussion in Sect. 5.2.3 for WASP-43 b and WASP-156 b).

In the secondary dataset, no more than 1 system among 113 could be simulated with an astrometric excess noise as large as 1.2 mas. We recall consistently obtaining a marginal compatibility of GASTON simulations with $\varepsilon_{\mathrm{DR} 1}$ only for K2-110 b (Sect. 5.2.2), and no compatibility at all for two other planets, WASP-43 b and WASP-156b (Sect. 5.2.3). We conclude that noise is the likely explanation for the astrometric excess noise of K2-110 b. As already discussed in Sect. 5.2.3, the most reasonable explanations for the large inconsistent $\varepsilon_{\mathrm{DR} 1}$ of WASP-43 and WASP-156 are either unmodeled outliers, or the presence of an outer companion in both systems. We conclude that followups of K2-34, WASP-43, and WASP-156 should be conducted to search for outer companions in these edge-on systems.

\subsection{Outer companions}

In the cases with the lowest acceptance rates in Table 8, it could be that the astrometric signal is better explained by the influence of another outer companion to the system, especially if as for HD 4203, a long-period RV-drift is detected (Kane et al. 2014). With a minimum mass of at least $2 M_{\mathrm{J}}$, this outer companion with an orbital period of several thousands of days (tens of year) might also be at the origin of the astrometric signal.

As mentioned in Sect. 6.3, the astrometric excess noise of few compact edge-on systems (K2-34, WASP-43 and WASP156) is difficult, and even impossible, to produce with our simulations. Thus, while outliers could explain the discrepancy of the RV orbit and Gaia astrometry, a more simple explanation could be the presence of an outer companion. There are no clues of long RV drifts in either of these systems, but this does not invalidate the hypothesis of an outer companion because the orbital inclination can be adjusted to make the RV signal vanish.

More generally, for any system in situation \#1 leading to a mass measurement of an RV companion, the presence of an unknown outer companion cannot be totally excluded. Nevertheless, as far as we know about these systems, the least complex solution is preferred, that is, the known RV orbit with a realistic inclination is responsible for the Gaia measurement of the star motion. A fine-tuned mass, semimajor axis, and inclination of an unknown orbit of an unseen companion would be necessary to explain the astrometric excess noise, while using the existing known companion on a known orbit is only required to fit a single parameter, the inclination.

We have shown in Sect. 3.3 that the astrometric excess noise is not correlated to the presence of an RV drift, and thus to the presence of an outer companion on an undetermined orbit. Thus, large astrometric excess noise does not imply the presence of an outer companion and conversely the presence of an outer companion does not imply large astrometric excess noise. It was already shown for HD 114762 b (Kiefer 2019) that the binary 


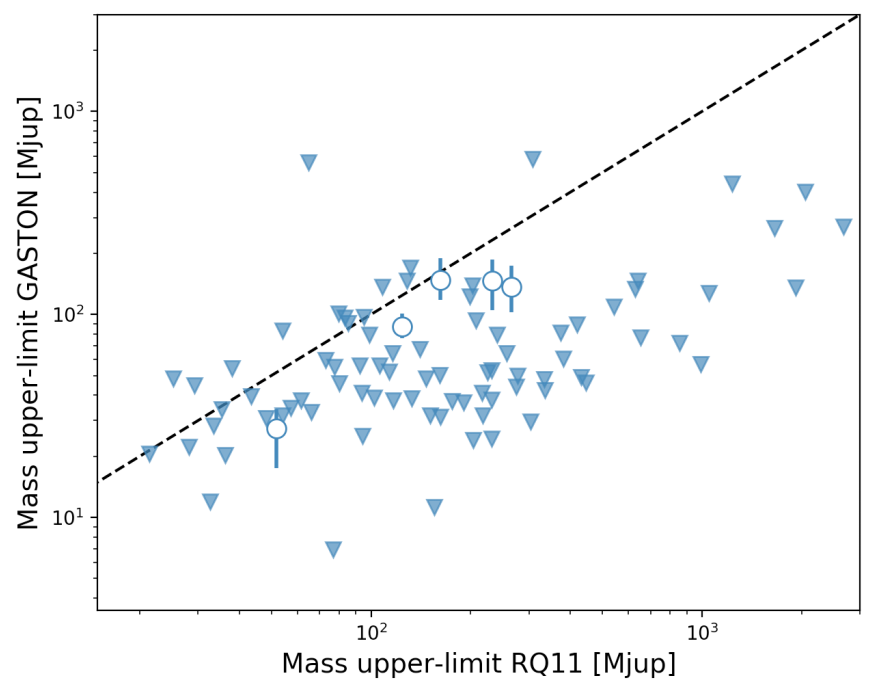

Fig. 14. Comparison plot of GASTON mass measurements (open blue circles) and other $3 \sigma$ upper limits (plain blue triangles) with Reffert \& Quirrenbach (2011), or RQ11, $3 \sigma$ mass upper limits for the same companions. The black dashed line represents the relation $M_{3 \sigma, \mathrm{GASTON}}=$ $M_{3 \sigma, \mathrm{RQ} 11}$.

companion HD 114762 B, a low-mass star at a separation of several hundred astronomical units and with an orbital period much larger than the Gaia DR1 campaign duration of 416 days, only has a minor impact on the motion of HD 114762 A. It was better explained by a small orbital inclination and larger mass of HD $114762 \mathrm{~A} b$.

Solving this issue is beyond the scope of the present paper, but learning from the specific case of HD 114762, we expect that outer companions, moreover not observed in the RV variations, with periods much greater than 416 days, could be neglected.

\subsection{Comparison with already published mass}

The true mass for 86 exoplanet candidates of our samples were also constrained in Reffert \& Quirrenbach (2011) using HipPARCOS-2 data. The results of the two studies are compared together in Fig. 14. This comparison shows that GASTON leads to better constraints with generally lower upper limits on the mass for 69 of the 86 companions. The Gaia DR1 astrometric excess noise is thus compatible the HIPPARCOS-2 astrometry even revealing smaller scatter and better astrometric precision. Among these 86 exoplanet candidates, Table 10 lists 5 companions with a well-constrained mass in the present study - 30 Ari B b, HD 114762 b, HD 141937 b, HD 148427 b, and HD 5388 b - for which the Reffert \& Quirrenbach (2011) analysis only led to upper limits on mass. The true mass and inclination that we obtain with GASTON all stand within the bounds they derived. We also list 5 companions for which GASTON could only derive limits - HD 190228 b, HD 87883 b, HD 142022 b, HD 181720 b, and HD 131664 b - but Reffert \& Quirrenbach (2011) published upper and lower bounds for both inclination and mass. We reduced the interval of possible mass for HD 142022 $\mathrm{b}$ and HD $181720 \mathrm{~b}$, now within 4.6-39 $M_{\mathrm{J}}$ and 6-32 $M_{\mathrm{J}}$, respectively, that is, in the giant planet or BD domain. We also confirm the upper bound on HD $87883 \mathrm{~b}$ mass, which ranges within 3 $21 M_{\mathrm{J}}$ at $3 \sigma$. HD $87883 \mathrm{~b}$ is thus most likely a giant planet on a long-period $7.5 \mathrm{yr}$ orbit.

Besides Reffert \& Quirrenbach (2011), we found several other publications of true mass and inclinations for 12 companions. These are summarized on Table 10 and discussed individually below.

HD5388b. The mass of this exoplanet candidate was already measured to be $62.2 \pm 19.9 M_{\mathrm{J}}$ with HIPPARCOS measurements (Sahlmann et al. 2011b). Our new mass estimation of $87.02_{-1080}^{+13.99} M_{\mathrm{J}}$ is $2 \sigma$ compatible with the estimation by Sahlmann et al. (2011b). This companion is thus indeed a likely massive BD.

HD 33636b. Bean et al. (2007) rejected the planetary nature of this candidate, with a mass determined in the M-dwarf domain, $M=140 \pm 11 M_{\mathrm{J}}$, with an orbital inclination of $\sim 4^{\circ}$. Interestingly, the small astrometric excess noise measured by Gaia $\varepsilon_{\mathrm{DR} 1}=0.53$ mas leads to a probability for the mass of this companion to be higher than $93.5 M_{\mathrm{J}}$ is $0.27 \%$. The mass measurements from Gaia and FGS astrometry are thus incompatible at $3 \sigma$. However, with an inclination of $4^{\circ}$, our simulations could produce $\varepsilon_{\text {DR1 }}$ smaller than 0.53 mas with a probability of $0.7 \%$. Thus, the Gaia DR1 astrometric excess noise is compatible with results by Bean et al. (2007) at $3 \sigma$. The disagreement between the parallax of this measured by FGS and HIPPARCOS $(\sim 35-$ 36 mas) with the parallax measured by Gaia ( 34) may also explain the small $\varepsilon_{\mathrm{DR} 1}$ if part of the orbital motion was wrongly fitted as parallax motion.

HD 92788 b. Han et al. (2001) proposed a true mass of $45 M_{\mathrm{J}}$ with an inclination of $6.3^{\circ}$ for this Jupiter-mass candidate $\left(m \sin i=3.6 M_{\mathrm{J}}\right)$ on an Earth-like orbit $(P=325$ days $)$. Simpson et al. (2010) later proposed a derivation of the orbit inclination based on the assumption of coplanarity of the stellar equator and the companion orbit and by measuring the rotation speed of the star compared to its $v \sin i$. This led to a lower mass of 9-28 $M_{\mathrm{J}}$. This method is however not fully reliable as coplanarity of the stellar equator and the companion orbit is never a robust assumption. Both results are compatible with the $3 \sigma$ limit that we derived in this work $\left(I_{\mathrm{c}}>3.9^{\circ}, M_{\mathrm{b}}<54 M_{\mathrm{J}}\right)$, where $\varepsilon_{\mathrm{DR} 1}=0.32$ mas. This confirms that this companion is most likely a BD, if it is not a massive planet.

$H D 102195 b$. This companion on a four-day orbit was determined as planetary by Guilluy et al. (2019) by extracting the emitted spectrum of its atmosphere. They determined a Jovian mass of $0.46 M_{\mathrm{J}}$ with an orbit inclination of 72 $85^{\circ}$. The GASTON tool cannot confirm the planetary nature of HD $102195 \mathrm{~b}$, with a $3 \sigma$ limit on its mass of $187 M_{\mathrm{J}}$ and a minimum inclination of $0.15^{\circ}$.

$H D 114762 b$. The true mass of this source was already published in Kiefer (2019). The results that we find for this companion are compatible with those reported in Kiefer (2019) when HD $114762 \mathrm{~B}$ is not taken into account, validating that the modifications brought to GASTON (see Sect. 4) kept the results of such well-behaved case unchanged. The tentative estimation of the mass of this companion obtained by Han et al. (2001) from few HIPPARCOS points led to an inclination of $4.3^{\circ}$ and a companion mass of $145 M_{\mathrm{J}}$, in full agreement with our result.

$H D 128311 b$ \& $c$. This system has two known companions $b$ and $c$, with periods of 454 and 924 days, respectively, in an almost 2:1 resonance, with minimum masses of $1.5 M_{\mathrm{J}}$ and $3.2 M_{\mathrm{J}}$. The outermost companion $c$ was shown to be most likely planetary by McArthur et al. (2014) using HST/FGS precise astrometry, with an orbit inclination of $55.95 \pm 14.55^{\circ}$ and a mass of $3.789_{-0.43}^{+0.92} M_{\mathrm{J}}$. There are no constraints on the inclination or true mass of companion $b$, but an assumed coplanarity with planet $c$ orbit would imply a planetary nature as well with a mass 
Table 10. Comparison of results in the present studies to $1 \sigma$ or $3 \sigma$ upper limits published in other articles.

\begin{tabular}{|c|c|c|c|c|c|c|}
\hline \multirow[t]{2}{*}{ Companion } & \multicolumn{2}{|c|}{ Reffert \& Quirrenbach (2011) } & \multicolumn{2}{|c|}{ Other publications } & \multicolumn{2}{|c|}{ Present study } \\
\hline & $\begin{array}{c}I_{\mathrm{c}} \\
\left(3-\sigma ;{ }^{\circ}\right)\end{array}$ & $\begin{array}{l}\text { True mass } \\
\left(3-\sigma ; M_{\mathrm{J}}\right)\end{array}$ & $\begin{array}{c}I_{\mathrm{c}} \\
\left(1-\sigma ;{ }^{\circ}\right)\end{array}$ & $\begin{array}{l}\text { True mass } \\
\left(1-\sigma ; M_{\mathrm{J}}\right)\end{array}$ & $\begin{array}{c}I_{\mathrm{c}} \\
\left(1 \text { or } 3 \sigma ;{ }^{\circ}\right)\end{array}$ & $\begin{array}{c}\text { True mass } \\
\left(1 \text { or } 3 \sigma ; M_{\mathrm{J}}\right)\end{array}$ \\
\hline \multicolumn{7}{|c|}{ Well-constrained mass with GASTON at $1-\sigma$} \\
\hline 30 Ari B b & $(3.8,174.8)$ & $<162.2$ & & & $4.2_{-0.9}^{+1.0}$ & $147_{-29}^{+41}$ \\
\hline HD 114762 b & $(4.1,176.7)$ & $<233.0$ & $4.87_{-0.91}^{+1.11(a)}$ & $141_{-28}^{+35(a)}$ & $4.9_{-0.9}^{+1.8}$ & $147_{-42}^{+39}$ \\
\hline HD 141937 b & $(11.0,166.2)$ & $<51.7$ & & & $20.5_{-4.2}^{+12.5}$ & $27.4_{-9.9}^{+6.8}$ \\
\hline HD 148427 b & $(4.8,176.6)^{(\dagger)}$ & $<265$ & & & $0.51_{-0.11}^{+0.16}$ & $137_{-34}^{+37}$ \\
\hline HD 5388 b & $(1.0,179.0)$ & $<124.3$ & $178.3_{-0.7}^{+0.4(b)}$ & $62.2 \pm 19.9^{(b)}$ & $1.4 \pm 0.2$ & $87_{-11}^{+14}$ \\
\hline \multicolumn{7}{|c|}{$3 \sigma$ limits with GASTON } \\
\hline HD $33636 b$ & $(2.9,176.8)$ & $<207.3$ & $4.0 \pm 0.1^{(c)}$ & (c) $142 \pm 11$ & $>6.02$ & $<93.5$ \\
\hline HD $87883 b$ & $(4.9,34.9)$ & $(3.1,21.4)$ & & & $>4.9$ & $<21$ \\
\hline HD 92788 b & $(2.5,178.0)$ & $<113.6$ & $6.3 ;^{(d)}(0,22)^{(e)}$ & $45 ;^{(d)}(9,28)^{(e)}$ & $>4.0$ & $<52$ \\
\hline HD $102195 b$ & $(0.0,180.0)$ & - & $(72.5,84.79)^{(f)}$ & $0.46 \pm 0.03^{(f)}$ & $>0.21$ & $<133$ \\
\hline HD $128311 \mathrm{c}$ & $(7.5,171.1)$ & $<25.2$ & $55.950 \pm 14.553^{(g)}$ & $3.789_{-0.432}^{+0.924(g)}$ & $>4.0$ & $<49$ \\
\hline HD 130322 b & $(0.0,180.0)$ & - & $76_{-42}^{+14(e)}$ & $1.1_{-0.1}^{+1.0(e)}$ & $>0.59$ & $<108$ \\
\hline HD 131664 b & $(153.9,171.5)$ & $(42.3,131.6)$ & $55 \pm 33^{(h)}$ & $23_{-5}^{+26(h)}$ & $>6.6$ & $<170$ \\
\hline HD 136118 b & $(138,172.7)$ & $<95.3$ & $163.1 \pm 3.0^{(i)}$ & $42_{-18}^{+11(i)}$ & $>7.25$ & $<97$ \\
\hline HD 142022 b & $(4.2,49.1)$ & $(4.6,102.2)$ & & & $>4.348$ & $<38.84$ \\
\hline HD 154345 b & $(1.7,178.1)$ & $<32.6$ & $50_{-26}^{+40(e)}$ & $1.2_{-0.4}^{+1.3(e)}$ & $>4.7$ & $<12$ \\
\hline HD $177830 \mathrm{~b}$ & $(0.9,179.6)$ & $<225.2$ & $1.3^{(d)}$ & $55^{(d)}$ & $>1$ & $<79$ \\
\hline HD $181720 \mathrm{~b}$ & $(0.1,3.4)$ & $(6.1,217.9)$ & & & $>0.68$ & $<32$ \\
\hline HD 190228 b & $(2.5,40.8)$ & $(9.1,142.9)$ & $4.3_{-1.0}^{+1.8(j)}$ & $49^{(j)} \pm 15$ & $>1.56$ & $<111.4$ \\
\hline
\end{tabular}

Notes. Inclinations should be compared modulo $180^{\circ}$ since in the present study the prograde or retrograde orientation of the orbital motion cannot be determined. We present the $1 \sigma$ confidence interval for the inclinations and masses when they are well constrained in the present study and obtained with a $\sin I_{\mathrm{c}}$ prior distribution on the inclination, and the $3 \sigma$ limits obtained with a flat prior distribution otherwise. We believe there is an issue in Reffert \& Quirrenbach (2011) for this companion, since its $m \sin i$ is $\sim 1 M_{\mathrm{J}}$ and the inclination, and true mass $3 \sigma$ constraints are not compatible with each other. ${ }^{(a)}$ From Kiefer (2019); this values does not account for the astrometric motion of HD 114762 A due to the wide-binary component HD 114762 B. ${ }^{(b)}$ From Sahlmann et al. 2011b. ${ }^{\left({ }^{(}\right)}$From Bean et al. (2007) with astrometry from the HST/FGS. ${ }^{(d)}$ From Han et al. (2001). No confidence intervals are given. ${ }^{(e)}$ From Simpson et al. (2010). Derived from parent star rotation axis inclination, assuming coplanarity of stellar equator and planet orbit. ${ }^{(f)}$ From Guilluy et al. (2019) by extracting the spectra emitted by this non-transiting planet. ${ }^{(g)}$ From McArthur et al. (2014) with astrometry from the HST/FGS. ${ }^{(h)}$ From Sozzetti \& Desidera (2010) with HIPPARCOS astrometry. ${ }^{(i)}$ From Martioli et al. (2010) with astrometry from the HST/FGS. ${ }^{(j)}$ From Sahlmann et al. (2011a) with HIPPARCOS astrometry.

close to $2 M_{\mathrm{J}}$. Coplanarity is not generic, and it remains thus possible that planet $c$ is actually circumbinary, possibly leading to an interesting configuration in 2:1 resonance. The non-detection of the astrometric motion of the host star by the Gaia DR1 astrometric excess noise with $\varepsilon_{\mathrm{DR} 1}=0.6$ mas puts a $3 \sigma$ upper limit on the mass at $46 M_{\mathrm{J}}$ and $48 M_{\mathrm{J}}$ for companions $b$ and $c$. We can therefore exclude a stellar nature for planet $c$, in agreement with McArthur et al. (2014), as well as for object $b$, but this source could still be a BD.

$H D 130322 b$. As for HD $154345 \mathrm{~b}$, assuming the coplanarity of HD 130322's equator and companion $b$ orbit, Simpson et al. (2010) proposes a mass of $1.1 M_{\mathrm{J}}$ for this companion. The low astrometric excess noise of 0.3 mas for this source allows deriving with GASTON a 3- $\sigma$ upper-limit on the mass of HD $130322 \mathrm{~b}$ of $136 M_{\mathrm{J}}$. The planetary nature of this object cannot be confirmed here.

$H D 131664 b$. This 5 -yr period candidate BD $(m \sin i=$ $18 M_{\mathrm{J}}$ ) was characterized using HIPPARCOS astrometry by
Sozzetti \& Desidera (2010) and HIPPARCOS-2 data by Reffert \& Quirrenbach (2011). Both of these studies found a possible small orbital inclination for this companion down to $\sim 10-20^{\circ}$. Sozzetti \& Desidera (2010) could not reject an edge-on inclination, while Reffert \& Quirrenbach (2011) obtained inclinations smaller than $30^{\circ}$ at $3 \sigma$ with a mass of at least $42 M_{\mathrm{J}}$. The GASTON method cannot help in settling the true mass of HD $131664 \mathrm{~b}$, but constrains its mass to less than $170 M_{\mathrm{J}}$ at $3 \sigma$.

HD 136118b. Using the HST/FGS, Martioli et al. (2010) measured the astrometric motion of the F9V star HD 136118. They obtained an inclination of $163 \pm 3^{\circ}$ and a true mass for the exoplanet candidate of $42_{-18}^{11} M_{\mathrm{J}}$ instead of the $12 M_{\mathrm{J}}$ deduced from RV assuming an orbit seen edge-on. The $3 \sigma$ upper limit that we derived with GASTON for the true mass of HD $136118 \mathrm{~b}$ is close to the $3 \sigma$ upper limit derived from HIPPARCOS- 2 measurements by Reffert \& Quirrenbach (2011) about 95-97 $M_{\mathrm{J}}$. The Gaia DR1 astrometric excess noise of HD 136118 (0.51 mas) is thus compatible with the true astrometric motion $\sim 1.45$ mas of the host star due to companion "b". 
$H D 154345 b$. This long-period (3325 day) companion is a planet confirmed by Simpson et al. (2010) by measuring the rotation axis angle of the host star. But this conclusion relies on the hypothesis of coplanar orbital and stellar equator planes, which is never guaranteed. Our $3 \sigma$ limit based on $\varepsilon_{\mathrm{DR} 1}=0.35$ mas shows that HD $154345 \mathrm{~b}$ is a planet with a mass smaller than $11.6 M_{\mathrm{J}}$.

$H D 177830 \mathrm{~b}$. Its true mass was tentatively determined at $55 M_{\mathrm{J}}$ with an inclination of $1.3^{\circ}$ by Han et al. (2001) using HIPPARCOS data. This result is within the $3 \sigma$ limit that we derived $\left(I_{\mathrm{c}}>1.0^{\circ}, M_{\mathrm{b}}<79 M_{\mathrm{J}}\right)$, confirming that the $\varepsilon_{\mathrm{DR} 1}=$ 0.87 mas for this source incorporates a consequent fraction of real astrometric orbital motion.

HD 190228b. Using HIPPARCOS astrometry, this companion was previously identified as a BD with a mass of $67 \pm$ $29 M_{\text {J }}$ (Zucker \& Mazeh 2001a). Its mass was then reduced to $49 \pm 18 M_{\mathrm{J}}$ with an inclination of $4.3_{-1.0}^{\circ+1.8}$ and an orbit significance standing in the range $2-3 \sigma$ (Sahlmann et al. 2011a). Using GASTON and a sin $i$-prior on the inclination, we measured a $1 \sigma$ upper limit for the same mass of $<24 M_{\mathrm{J}}$ and a $3 \sigma$ upper limit of $111 M_{\mathrm{J}}$. The inclination is $>14^{\circ}$ at $1 \sigma$ and $>3.2^{\circ}$ at $3 \sigma$. Our result agrees with the most precise measurements of the mass of HD 190228 b, but cannot bring significant improvements. Given the astrometric orbit semimajor axis as large as 2 mas, Gaia certainly provides the best measurement for this $\mathrm{BD}$ once the astrometric series is available.

The global compatibility of the true masses derived with GASTON with the true masses already published for these systems validates the GASTON method and confirms this tool can lead us to better characterize candidate planetary systems.

\subsection{Updated mass-period diagram}

The masses derived with GASTON allow us to update the massperiod diagram of planet and BD companions. This diagram is represented in Fig. 15, compared to companions with true mass from the exoplanet.eu database and massive companions reported in Wilson et al. (2016) and Kiefer et al. (2019). We only selected systems within $60 \mathrm{pc}$ from the Sun, surrounding FGK host stars with masses within $0.52-1.7 M_{\odot}$, with a published inclination measurement. Such systems are objects of extensive surveys (e.g., Sahlmann et al. 2011a, Hébrard et al. 2016; Kiefer et al. 2019) with better observational completion and detection of planets and BD with mass larger than $1 M_{\mathrm{J}}$. We included any mass compatible with as much as $150 M_{\mathrm{J}}$ to encompass the surroundings of the substellar domain. We excluded GASTON masses of transiting planets, which are better determined in the Exoplanet. eu database. We also excluded the GASTON mass of candidates that host-star RVs have a longterm drift to remove possible bias due to an outer companion. Upper limits are not represented.

The mass measurements of the present study add new points to the mass-period $(M-P)$ diagram in the BD-to-stellar domain at orbital periods larger than 100 days. There still remains blank regions: the BD domain below 100-day period (Kiefer et al. 2019), the short-period Neptunian desert (Mazeh et al. 2016), and the observationally biased triangular area from short-period Earth-mass planets to long-period Jupiter mass planets.

In the BD domain, the $M-P$ distribution presents a strong cut in the region of $\mathrm{BD}$ companions at $\sim 100$ days. But below 100 days, several tens of other companions which mass cannot be well-constrained may reside in the BD mass regime. In Fig. 16, we included the $3 \sigma$ upper limits derived with GASTON around

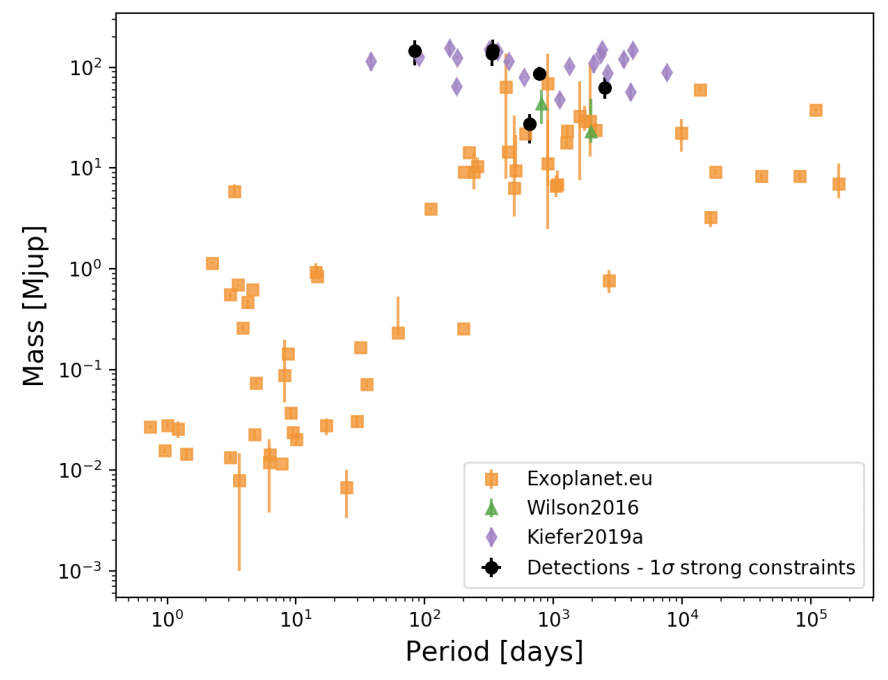

Fig. 15. Mass-period diagram from planets up to low-mass $M$ dwarfs $\left(\sim 150 M_{\mathrm{J}}\right)$. The true masses derived with GASTON for non-transiting planets and systems without RV drift are represented as black circles. In comparison, the true mass and period of companions in the exoplanet. eu database are plotted as orange squares, supplementary BD and low-mass M dwarfs published in Wilson et al. (2016) are plotted in green triangles, and Kiefer et al. 2019 companions are shown as purple diamonds. Only systems within $60 \mathrm{pc}$ from the Sun and surrounding FGK host stars $\left(0.52<M_{\star}<1.7 M_{\odot}\right)$ are represented.

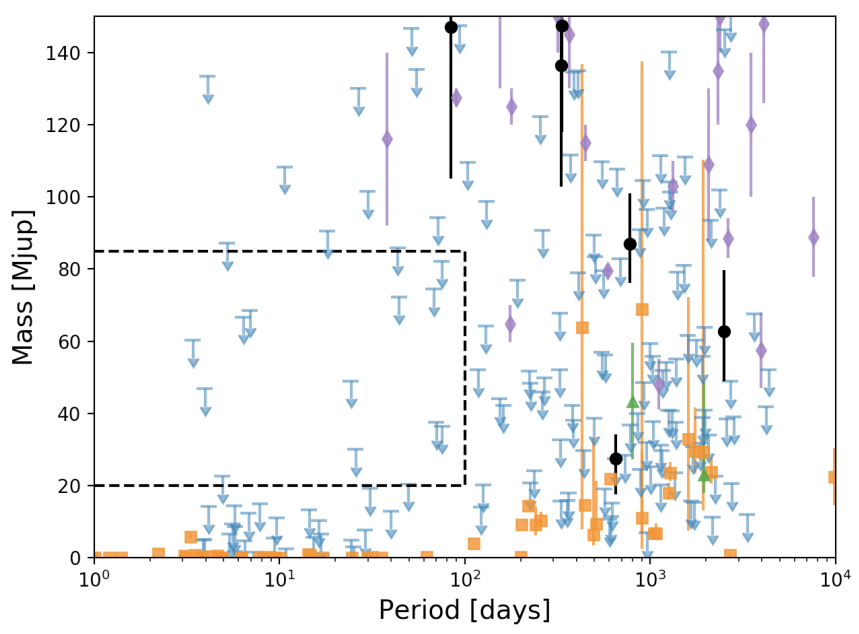

Fig. 16. Same diagram as Fig. 15, but focused on the $0-90 M_{\mathrm{J}}$ region with a linear scaling of the $y$-axis and centered on the BD domain. The $3 \sigma$ upper limits on mass derived by GASTON are shown as blue downward arrows.

and in the BD domain. Even then, the region bounded by masses 20-85 $M_{\text {J }}$ and periods 0-100 days remains significantly emptier than its surrounding. This tends to confirm the most recent estimation of the BDs desert boundaries (Ma \& Ge 2014; Ranc et al. 2015; Kiefer et al. 2019).

\subsection{Star-host metallicity and orbital eccentricity distributions in the $B D$ domain}

Brown dwarf companions stand at the boundary between stellar binaries and giant planets. It remains unknown if some BD companions belong to one population or another or if BDs have a main formation channel. 


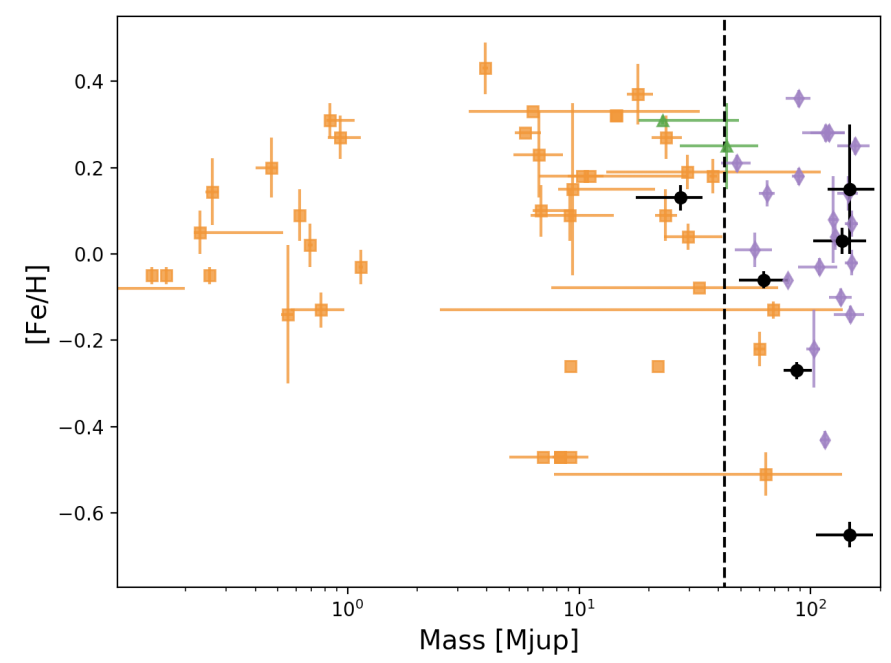

Fig. 17. Mass-metallicity diagram from planets up to low-mass $M$ dwarfs $\left(\sim 150 M_{\mathrm{J}}\right)$. The symbols are the same as in Fig. 15. The dashed line indicates the $42.5 M_{\mathrm{J}}$ mass limit derived by Ma \& Ge (2014).

Core-accretion scenarios (Pollack et al. 1996) predict that giant planets have more difficulty forming around metaldeficient stars (Ida \& Lin 2005) and strong observational evidences show that giant planets occur less frequently about stars with subsolar metallicity (Fischer \& Valenti 2005; Mayor et al. 2011). On the other hand, the formation of stellar binaries by gravitational instability does not depend on the metallicity of the host star and it is expected that binary companions have the same metallicity distribution whatever their mass (Maldonado \& Villaver 2017).

The study of eccentricity distributions in the BD domain by Ma \& Ge (2014) revealed the existence of a sharp transition at $\sim 42.5 M_{\mathrm{J}}$. Below $42.5 M_{\mathrm{J}}$ the eccentricity distribution is consistent with mass-limited eccentricity pumping by planet-planet scattering (Rasio \& Ford 1996), and beyond $42.5 M_{\mathrm{J}}$ the eccentricity distribution of BD companions is similar to that of binary stars (Halbwachs et al. 2003). A consistent transition at similar mass was found by Maldonado \& Villaver (2017) in the distribution of host-star metallicities. The host-star metallicity of BD with a mass $>42.5 M_{\mathrm{J}}$ spans a large range of values from subsolar to super-solar, while those with mass $<42.5 M_{\mathrm{J}}$ have host-star metallicities more similar to those of giant exoplanets with a prevalence for metal-rich hosts. This limiting mass of $\sim 42.5 M_{\mathrm{J}}$ could thus be separating low-mass BDs that are formed like planets by core accretion from high-mass BDs formed like stars by gravitational instability in molecular clouds.

In this work, we add the new GASTON measurements to exoplanet.eu companions, and the published companions in Wilson et al. (2016) and Kiefer et al. (2019) to obtain metallicity and eccentricity distributions with respect to true masses in Figs. 17 and 18. We select, as in Sect. 6.6, systems within $60 \mathrm{pc}$ from the Sun, surrounding FGK host stars with masses within $0.52-1.7 M_{\odot}$, and with a published inclination measurement. Metallicity, or $[\mathrm{Fe} / \mathrm{H}]$, measurements are taken from the exoplanets.org database for the sample studied in the present paper, from exoplanet.eu for the corresponding sample, and from the Wilson et al. (2016) and Kiefer et al. (2019) for the rest of the considered companions.

Beyond the BD domain, metallicity reaches subsolar values, while giant planets are indeed found preferably around stars with super-solar metallicity. No clear boundary can be derived

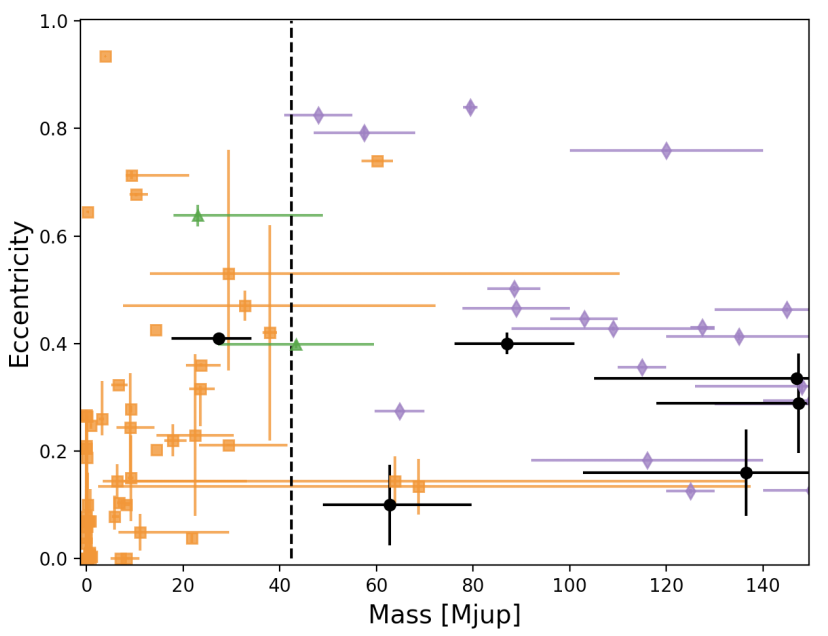

Fig. 18. Mass-eccentricity diagram from planets up to low-mass M dwarfs $\left(\sim 150 M_{\mathrm{J}}\right)$, with a linear scale as in Ma \& Ge (2014). The symbols are the same as in Fig. 17.

from the still sparse distribution of measured companion mass, although a transition could be occurring about $50 M_{\mathrm{J}}$ in agreement with the $42.5 M_{\mathrm{J}}$ found by Maldonado \& Villaver (2017). The distribution of eccentricity with companion mass in Fig. 18 does not exhibit a well-defined transition at $42.5 M_{\mathrm{J}}$, as reported in Ma \& Ge (2014). Nevertheless, four BDs with $M>45 M_{\mathrm{J}}$ stand above $e=0.7$, while all $\mathrm{BD}$ companions have $e<0.7$ below $45 M_{\mathrm{J}}$. The eccentricity distribution within our sample thus seems to match with that of Ma \& Ge (2014).

Our current sample of exoplanets, BDs, and low-mass Mdwarf companions around FGK stars at less than $60 \mathrm{pc}$ of the Sun with a true mass measured still needs to be populated. Nevertheless, our sample agrees with previous non-volume limited studies on a transition in the BD domain at a mass of $\sim 42.5 M_{\mathrm{J}}$. This critical mass possibly separates two populations of BDs, those formed like stars from those formed like planets, and mainly follows predictions of the core-accretion scenario.

\section{Conclusions}

We used the GASTON method developed in Kiefer et al. (2019) and Kiefer (2019) with Gaia DR1 data to determine the true mass of the $911 \mathrm{RV}$-detected exoplanet candidates published in the exoplanets.org database. Reliable DR1 data were found for the host stars of 755 companions. Among those, a total of 29 companions induce an orbital motion of their host star significant enough to be detected as large astrometric excess noise, constituting a detection sample. With GASTON, an inclination, and thus a true mass could be determined for 8 of these companions. For the remaining 21 companions, we could only constrain an upper limit on the true mass, with an astrometric motion compatible with the edge-on inclination within measurement noise. For other 227 candidates, the astrometric excess noise is not large enough to imply a firm detection of the astrometric motion of their host star, but allows us to derive an upper limit on their true mass. They constitute a non-detection sample.

We found that among the detection sample, 30 Ari B b, HD 114762 b, HD 148427 b, HD 5388 b, HD 6718 b, HD 16760 b, and HIP $65891 \mathrm{~b}$ are not planets; rather these candidates are BDs or M-dwarfs. Moreover, we measured a true mass of HD 141937 $\mathrm{b}$, within $9-50 M_{\mathrm{J}}$ compatible at $3 \sigma$ with a planetary nature, although this candidate is a likely BD. 
Among the 227 candidates of the non-detection sample, GASTON applied on the small astrometric excess noise measured by Gaia DR1 confirms that 27 exoplanet candidates are planets. The lower limit on their inclination deduced from the small value of their astrometric excess noise led to an upper limit on mass below $13.5 M_{\mathrm{J}}$ at $3 \sigma$.

These new measurements populate the mass-period diagram in the BD-to-M-dwarf domain, thereby constraining the driest region of the desert of the BD companions detection, also known as the BD desert, to orbital periods smaller than 100 days and mass larger than $20 M_{\mathrm{J}}$. We thus confirm previous estimates of the period threshold of the BD desert, that is, $\sim 100$ days (Ma \& Ge 2014; Ranc et al. 2015; Wilson et al. 2016; Kiefer et al. 2019). Moreover, the distributions of eccentricities and metallicities among BD companions are consistent with a transition from planet-like formation to star-like formation at about $40-50 M_{\mathrm{J}}$ (Ma \& Ge 2014; Maldonado \& Villaver 2017).

Since GASTON allowed us to determine companion masses of few tens of $M_{\mathrm{J}}$ using only preliminary Gaia data products at a precision of $\sim 1$ mas, we can rejoice that future orbital solutions from Gaia astrometric time series at a precision of a few 10-100 $\mu$ as, will allow us to measure the orbital inclination and masses of many RV Jupiter-mass exoplanets and BDs along with new detections among the several billions of sources monitored with Gaia.

Acknowledgements. We thank the anonymous referee for her/his fruitful comments. This work has been funded by the Centre National d'Etudes Spatiales (CNES), and by Paris Science et Lettre (PSL) University.

\section{References}

Akeson, R. L., Chen, X., Ciardi, D., et al. 2013, PASP, 125, 989 Allard, F., Homeier, D., \& Freytag, B. 2012, PTRSLS A, 370, 2765 Anderson, D. R., Hellier, C., Gillon, M., et al. 2010, ApJ, 709, 159 Bakos, G. Á., Hartman, J., Torres, G., et al. 2011, ApJ, 742, 116 Bakos, G. Á., Pál, A., Torres, G., et al. 2009, ApJ, 696, 1950

Baraffe, I., Chabrier, G., Barman, T. S., et al. 2003, A\&A, 402, 701 Bashi, D., Helled, R., Zucker, S., \& Mordasini, C. 2017, A\&A, 604, A83 Bean, J. L., McArthur, B. E., Benedict, G. F., et al. 2007, AJ, 134, 749 Benedict, G. F., McArthur, B. E., Forveille, T., et al. 2002, ApJ, 581, L115 Benedict, G. F., McArthur, B. E., Gatewood, G., et al. 2006, AJ, 132, 2206 Benedict, G. F., McArthur, B. E., Bean, J. L., et al. 2010, AJ, 139, 1844

Bouchy, F., Hébrard, G., Udry, S., et al. 2009, A\&A, 505, 853 Brahm, R., Jones, M., Espinoza, N., et al. 2016, PASP, 128, 124402 Cassan, A., Kubas, D., Beaulieu, J.-P., et al. 2012, Nature, 481, 167 Chabrier, G., Baraffe, I., Allard, F., et al. 2000, ApJ, 542, 464 Christiansen, J. 2018, Handbook of Exoplanets, 150

Courcol, B., Bouchy, F., Pepe, F., et al. 2015, A\&A, 581, A38

Dawson, R. I., Johnson, J. A., Fabrycky, D. C., et al. 2014, ApJ, 791, 89

Demangeon, O. D. S., Faedi, F., Hébrard, G., et al. 2018, A\&A, 610, A63

Demircan, O., \& Kahraman, G. 1991, Ap\&SS, 181, 313

Díaz, R. F., Santerne, A., Sahlmann, J., et al. 2012, A\&A, 538, A113

Díaz, R. F. Damiani, C. Deleuil, M., et al. 2013, A\&A, 551, L9

Duquennoy, A., \& Mayor, M. 1991, A\&A, 500, 337

Faigler, S., Tal-Or, L., Mazeh, T., et al. 2013, ApJ, 771, 26

Fischer, D. A., \& Valenti, J. 2005, ApJ, 622, 1102

Fischer, D., Driscoll, P., Isaacson, H., et al. 2009, ApJ, 703, 1545

Foreman-Mackey, D., Hogg, D. W., Lang, D., et al. 2013, PASP, 125, 306

Gaia Collaboration (Brown, A. G. A., et al.) 2016, A\&A, 595, A2

Gettel, S., Wolszczan, A., Niedzielski, A., et al. 2012, ApJ, 745, 28

Goodman, J., \& Weare, J. 2010, Communications in Applied Mathematics and Computational Science, 5, 65

Guenther, E. W., Hartmann, M., Esposito, M., et al. 2009, A\&A, 507, 1659

Guilluy, G., Sozzetti, A., Brogi, M., et al. 2019, A\&A, 625, A107

Halbwachs, J. L., Arenou, F., Mayor, M., et al. 2000, A\&A, 355, 581

Halbwachs, J. L., Mayor, M., Udry, S., et al. 2003, A\&A, 397, 159
Han, I., Black, D. C., \& Gatewood, G. 2001, ApJ, 548, L57

Han, E., Wang, S. X., Wright, J. T., et al. 2014, PASP, 126, 827

Heacox, W. D. 1995, AJ, 109, 2670

Hébrard, G., Arnold, L., Forveille, T., et al. 2016, A\&A, 588, A145

Hellier, C., Anderson, D. R., Collier Cameron, A., et al. 2011, A\&A, 535, L7

Hellier, C., Anderson, D. R., Collier Cameron, A., et al. 2017, MNRAS, 465, 3693

Hirano, T., Nowak, G., Kuzuhara, M., et al. 2016, ApJ, 825, 53

Ida, S., \& Lin, D. N. C. 2005, ApJ, 626, 1045

Johnson, J. A., Marcy, G. W., Fischer, D. A., et al. 2006, ApJ, 652, 1724

Jones, M. I., Jenkins, J. S., Rojo, P., et al. 2015, A\&A, 580, A14

Jorissen, A., Mayor, M., \& Udry, S. 2001, A\&A, 379, 992

Kane, S. R., Henry, G. W., Dragomir, D., et al. 2011, ApJ, 735, L41

Kane, S. R., Howell, S. B., Horch, E. P., et al. 2014, ApJ, 785, 93

Kervella, P., Arenou, F., \& Schneider, J. 2020, A\&A, 635, L14

Kiefer, F. 2019, A\&A, 632, A9

Kiefer, F., Hébrard, G., Sahlmann, J., et al. 2019, A\&A, 631, A125

Latham, D. W., Mazeh, T., Stefanik, R. P., et al. 1989, Nature, 339, 38

Latyshev, I. N. 1978, Soviet Ast., 22, 186

Lindegren, L., Lammers, U., Hobbs, D., et al. 2012, A\&A, 538, A78

Lindegren, L., Lammers, U., Bastian, U., et al. 2016, A\&A, 595, A4

Lindegren, L., Hernández, J., Bombrun, A., et al. 2018, A\&A, 616, A2

Lovis, C., \& Fischer, D. 2010, Exoplanets, 27

Lucy, L. B. 1974, AJ, 79, 745

Ma, B., \& Ge, J. 2014, MNRAS, 439, 2781

Maldonado, J., \& Villaver, E. 2017, A\&A, 602, A38

Malkov, O. Y. 2007, MNRAS, 382, 1073

Marcy, G. W., \& Butler, R. P. 2000, PASP, 112, 137

Marcy, G. W., Isaacson, H., Howard, A. W., et al. 2014, ApJS, 210, 20

Martioli, E., McArthur, B. E., Benedict, G. F., et al. 2010, ApJ, 708, 625

Mayor, M., Marmier, M., Lovis, C., et al. 2011 ArXiv e-prints [arXiv:1109.2497]

Mazeh, T., \& Goldberg, D. 1992, ApJ, 394, 592

Mazeh, T., Zucker, S., Dalla Torre, A., et al. 1999, ApJ, 522, L149

Mazeh, T., Holczer, T., \& Faigler, S. 2016, A\&A, 589, A75

McArthur, B. E., Benedict, G. F., Henry, G. W., et al. 2014, ApJ, 795, 41

McCarthy, C., Butler, R. P., Tinney, C. G., et al. 2004, ApJ, 617, 575

Michalik, D., Lindegren, L., \& Hobbs, D. 2015a, A\&A, 574, A115

Michalik, D., Lindegren, L., Hobbs, D., et al. 2015b, A\&A, 583, A68

Močnik, T., Anderson, D. R., Brown, D. J. A., et al. 2016, PASP, 128, 124403

Moutou, C., Loeillet, B., Bouchy, F., et al. 2006, A\&A, 458, 327

Naef, D., Mayor, M., Lo Curto, G., et al. 2010, A\&A, 523, A15

Osborn, H. P., Santerne, A., Barros, S. C. C., et al. 2017, A\&A, 604, A19

O’Toole, S. J., Butler, R. P., Tinney, C. G., et al. 2007, ApJ, 660, 1636

Perruchot, S., Bouchy, F., Chazelas, B., et al. 2011, Proc. SPIE, 815115

Perryman, M. A. C., Lindegren, L., Arenou, F., et al. 1996, A\&A, 310, L21

Perryman, M., Hartman, J., Bakos, G. Á., et al. 2014, ApJ, 797, 14

Pourbaix, D., Tokovinin, A. A., Batten, A. H., et al. 2004, A\&A, 424, 727

Pollack, J. B., Hubickyj, O., Bodenheimer, P., et al. 1996, Icarus, 124, 62

Raghavan, D., McAlister, H. A., Henry, T. J., et al. 2010, ApJS, 190, 1

Ranc, C., Cassan, A., Albrow, M. D., et al. 2015, A\&A, 580, A125

Rasio, F. A., \& Ford, E. B. 1996, Science, 274, 954

Reffert, S., \& Quirrenbach, A. 2011, A\&A, 527, A140

Richardson, W. H. 1972, JOSA 1917-1983, 62, 55

Sahlmann, J., Ségransan, D., Queloz, D., et al. 2011a, A\&A, 525, A95

Sahlmann, J., Lovis, C., Queloz, D., et al. 2011b, A\&A, 528, L8

Santos, N. C., Mayor, M., Benz, W., et al. 2010, A\&A, 512, A47

Sato, B., Fischer, D. A., Ida, S., et al. 2009, ApJ, 703, 671

Schneider, J., Dedieu, C., Le Sidaner, P., et al. 2011, A\&A, 532, A79

Shahaf, S., Mazeh, T., \& Faigler, S. 2017, MNRAS, 472, 4497

Simpson, E. K., Baliunas, S. L., Henry, G. W., et al. 2010, MNRAS, 408, 1666

Sozzetti, A., \& Desidera, S. 2010, A\&A, 509, A103

Tabachnik, S., \& Tremaine, S. 2002, MNRAS, 335, 151

Torres, G., Andersen, J., \& Giménez, A. 2010, A\&ARv, 18, 67

Triaud, A. H. M. J., Collier Cameron, A., Queloz, D., et al. 2010, A\&A, 524, A25

Udry, S., Mayor, M., Naef, D., et al. 2002, A\&A, 390, 267

van de Kamp, P. 1975, ARA\&A, 13, 295

West, R. G., Collier Cameron, A., Hebb, L., et al. 2009, A\&A, 502, 395

Wilson, P. A., Hébrard, G., Santos, N. C., et al. 2016, A\&A, 588, A144

Wittenmyer, R. A., Horner, J., Tinney, C. G., et al. 2014, ApJ, 783, 103

Wright, J. T., Fakhouri, O., Marcy, G. W., et al. 2011, PASP, 123, 412

Zucker, S., \& Mazeh, T. 2001, ApJ, 562, 549

Zucker, S., \& Mazeh, T. 2001, ApJ, 562, 1038 


\section{Appendix A: Magnitude of the companion}

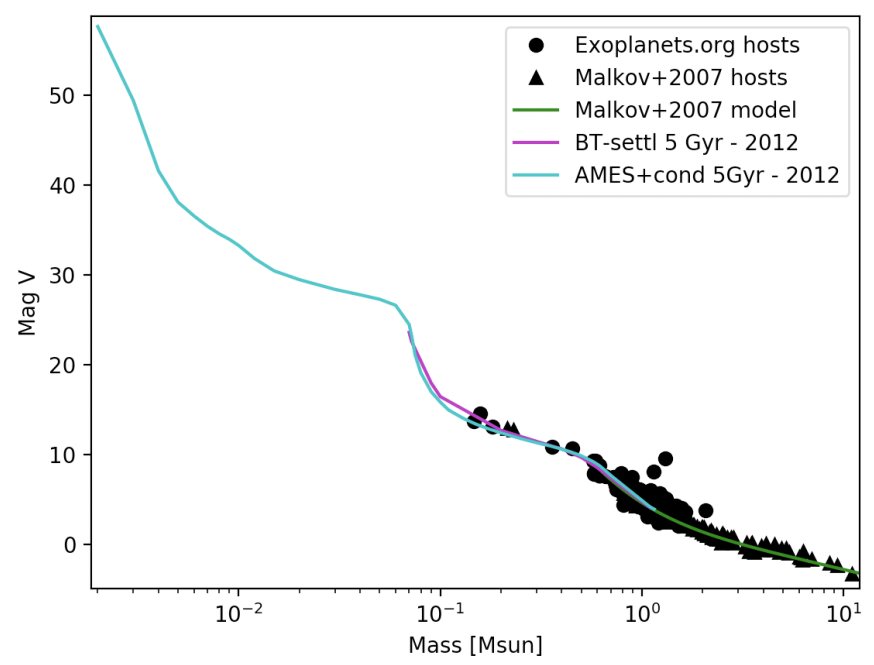

Fig. A.1. Absolute visual magnitude of emitted light against mass from low-mass BD up to massive stars. Data are taken from the exoplanets.org catalog and Malkov (2007). Solid lines show the models, as presented in the legend.

To calculate the impact of the light emitted from the companion on the apparent primary semimajor axis, as observed by Gaia (also known as the photocenter semimajor axis) we have to take two effects into account: the emission from the companion (c) itself, if not planetary, and the star $(\star)$ light reflected by the companion toward the observer. We recall the equation of the photocenter semimajor axis given in Kiefer et al. (2019) (see also van de Kamp 1975 for the original calculation) as follows:

$a_{\text {phot }}=(B-\beta) a_{\text {tot }} \quad$ with $a_{\text {tot }}=a_{\mathrm{c}}+a_{\star}$.

We introduced the luminosity fraction $\beta=L_{\mathrm{c}} /\left(L_{\mathrm{c}}+L_{\star}\right)$ and the mass fraction $B=q /(1+q)$ with $q=M_{\mathrm{c}} / M_{\star}$ the mass ratio. The key parameter is the luminosity fraction.

In order to calculate the emitted $V$ magnitude of the components, we use multiple empirical models. We use the given $V$ magnitude as an approximation of the $G$ magnitude. Since only the $\delta G$ is important for our study, we do not expect strong deviations resulting from this approximation. These models are listed below and presented in Fig. A.1, compared to published data from exoplanets.org and Malkov (2007) in the Johnson $V$ band, as follows:

- Allard et al. (2012) AMES-cond isochrone at $5 \mathrm{Gyr}$, for the BD-to-dM regime;

- BT-Settl (Allard et al. 2012, and references therein) isochrone at $5 \mathrm{Gyr}$, for the stellar regime up to $1.1 M_{\odot}$;

- Malkov (2007) empirical model above $1.1 M_{\odot}$ up to $10 M_{\odot}$. Only visual magnitudes for objects with mass larger than $0.1 M_{\odot}$ could be found in the literature, owing to the difficulty for observing faint objects such as BDs and exoplanets in the optical band. Therefore, we can only assess the validity of the $V$-mag models in the stellar domain and we assume their validity in the $G$ band down to the planetary domain owing moreover to the relative compatibility of the AMES-cond models with observations in the infrared (Chabrier et al. 2000; Baraffe et al. 2003; Allard et al. 2012). The BT-settl model seems more accurate than AMES-cond in the IR for massive BDs (Allard et al. 2012), but it does not give magnitudes for objects with mass below $0.75 M_{\odot}$. In order to insure continuity of the mass-magnitude relation we

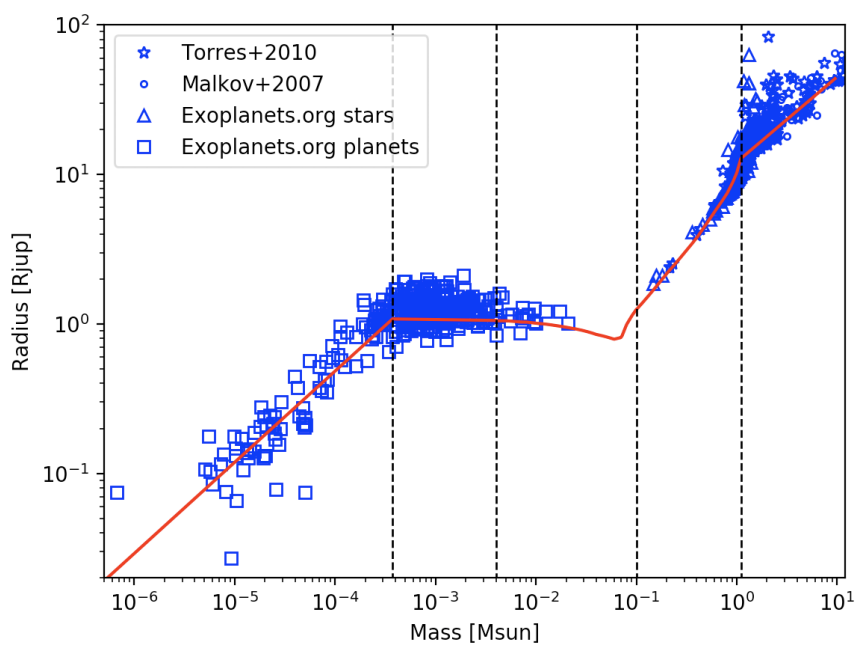

Fig. A.2. Radius against mass from terrestrial mass planets up to massive stars. Data are taken from the exoplanets.org catalog, Malkov (2007), and Torres et al. (2010). The red solid line represents the continuous model, comprised of Bashi et al. (2017) from $10^{-6}$ to $0.004 M_{\odot}$, Baraffe et al. (2003) from 0.004 to $0.1 M_{\odot}$, BT-settl from 0.1 to $1.1 M_{\odot}$, and the empirical relation $R \propto M^{0.57}$ above $1.1 M_{\odot}$ (e.g., Demircan \& Kahraman 1991).

prefer to use the AMES-cond model from masses of $0.1 M_{\odot}$ down to $10 M_{\mathrm{J}}$.

For the reflected light, we calculate the mean reflection along an entire orbit. For the whole domain from planets to stars, we assume the body is a Lambertian sphere with a typical Bond albedo of 0.3 . The radius of the sphere is related to the mass of the body. There is no continuous exact law $R=f(M)$ on the whole planet-to-star domain, with a wide diversity of densities for planets and also for stars if we account for (sub-)giants stars. However, for the sake of continuity and simplicity, we assume a common continuous law relating the radius of a body to its mass. This avoids issues concerning gaps in the MCMC and allows us to derive well-behaved posterior distributions. This law is established using several segments and is presented in Fig. A.2. The data are taken from several sources as follows:

- Low-mass planets up to $0.5 M_{\mathrm{J}}$, with the empirical relations of Bashi et al. (2017);

- Jovian-mass planets and BDs up to $0.1 M_{\odot}$ stars along an AMES-cond isochrone at 5 Gyr (Allard et al. 2012);

- Low-mass stars, from 0.1 to $1.1 M_{\odot}$, with the BT-settl model (Allard et al. 2012, and references therein) along an isochrone at $5 \mathrm{Gyr}$,

- More massive stars up to $10 M_{\odot}$, with the empirical $R \propto$ $M^{0.57}$ relationship (e.g., Demircan \& Kahraman 1991; Torres et al. 2010).

Taking into account emission and reflection from the companion, we can calculate magnitude differences between the primary and the companion for different values of their mass and of the semimajor axis of their orbit. This is plotted in Fig. A.3. The net impact on the apparent primary semimajor axis can be measured by comparing the photocenter semimajor axis to the primary semimajor axis. This is presented in Fig. A.4. In general, the semimajor axis of the photocenter orbit decreases with increasing contribution of the companion in the total luminosity of the system.

In the stellar regime, the impact of the companion starts to be significant on the apparent primary semimajor axis for companion masses larger than about $20 \%$ of the primary star mass. For 


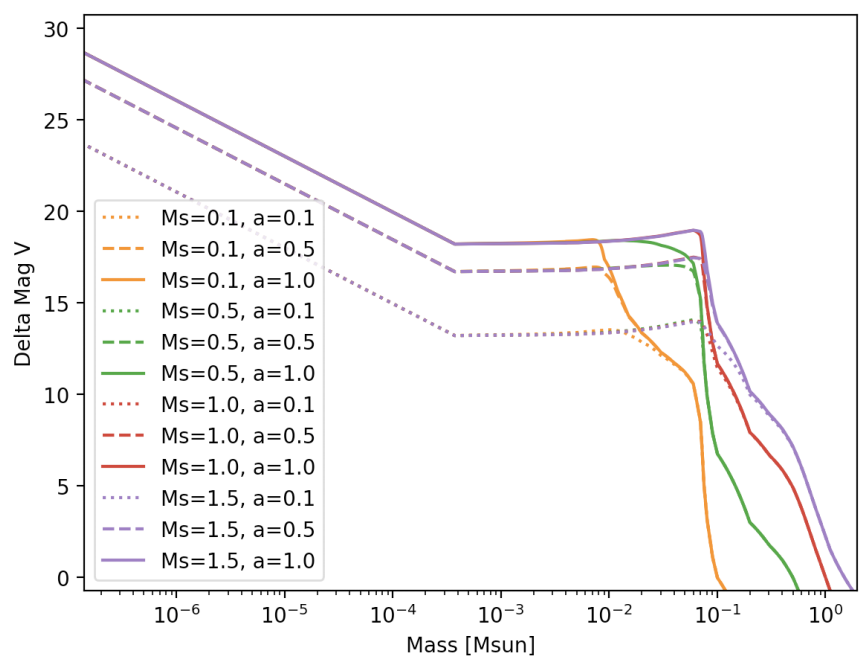

Fig. A.3. Adopted continuous model of $\Delta V$ with respect to the mass ratio. On this plot, the primary is assumed with a mass ranging from 0.1 to $1.5 M_{\odot}$, and the secondary is a Lambertian sphere with average Bond albedo of 0.3 on an orbit with semimajor axis between 0.1 au and 1 au.

a primary of mass $0.5 M_{\odot}$ the magnitude of the secondary has a measurable effect for a mass larger than about $0.1 M_{\odot}$. If the secondary is too luminous compared to the primary, the semimajor axis of the photocenter can even reach 0 . However, this effect remains hidden in the present study because we impose that the companion is dark with $\Delta V>2.5$.

In the planetary mass regime, on the other hand, the impact of the companion on the photocenter can be strong if the semimajor axis of the companion orbit is smaller than 0.5 au and the mass ratio $q=M_{\mathrm{c}} / M_{\star} \sim 10^{-5}-10^{-3}$. The impact is stronger for earlier primaries. This is due to the shortening of the primary star orbit with decreasing companion mass, while the companion radius is relatively constant about $1 R_{J}$ down to a few fractions of Jupiter mass (Bashi et al. 2017). In this regime, the astrometric motion of the system observed from Earth, although of small extent, can actually follow the motion of the companion rather than that of the stellar host. This happens precisely when the luminosity ratio $L_{\mathrm{c}} / L_{\star}>q$.

Nevertheless, this effect does not have a strong role in the present study because $q \sim 10^{-5}-10^{-3}$ with $a_{\mathrm{c}}<0.5$ au implies $a_{\star} \ll a_{\mathrm{c}}$ and $a_{\mathrm{ph}}<10^{-3}$ au. In the worst case scenario, it could only lead at most to an astrometric motion of $\sim 0.5$ mas if the parallax is $\sim 500$ mas. This is well below the detection thresholds $\left(\varepsilon_{\text {thresh,prim }}=0.85\right.$ mas and $\varepsilon_{\text {thresh,second }}=1.2$ mas $)$ defined
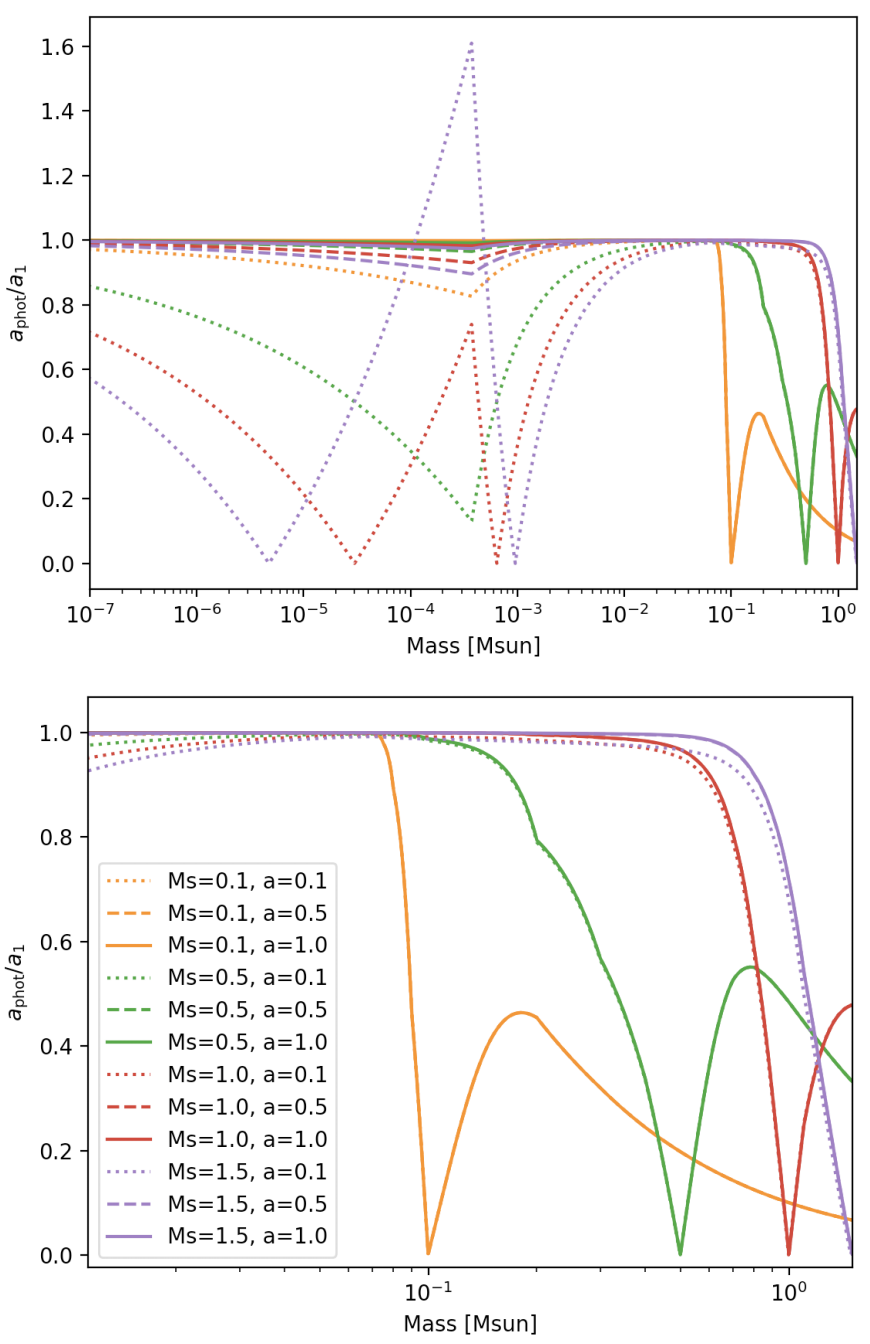

Fig. A.4. Effect of emitted and reflected light by the secondary companion on the photocenter semimajor axis. Top: full mass ratio range. Bottom: beyond $M=0.1 M_{\odot}$. Captions are identical in the two panels.

in Sect. 3.5 and this situation is thus undetectable within the diverse noises accounted for in Gaia measurements. However, this will be an important effect to account for in future analysis of Gaia time series when this precision is reached. Neglecting it might lead to strongly underestimating the star semimajor axis and, thus, the mass of the companion. 